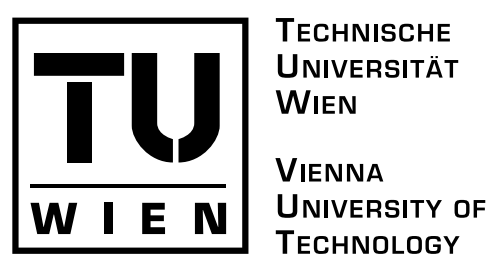

M.Sc. ARBEIT

\title{
Herbrand Sequent Extraction
}

\author{
ausgeführt am \\ Institut für Computersprachen \\ Arbeitsgruppe Theoretische Informatik und Logik \\ der Technischen Universität Wien \\ unter der Anleitung von \\ Univ.Prof. Dr.phil. Alexander Leitsch \\ durch \\ Bruno Woltzenlogel Paleo
}

Wien, 04. Mai 2007

Bruno Woltzenlogel Paleo 
Alexander Leitsch

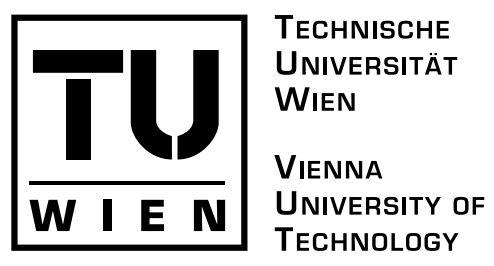

\title{
Master Thesis
}

\section{Herbrand Sequent Extraction}

\author{
carried out at the \\ Institute of Computer Languages \\ Theory and Logic Group \\ of the Vienna University of Technology \\ under the instruction of \\ Univ.Prof. Dr.phil. Alexander Leitsch \\ by \\ Bruno Woltzenlogel Paleo
}

Vienna, May 04, 2007

Bruno Woltzenlogel Paleo 


\section{Contents}

Kurzfassung $\quad$ v

Abstract vii

Dedication $\quad$ ix

Acknowledgements $\quad$ xi

1 Introduction 1

2 Notations and Definitions 3

2.1 Meta-Notations . . . . . . . . . . . . . . . . 3

2.2 First-Order Logic . . . . . . . . . . . . . . . . . . . 3

2.2 .1 The Language . . . . . . . . . . . . . . . . 4

2.2.2 Manipulation of Formulas . . . . . . . . . . . 5

2.2.3 Classifications . . . . . . . . . . . . 7

2.3 Sequent Calculus . . . . . . . . . . . . . . 8

2.3 .1 Sequents . . . . . . . . . . . . . . . 9

2.3 .2 Inference Rules . . . . . . . . . . . . . . . . . . 11

2.3 .3 LK-Proofs . . . . . . . . . . . . . . . . . 13

2.3.4 Positions and Substitutions in Proofs . . . . . . . . . 14

2.3.5 Formula Occurrences in Proofs . . . . . . . . . . . 16

2.4 Summary of Notations for Positions and Substitutions . . . . 17

3 Proof Analysis and Proof Transformations 19

3.1 Prenex Form . . . . . . . . . . . . . . . . . . 19

3.2 Skolemization . . . . . . . . . . . . . . . 20

3.3 Herbrand Sequent . . . . . . . . . . . . . . . . 22

3.4 Cut-Elimination . . . . . . . . . . . . . 23

4 Extraction of Herbrand Sequents $\quad 25$

4.1 Extraction from Prenex Proofs . . . . . . . . . . . . 27

4.1.1 Extraction via Mid-Sequent Reduction . . . . . . . . 28

4.1.2 Extraction via Collection of Instances . . . . . . . . 42 
4.2 Extraction from Non-Prenex Proofs . . . . . . . . . 47 4.2.1 Extraction via Proof Transformation to Quantifierfree $\mathbf{L K}_{\mathbf{A}} \ldots \ldots \ldots \ldots \ldots$

4.2.2 Extraction via Collection of Sub-Formula Instances . . 55

5 Conclusion 


\section{Kurzfassung}

Nach der Definition einer Verallgemeinerung des Herbrandschen Theorems für Sequente, beschreiben wir drei schon existierende Algorithmen für die Extraktion eines Herbrandsequents aus formalen Beweisen, die im Sequentialkalkül LK geschrieben sind. Darüber hinaus verbessern wir diese drei Algorithmen und entwicklen daraus einen vierten Algorithmus, welcher deren Ideen vereinigt und generalisiert. Dieser Algorithmus wurde dann realisiert und in das CERes (Cut-Elimination by Resolution) Projekt integriert. Die Wichtigkeit der Extraktion von Herbrandsequenten liegt darin, dass Herbrandsequente die Kreativität der Beweise enthält. 


\begin{abstract}
After defining a generalization of Herbrand's Theorem for sequents, we describe three pre-existing algorithms for the extraction of a Herbrand sequent of the end-sequent of proofs in the Sequent Calculus LK. Furthermore, we improve these three algorithms by designing a new fourth algorithm, which combines and generalizes their essential ideas. The implementation of this new algorithm was realized within the framework of the project CERes (Cut-Elimination by Resolution). The importance of extracting Herbrand sequents from proofs lies on the fact that a Herbrand sequent summarizes the creative content of a proof.
\end{abstract}




\section{Dedication}

I dedicate this master thesis to my parents, who in spite of the distance, continued to support me with their love and attention. 


\section{Acknowledgements}

I would like to thank my supervisor, Prof. Alexander Leitsch, for providing an interesting, relevant and useful topic for this Master Thesis, and for advising me, pointing me the good directions to pursue in moments of doubts, and revising this thesis.

The discussions with Prof. Leitsch and his Ph.D. students in the weekly meetings of the CERes Project were also very influential to this thesis. They not only gave me knowledge and a broader view on the area in which this work is inserted, but they also transmitted their motivation to me. Specially, I would like to thank Stefan Hetzl, for very interesting discussions about my theoretical doubts, and both Clemens Richter and Stefan Hetzl, for explaining the source code of CERes.

My studies at TU-Dresden and TU-Wien were supported by the Programme $\mathrm{Al} \beta \mathrm{an}$, the European Union Programme of High Level Scholarships for Latin America (scholarship no. E05M054053BR).

I am also grateful to colleagues and friends who shared time, experiences, fun and knowledge with me during this period of one year in Germany and one year in Austria. 


\section{Chapter 1}

\section{Introduction}

In Proof Theory, we are interested in constructing and analyzing formal proofs, which strictly follow axioms and rules of inference of formal logical proof calculi, as Hilbert Calculi, Natural Deduction Calculi or Sequent Calculi. Some of the main advantages of the formalization of proofs are:

- The correctness of formal proofs can be easily checked, by verifying whether the formal axioms and rules of the calculus were correctly employed.

- Formal proofs for formalized statements (formulas) may be constructed by computers executing Automatic Theorem Provers, as in [11].

- New informal proofs for statements may be discovered by applying formal transformations to their corresponding formal proofs, as in [3] and $[4]$.

- Formal proofs, when viewed and studied as a model and ideal for informal mathematical proofs, allow meta-mathematical investigations into the foundations of Mathematics.

However, formal representations of real informal mathematical proofs or computer generated proofs of real mathematical problems usually have some drawbacks that make them difficult to be analyzed and understood by mathematicians or by humans in general. Firstly, the size of a formal proof is usually huge ([2]), which makes it hard to be visualized in its whole. Secondly, many of its individual inferences are only structural, necessary not to carry some essential idea about the proof, but only to satisfy the formalities of the calculus used. Thirdly, inference rules of proof calculi not always correspond easily to natural inferences in informal proofs. Together these drawbacks imply that, given a formal proof, it is not easy for a human to extract its essential idea, because one gets lost in a proof which looks like a huge data structure of repetitive, bureaucratic and non-intuitive formalities 
that involuntarily hide the crucial information. Therefore there is a clear need for summarization of formal proofs or for extraction of its essential ideas, whenever these proofs are intended to be analyzed and understood by humans.

This thesis describes one possible technique that helps to fulfill this need in the particular case of First-Order Logic and Sequent Calculus LK. Our technique relies on the concept of Herbrand Sequent (a generalization of Herbrand Disjunction ([9]) and its extraction from LK-Proofs in a way that summarizes their creative content, which lies on the instantiations chosen for quantified variables.

The following chapters form this thesis:

- Chapter 2, Notations and Definitions, defines the language used for First-Order Logic and the variant of Sequent Calculus LK used.

- Chapter 3, Proof Analysis and Transformations, defines the concept of Herbrand sequent and describes important and related results, as Herbrand's Theorem and Gentzen's Mid-Sequent Theorem, which are essential to the methods described in the next chapter.

- Chapter 4, Extraction of Herbrand Sequents, has four subsections, each devoted to a different algorithm for the extraction of Herbrand Sequents.

The novel contribution made by this thesis is the fourth algorithm, $E x$ traction via Collection of Sub-Formula Instances, which combines and generalizes the three pre-existing algorithms. 


\section{Chapter 2}

\section{Notations and Definitions}

The notations and definitions described in this chapter construct the basic formal language and the formal proof calculus which are used in this thesis: First-Order Logic and the Sequent Calculus LK. Together they allow the formalization of proofs.

\section{$2.1 \quad$ Meta-Notations}

The symbol $(=)$ is usually used ambiguously in science. Here we reduce this ambiguity by using the two different symbols below:

- $\doteq$ : is used to define a simpler notation for a, usually more complex, existing expression. Thus $A \doteq B$ implies that we may use $A$ as a shorthand notation for $B$.

- =: is used to assert that two expressions are syntactically equal.

\section{$2.2 \quad$ First-Order Logic}

In this section we firstly describe a language for First-Order Logic, which consists of an alphabet and formation rules to construct well-formed terms and formulas from these.

Secondly, notations for the manipulation of formulas are described. These notations allow reference to sub-formulas in specific positions of the formula and substitution of variables or sub-formulas in formulas.

And finally, further definitions are given, which distinguish and classify special properties owned by certain sub-formulas, quantifiers and variables. These classifications are important for the definition of the algorithms that will be described in the subsequent chapters. 


\subsubsection{The Language}

Definition 2.2.1 (Alphabet of First-Order Logic). The Alphabet of FirstOrder Logic consists of:

1. A countable set of variables $V$

2. A countable set of constant symbols $C$

3. For every $n \geq 1$, a countable set of function symbols $F_{n}$. $\left(F \doteq \bigcup_{n \geq 1} F_{n}\right.$ is the set of all function symbols).

4. For every $n \geq 1$, a countable set of predicate symbols $P_{n}$. $(P \doteq$ $\bigcup_{n \geq 1} P_{n}$ is the set of all predicate symbols).

5. A set of propositional connectives $\{\vee, \wedge, \rightarrow, \neg\}$

6. A set of quantifiers $\{\forall, \exists\}$

7. The set of parentheses $\{()$,

8. The set of symbols $\{\top, \perp\}$

Definition 2.2.2 (Terms). The set of terms $T$ is defined as the smallest set satisfying:

1. $V \subset T$

2. $C \subset T$

3. For all $n \geq 1$ : If $f \in F_{n}$ and $t_{1}, \ldots, t_{n} \in T$, then $f\left(t_{1}, \ldots, t_{n}\right) \in T$.

Definition 2.2.3 (Formulas). The set of formulas $F_{F O L}$ is defined as the smallest set satisfying:

1. For all $n \geq 1$ : If $P \in P_{n}$ and $t_{1}, \ldots, t_{n} \in T$, then $P\left(t_{1}, \ldots, t_{n}\right) \in F_{F O L}$.

2. If $A \in F_{F O L}$, then $\neg A \in F_{F O L}$

3. If $A, B \in F_{F O L}$, then $(A \wedge B) \in F_{F O L}$

4. If $A, B \in F_{F O L}$, then $(A \vee B) \in F_{F O L}$

5. If $A, B \in F_{F O L}$, then $(A \rightarrow B) \in F_{F O L}$

6. If $A \in F_{F O L}$ and $x \in V$, then $(\forall x) A \in F_{F O L}$

7. If $A \in F_{F O L}$ and $x \in V$, then $(\exists x) A \in F_{F O L}$

For the sake of transparency, we usually omit the outermost parentheses of a formula. 


\subsubsection{Manipulation of Formulas}

Definition 2.2.4 (Positions of a Formula). Let $A$ be a formula, then its set of positions $\operatorname{pos}(A)$ is the smallest set of strings belonging to $\{1,2\}^{*}$ and such that $\operatorname{pos}(A)$ satisfies:

1. $\varepsilon \in \operatorname{pos}(A)$

2. If $A$ is of the form $\neg B$, then $\{1 . \lambda \mid \lambda \in \operatorname{pos}(B)\} \subset \operatorname{pos}(A)$.

3. If $A$ is of the form $(B \wedge C)$, then $\left(\left\{1 . \lambda_{1} \mid \lambda_{1} \in \operatorname{pos}(B)\right\} \cup\left\{2 . \lambda_{2} \mid \lambda_{2} \in\right.\right.$ $\operatorname{pos}(C)\}) \subset \operatorname{pos}(A)$.

4. If $A$ is of the form $(B \vee C)$, then $\left(\left\{1 . \lambda_{1} \mid \lambda_{1} \in \operatorname{pos}(B)\right\} \cup\left\{2 . \lambda_{2} \mid \lambda_{2} \in\right.\right.$ $\operatorname{pos}(C)\}) \subset \operatorname{pos}(A)$.

5. If $A$ is of the form $(B \rightarrow C)$, then $\left(\left\{1 . \lambda_{1} \mid \lambda_{1} \in \operatorname{pos}(B)\right\} \cup\left\{2 . \lambda_{2} \mid \lambda_{2} \in\right.\right.$ $\operatorname{pos}(C)\}) \subset \operatorname{pos}(A)$.

6. If $A$ is of the form $(\forall x) B$, then $\{1 . \lambda \mid \lambda \in \operatorname{pos}(B)\} \subset \operatorname{pos}(A)$.

7. If $A$ is of the form $(\exists x) B$, then $\{1 . \lambda \mid \lambda \in \operatorname{pos}(B)\} \subset \operatorname{pos}(A)$.

Example 2.1 (Positions of a Formula). Below are the sets of positions for some example formulas:

1. $\operatorname{pos}(F(a) \vee G(b))=\{\varepsilon, 1,2\}$

2. $\operatorname{pos}(F(a) \vee(G(b) \wedge F(b)))=\{\varepsilon, 1,2,21,22\}$

3. $\operatorname{pos}((\forall x) F(x) \vee(G(b) \wedge F(b)))=\{\varepsilon, 1,2,11,21,22\}$

4. $\operatorname{pos}(F(c) \vee(\exists x)(G(x) \wedge F(b)))=\{\varepsilon, 1,2,21,211,212\}$

Definition 2.2.5 (Depth of a Position of a Formula). Let $\lambda$ be a position of a formula $F$. Then, the depth of $\lambda$, depth $(\lambda)$ is defined inductively:

1. $\operatorname{depth}(\epsilon) \doteq 0$.

2. $\operatorname{depth}\left(1 . \lambda^{\prime}\right) \doteq 1+\operatorname{depth}\left(\lambda^{\prime}\right)$.

3. $\operatorname{depth}\left(2 . \lambda^{\prime}\right) \doteq 1+\operatorname{depth}\left(\lambda^{\prime}\right)$.

Definition 2.2.6 (Atomic Formulas, Compound Formulas). A formula $A$ is atomic if $\operatorname{pos}(A)=\{\varepsilon\}$. In this case, $A$ is clearly of the form $P\left(t_{1}, \ldots, t_{n}\right)$, where $P \in P_{n}$ and $t_{1}, \ldots, t_{n} \in T$. Otherwise, it is said to be compound.

Definition 2.2.7 (Sub-formulas occurring in given Positions). Let $\lambda$ be a position of the formula $A$. Then the sub-formula occurring in position $\lambda$ is denoted $|A|_{\lambda}$. Formally, we may define the computation of $|A|_{\lambda}$ inductively: 
1. $|A|_{\varepsilon} \doteq A$

2. If $A$ is of the form $\neg B$ and $\lambda=1 . \lambda^{\prime}$ with $\lambda^{\prime} \in \operatorname{pos}(B)$, then $|A|_{\lambda} \doteq$ $|B|_{\lambda^{\prime}}$.

3. If $A$ is of the form $B \circ C$ (for $\circ \in\{\wedge, \vee, \rightarrow\}$ ) and $\lambda=1 . \lambda^{\prime}$ with $\lambda^{\prime} \in \operatorname{pos}(B)$, then $|A|_{\lambda} \doteq|B|_{\lambda^{\prime}}$.

4. If $A$ is of the form $B \circ C$ (for $\circ \in\{\wedge, \vee, \rightarrow\}$ ) and $\lambda=2 . \lambda^{\prime}$ with $\lambda^{\prime} \in \operatorname{pos}(C)$, then $|A|_{\lambda} \doteq|C|_{\lambda^{\prime}}$.

5. If $A$ is of the form $(Q x) B$ (for $Q \in\{\forall, \exists\}$ ) and $\lambda=1 . \lambda^{\prime}$ with $\lambda^{\prime} \in$ $\operatorname{pos}(B)$, then $|A|_{\lambda} \doteq|B|_{\lambda^{\prime}}$.

Example 2.2 (Sub-formulas occurring in given Positions). Below are some examples of sub-formulas occurring in specified positions of the formula $F(c) \vee(\exists x)(G(x) \wedge F(b))$ :

1. $|F(c) \vee(\exists x)(G(x) \wedge F(b))|_{\varepsilon}=F(c) \vee(\exists x)(G(x) \wedge F(b))$

2. $|F(c) \vee(\exists x)(G(x) \wedge F(b))|_{1}=F(c)$

3. $|F(c) \vee(\exists x)(G(x) \wedge F(b))|_{2}=(\exists x)(G(x) \wedge F(b))$

4. $|F(c) \vee(\exists x)(G(x) \wedge F(b))|_{21}=(G(x) \wedge F(b))$

5. $|F(c) \vee(\exists x)(G(x) \wedge F(b))|_{211}=G(x)$

6. $|F(c) \vee(\exists x)(G(x) \wedge F(b))|_{212}=F(b)$

Definition 2.2.8 (Sub-formula Substitution). Let $\lambda$ be a position of the formula $A$ such that $B \doteq|A|_{\lambda}$. The formula obtained by substituting the occurrence of $B$ in position $\lambda$ in $A$ by $C$ is denoted $A_{\lambda}[C]$ or $A \sigma^{f o}$, where $\sigma^{f o} \doteq\{\lambda / C\}$

Example 2.3 (Sub-formula Substitutions in a Formula). Below are some examples of substitutions of sub-formulas occurring in specified positions of the formula $F(c) \vee(\exists x)(G(x) \wedge F(b))$ by the formula $S(s)$ :

1. $(F(c) \vee(\exists x)(G(x) \wedge F(b)))_{\varepsilon}[S(s)]=S(s)$

2. $(F(c) \vee(\exists x)(G(x) \wedge F(b)))_{1}[S(s)]=S(s) \vee(\exists x)(G(x) \wedge F(b))$

3. $(F(c) \vee(\exists x)(G(x) \wedge F(b)))_{2}[S(s)]=F(c) \vee S(s)$

4. $(F(c) \vee(\exists x)(G(x) \wedge F(b)))_{21}[S(s)]=F(c) \vee(\exists x) S(s)$

5. $(F(c) \vee(\exists x)(G(x) \wedge F(b)))_{211}[S(s)]=F(c) \vee(\exists x)(S(s) \wedge F(b))$

6. $(F(c) \vee(\exists x)(G(x) \wedge F(b)))_{212}[S(s)]=F(c) \vee(\exists x)(G(x) \wedge S(s))$ 


\subsubsection{Classifications}

Definition 2.2.9 (Positive and Negative Sub-formulas). Let $B$ be a subformula of a First-Order Logic formula $A$. We classify $B$ as a positive or negative Sub-formula of $A$ according to the following cases:

- If $B=A$, then $B$ is a positive sub-formula of $A$.

- If $A=\neg A_{1}$ and $B$ is a positive sub-formula of $A_{1}$, then $B$ is a negative sub-formula of $A$.

- If $A=\neg A_{1}$ and $B$ is a negative sub-formula of $A_{1}$, then $B$ is a positive sub-formula of $A$.

- If $A=\left(A_{1} \vee A_{2}\right)$ and $B$ is a positive sub-formula of $A_{1}$, then $B$ is a positive sub-formula of $A$.

- If $A=\left(A_{1} \vee A_{2}\right)$ and $B$ is a positive sub-formula of $A_{2}$, then $B$ is a positive sub-formula of $A$.

- If $A=\left(A_{1} \vee A_{2}\right)$ and $B$ is a negative sub-formula of $A_{1}$, then $B$ is a negative sub-formula of $A$.

- If $A=\left(A_{1} \vee A_{2}\right)$ and $B$ is a negative sub-formula of $A_{2}$, then $B$ is a negative sub-formula of $A$.

- If $A=\left(A_{1} \wedge A_{2}\right)$ and $B$ is a positive sub-formula of $A_{1}$, then $B$ is a positive sub-formula of $A$.

- If $A=\left(A_{1} \wedge A_{2}\right)$ and $B$ is a positive sub-formula of $A_{2}$, then $B$ is a positive sub-formula of $A$.

- If $A=\left(A_{1} \wedge A_{2}\right)$ and $B$ is a negative sub-formula of $A_{1}$, then $B$ is a negative sub-formula of $A$.

- If $A=\left(A_{1} \wedge A_{2}\right)$ and $B$ is a negative sub-formula of $A_{2}$, then $B$ is a negative sub-formula of $A$.

- If $A=\left(A_{1} \rightarrow A_{2}\right)$ and $B$ is a positive sub-formula of $A_{1}$, then $B$ is a negative sub-formula of $A$.

- If $A=\left(A_{1} \rightarrow A_{2}\right)$ and $B$ is a positive sub-formula of $A_{2}$, then $B$ is a positive sub-formula of $A$.

- If $A=\left(A_{1} \rightarrow A_{2}\right)$ and $B$ is a negative sub-formula of $A_{1}$, then $B$ is a positive sub-formula of $A$.

- If $A=\left(A_{1} \rightarrow A_{2}\right)$ and $B$ is a negative sub-formula of $A_{2}$, then $B$ is a negative sub-formula of $A$. 
Definition 2.2.10 (Strong and Weak Quantifiers). Let $B \doteq(Q x) B^{\prime}$ be a sub-formula of $A$. We classify $Q$ as strong or weak according to the following cases:

- If $Q=\forall$ and $B$ is a positive sub-formula of $A$, then $Q$ is strong in $A$.

- If $Q=\forall$ and $B$ is a negative sub-formula of $A$, then $Q$ is weak in $A$.

- If $Q=\exists$ and $B$ is a positive sub-formula of $A$, then $Q$ is weak in $A$.

- If $Q=\exists$ and $B$ is a negative sub-formula of $A$, then $Q$ is strong in $A$.

Definition 2.2.11 (Scope of Quantifiers, Bound and Free Variables). Let $A$ be a formula of the form $(Q x) B$, with $Q \in\{\forall, \exists\}$ and $B \in F_{F O L} . B$ and all its sub-formulas and variables are said to be in the scope of $(Q x)$. A variable $x$ is said to be bound if it is in the scope of $(Q x)$. A variable $x$ is said to be free if it is not in the scope of any $(Q x)$.

Definition 2.2.12 (Variable Substitution). $\sigma \doteq\left\{v_{1} \leftarrow t_{1}, \ldots, v_{n} \leftarrow t_{n}\right\}$ denotes a variable substitution. $A \sigma$ is the formula obtained from $A$ by substituting all occurrences of free-variables $v_{j}$ by the corresponding terms $t_{j}$.

Definition 2.2.13 (Unifier). Let $A$ and $B$ be formulas. If there is a variable substitution $\sigma$ such that $A \sigma=B \sigma$, then $A$ and $B$ are said to be unifiable and $\sigma$ is called a unifier for $A$ and $B$.

\subsection{Sequent Calculus}

A Sequent Calculus is one of the possible calculi one may use to define the concept of provability of First-Order Logic formulas. The first two variants of sequent calculi were invented by Gentzen in 1934 [6], and they were called LK, for classical First-Order Logic, and $L J$, for intuitionistic First-Order Logic.

Here we use a variant of Gentzen's Sequent Calculus LK. The main difference of our variant is the allowance of arbitrary atomic sequents as axiom sequents. This is an important feature, because real mathematical theories usually have a huge number of trivial (from the logical point of view, atomic) axioms, and it would be impractical to carry all these axioms in the antecedent of sequents in our LK-Proofs. ${ }^{1}$. Allowing arbitrary axiom

\footnotetext{
${ }^{1}$ For example, in order to prove a formula $F$ within a theory that assumes the atoms $A$ and $B$ among other formulas $\left(F 1, \ldots, F_{n}\right)$, in traditional Sequent Calculus $\mathbf{L K}$ we would try to find an LK-Proof for the sequent $A, B, F 1, \ldots, F_{n} \vdash F$, and this LK-Proof would repetitively carry the atoms $A$ and $B$ along its branches. With our variant of the calculus $\mathbf{L K}$, we may find an LK-Proof for the sequent $F_{1}, \ldots, F_{n} \vdash F$ which possibly admits the sequents $\vdash A$ and $\vdash B$ as axiom sequents.
} 
sequents in the calculus makes the LK-Proofs shorter and more readable, since they do not display so much trivial logical information.

However, arbitrary axiom sequents also bring two disadvantages. Firstly, derived end-sequents do not directly correspond to valid First-Order Logic formulas anymore, but to First-Order Logic formulas that are valid relatively to the allowed axioms. Secondly, Gentzen's famous cut-elimination theorem holds only in a weaker form. Since his elimination rule for a cut on an axiom requires axiom sequents of the form $A \vdash A$, atomic cuts cannot be eliminated in our variant of the Sequent Calculus LK, which allows arbitrary atomic axiom sequents.

Furthermore we provide definitional and equality rules, which bring the Sequent Calculus closer to real mathematical proofs.

We define our variant of the Sequent Calculus LK incrementally. In the first subsection we give definitions that construct sequents from First-Order Logic formulas. In the second subsection, rules of inference are defined and constructed with sequents, and the admissible schemata of rules of inference in our LK is presented. In the third subsection, LK-Proofs are defined using rules of inference as their building blocks. Finally, a fourth chapter describes notations used to refer to specific parts of an LK-Proof or to denote substitutions of these parts by others.

\subsubsection{Sequents}

Definition 2.3.1 (Sequent). A sequent is a pair of tuples of formulas. The first tuple is the antecedent of the sequent and the second tuple is the consequent of the sequent. We use the notation $A_{1}, \ldots, A_{n} \vdash B_{1}, \ldots, B_{m}$, where $A_{1}, \ldots, A_{n}$ is the antecedent and $B_{1}, \ldots, B_{m}$ is the consequent. The antecedent and the consequent of a sequent may also be empty $(n \geq 0$ and $m \geq 0$ ). If all the formulas in the antecedent and in the consequent are atomic formulas or negated atomic formulas, then the sequent is called atomic.

We use the symbols $\Gamma, \Pi$ and $\Delta$, possibly with subscripts, to denote sequences of formulas in the antecedent and consequent of sequents.

Definition 2.3.2 (Sequent Formation). Given two tuples $A$ and $C$ of formulas, we denote by $\operatorname{seq}(A, C)$ the sequent having $A$ as its antecedent and $C$ as its consequent. Given two sets or multi-sets $S_{A} \doteq\left\{A_{1}, \ldots, A_{n}\right\}$ and $S_{C} \doteq\left\{C_{1}, \ldots, C_{m}\right\}$ of formulas, we denote by $\operatorname{seq}\left(S_{A}, S_{C}\right)$ a permutation variant of the sequent $A_{1}, \ldots, A_{n} \vdash C_{1}, \ldots, C_{m}$.

Definition 2.3.3 (Sequent Composition). Let $s \doteq A_{1}, \ldots, A_{n} \vdash B_{1}, \ldots, B_{m}$ and $s^{\prime} \doteq A_{1}^{\prime}, \ldots, A_{n^{\prime}}^{\prime} \vdash B_{1}^{\prime}, \ldots, B_{m^{\prime}}^{\prime}$. The composition of $s$ and $s^{\prime}$, $A_{1}, \ldots, A_{n}, A_{1}^{\prime}, \ldots, A_{n^{\prime}}^{\prime} \vdash B_{1}, \ldots, B_{m}, B_{1}^{\prime}, \ldots, B_{m^{\prime}}^{\prime}$ is denoted $s \circ s^{\prime}$. 
Definition 2.3.4 (Subsequent). Let $s_{1}$ and $s_{2}$ be sequents. $s_{1}$ is a $s u b$ sequent of $s_{2}$, if and only there is $s^{\prime}$ such that $s_{1} \circ s^{\prime}$ is a permutation variant of $s_{2}$.

Definition 2.3.5 (Positions and Formula Occurrences in a Sequent). Let $s$ be a sequent with $n$ formulas in the antecedent and $m$ formulas in the consequent. A position in a sequent is a string of the form $c . k$ where $c \in$ $\{A, C\}$ and $k$ is a number such that $k \in\{0,1, \ldots, n\}$, if $c=A$, or $k \in$ $\{0,1, \ldots, m\}$, if $c=B$. $c$ indicates whether the position refers to a formula in the antecedent or in the consequent of the sequent, and $k$ indicates the index of the referred formula in the antecedent or in the consequent. By $|s|_{\mu}$ we denote the formula occurring in position $\mu$ of $s$. By convention, $|s|_{A .0} \doteq \mathrm{T}$ and $|s|_{C .0} \doteq \perp$.

Example 2.4 (Positions and Formula Occurrences in a Sequent). Let $s \doteq$ $A, B, C \vdash D, E$. Then:

- $|s|_{A .2}=B$

- $|s|_{A .3}=C$

- $|s|_{C .1}=D$

Definition 2.3.6 (Occurrence Substitution in a Sequent). By $s_{\mu}[B]$ we denote the sequent obtained from $s$ by substituting the formula occurrence in position $\mu$ by $B$. Alternatively, $s_{\mu}[B]$ may also be denoted $s \sigma^{o s}$ with $\sigma^{o s} \doteq\{\mu / B\}$

Example 2.5 (Occurrence Substitution in a Sequent). Let $s \doteq A, B, C \vdash$ $D, E$. Then:

- $s_{A .2}[F]=s\{A .2 / F\}=A, F, C \vdash D, E$

- $s_{A .3}[F]=s\{A .3 / F\}=A, B, F \vdash D, E$

- $s_{C .1}[F]=s\{C .1 / F\}=A, B, C \vdash F, E$

Definition 2.3.7 (Sub-formula Position in a Sequent). Previously defined notations allow us to denote a sub-formula in position $\lambda$ of a formula occurrence in position $\mu$ of a sequent $s$ by the expression $\left.\left.|| s\right|_{\mu}\right|_{\lambda}$. Alternatively we define the following simpler notation to express this same sub-formula: $|s|_{(\mu, \lambda)} \cdot$

Definition 2.3.8 (Sub-formula Substitution in an Occurrence in a Sequent). Previously defined notations allow us to denote the result of substituting a sub-formula in position $\lambda$ of a formula occurrence in position $\mu$ of a sequent $s$ by the formula $A$ by the expression $s_{\mu}\left[\left(|s|_{\mu}\right)_{\lambda}[A]\right]$. Alternatively we define the following two simpler notations to express this same result: 
- $s_{(\mu, \lambda)}[A]$.

- $s \sigma^{f s}$, where $\sigma^{f s} \doteq\{(\mu, \lambda) / A\}$

Definition 2.3.9 (Positive and Negative Occurrences in a Sequent). Let $s=A_{1}, \ldots, A_{n} \vdash B_{1}, \ldots, B_{m}$ be a sequent. We say that $A_{1}, \ldots, A_{n}$ are negative occurrences in $s$ and $B_{1}, \ldots, B_{m}$ are positive occurrences in $s$.

Definition 2.3.10 (Positive and Negative Sub-formulas in a Sequent). Let $s$ be a sequent, $A$ be an occurrence of $s$ and $B$ be a sub-formula of $A$.

- If $A$ occurs positively in $s$ and $B$ is a positive sub-formula of $A$, then $B$ is a positive sub-formula of $s$.

- If $A$ occurs negatively in $s$ and $B$ is a positive sub-formula of $A$, then $B$ is a negative sub-formula of $s$.

- If $A$ occurs positively in $s$ and $B$ is a negative sub-formula of $A$, then $B$ is a negative sub-formula of $s$.

- If $A$ occurs negatively in $s$ and $B$ is a negative sub-formula of $A$, then $B$ is a positive sub-formula of $s$.

Definition 2.3.11 (Strong and Weak Quantifiers in a Sequent). Let $s$ be a sequent, $A$ be an occurrence of $s$ and $Q$ be quantifier in $A$.

- If $A$ occurs positively in $s$ and $Q$ is a strong quantifier in $A$, then $Q$ is a strong quantifier in $s$.

- If $A$ occurs positively in $s$ and $Q$ is a weak quantifier in $A$, then $Q$ is a weak quantifier in $s$.

- If $A$ occurs negatively in $s$ and $Q$ is a strong quantifier in $A$, then $Q$ is a weak quantifier in $s$.

- If $A$ occurs negatively in $s$ and $Q$ is a weak quantifier in $A$, then $Q$ is a strong quantifier in $s$.

\subsubsection{Inference Rules}

Definition 2.3.12 (Rule). A rule of Sequent Calculus can be of any of the three forms below:

$$
\begin{gathered}
\overline{s_{C}} r \\
\frac{s_{P}}{s_{C}} r \\
\frac{s_{P_{1}} \quad s_{P_{2}}}{s_{C}} r
\end{gathered}
$$

In these rule schemes, we say that $s_{C}$ is the conclusion sequent. $s_{P}, s_{P_{1}}, s_{P_{2}}$ are premise sequents. Rules are classified according to the number of premise 
sequents. Rules of the first form are nullary rules. Rules of the second form are unary rules. And rules of the third form are binary rules

The following rules are used in our version of $\mathbf{L K}$ :

1. axiom

$$
\overline{s_{A}} \text { axiom }
$$

where $s_{A}$ is an arbitrary atomic sequent. We call the conclusion sequent of an axiom rule axiom. In particular, axioms of the form $A \vdash A$ for $A$ atomic correspond to logical tautologies ${ }^{2}$.

2. propositional

$$
\begin{gathered}
\frac{\Gamma \vdash \Delta, A \quad \Pi \vdash \Lambda, B}{\Gamma, \Pi \vdash \Delta, \Lambda, A \wedge B} \wedge: r \\
\frac{A, B, \Gamma \vdash \Delta}{A \wedge B, \Gamma \vdash \Delta} \wedge: l \\
\frac{A, \Gamma \vdash \Delta \quad B, \Pi \vdash \Lambda}{A \vee B, \Gamma, \Pi \vdash \Delta \Lambda} \vee: l \\
\frac{\Gamma \vdash \Delta, A}{\Gamma \vdash \Delta, A \vee B} \vee: r 1 \quad \frac{\Gamma \vdash \Delta, A}{\Gamma \vdash \Delta, B \vee A} \vee: r 2 \\
\frac{\Gamma \vdash \Delta, A \quad B, \Pi \vdash \Lambda}{A \rightarrow B, \Gamma, \Pi \vdash \Delta, \Lambda} \rightarrow: l \quad \frac{A, \Gamma \vdash \Delta, B}{\Gamma \vdash \Delta, A \rightarrow B} \rightarrow: r \\
\frac{A, \Gamma \vdash \Delta}{\Gamma \vdash \Delta, \neg A} \neg: r \quad \frac{\Gamma \vdash \Delta, A}{\neg A, \Gamma \vdash \Delta} \neg: l
\end{gathered}
$$

3. first-order

$$
\begin{array}{ll}
\frac{\Gamma \vdash \Delta, A\{x \leftarrow \alpha\}}{\Gamma \vdash \Delta,(\forall x) A} \forall: r & \frac{A\{x \leftarrow t\}, \Gamma \vdash \Delta}{(\forall x) A, \Gamma \vdash \Delta} \forall: l \\
\frac{\Gamma \vdash \Delta, A\{x \leftarrow t\}}{\Gamma \vdash \Delta,(\exists x) A} \exists: r & \frac{A\{x \leftarrow \alpha\}, \Gamma \vdash \Delta}{(\exists x) A, \Gamma \vdash \Delta} \exists: l
\end{array}
$$

For the $\forall: r$ and the $\exists: l$ rules the variable $\alpha$ must not occur in $\Gamma$ nor in $\Delta$ nor in $A$. This is the eigenvariable condition

For the $\forall: l$ and the $\exists: r$ rules the term $t$ must not contain a variable that is bound in $A$.

\footnotetext{
${ }^{2}$ in Gentzen's original sequent calculi, only axioms of the form $F \vdash F$, where $F$ is any arbitrary formula, were allowed.
} 
4. structural

$$
\frac{\Gamma \vdash \Delta}{\Gamma \vdash \Delta, A_{1}, \ldots, A_{n}} w: r \quad \frac{\Gamma \vdash \Delta}{A_{1}, \ldots, A_{n}, \Gamma \vdash \Delta} w: l
$$

where $n>0$

$$
\begin{gathered}
\frac{\Gamma \vdash \Delta, A, \Pi}{\Gamma \vdash \Delta, A, \ldots, A, \Pi} c: r \\
\frac{\Gamma, A, \Pi \vdash \Delta}{\Gamma, A, \ldots, A, \Pi \vdash \Delta} c: l \\
\frac{A_{1}, \ldots, A_{n} \vdash \Delta}{A_{\sigma(1)}, \ldots, A_{\sigma(n)} \vdash \Delta} \pi(\sigma): l \quad \frac{\Gamma \vdash A_{1}, \ldots, A_{n}}{\Gamma \vdash A_{\sigma(1)}, \ldots, A_{\sigma(n)}} \pi(\sigma): r
\end{gathered}
$$

where $\sigma$ is a permutation given as list of cycles

$$
\frac{\Gamma \vdash \Delta, A \quad A, \Pi \vdash \Lambda}{\Gamma, \Pi \vdash \Delta, \Lambda} c u t
$$

\subsubsection{LK-Proofs}

Definition 2.3.13 (LK-Proof). an LK-Proof is a tree in which:

1. Each node is a rule.

2. Leaves are axiom rules.

3. For every node $r$, each of its premise sequents is the conclusion sequent of a direct ancestor ${ }^{3}$ node. of $r$.

Definition 2.3.14 (End-sequent of a proof). The conclusion sequent of the root of a proof $\varphi$ is called end-sequent and is denoted endseq $(\varphi)$.

Definition 2.3.15 (Valid Sequent). A sequent $s$ is said to be a valid sequent, if and only if there is an LK-Proof $\varphi$ such that $\operatorname{endse} q(\varphi)=s$.

Definition 2.3.16 (Alternative view of an LK-Proof). Alternatively and more informally, an LK-Proof can also be viewed as a tree where the nodes are sequents, the leaves are axioms, and the edges are rules connecting the conclusions with the premises.

\footnotetext{
${ }^{3}$ The tree is drawn in the standard way, with the root below and the leaves above. When we say that a node $m$ is the ancestor of a node $n$, we mean that $n$ is in the path between $m$ an the root.
} 
Definition 2.3.17 (Regular Proofs). An LK-Proof is called regular if and only if for each pair of strong quantifier rules $\rho_{1}$ and $\rho_{2}$, the eigen-variable of $\rho_{1}$ is different from the eigen-variable of $\rho_{2}$.

Definition 2.3.18 (Logical Equivalence of Formulas). Two Formulas $A$ and $B$ are said to be equivalent, $A \sim B$, if and only if there is an LK-Proof $\varphi$ such that the end-sequent of $\varphi$ is $\vdash(A \rightarrow B) \wedge(B \rightarrow A)$.

Definition 2.3.19 (Logical Equivalence of Sequents). Let $s \doteq A_{1}, \ldots, A_{n} \vdash$ $B_{1}, \ldots, B_{m}$ and $s^{\prime} \doteq A_{1}^{\prime}, \ldots, A_{j}^{\prime} \vdash B_{1}^{\prime}, \ldots, B_{k}^{\prime}$ be sequents. Then $s$ and $s^{\prime}$ are said to be equivalent, if and only if there is an LK-Proof $\varphi$ such that endseq $(\varphi)=\vdash\left(F_{1} \rightarrow F_{2}\right) \wedge\left(F_{2} \rightarrow F_{1}\right)$, where $F_{1} \doteq\left(\ldots\left(A_{1} \wedge \ldots\right) \wedge A_{n}\right) \rightarrow$ $\left(\ldots\left(B_{1} \vee \ldots\right) \vee B_{m}\right)$ and $F_{2} \doteq\left(\ldots\left(A_{1}^{\prime} \wedge \ldots\right) \wedge A_{j}^{\prime}\right) \rightarrow\left(\ldots\left(B_{1}^{\prime} \vee \ldots\right) \vee B_{k}^{\prime}\right)$.

Definition 2.3.20 (Logical Equivalence between Formulas and Sequences of Formulas). Let $A_{1}, \ldots, A_{n}$ be a sequence of formulas (occurrences or subformulas) in a sequent $s$, and let $A$ be a formula. Then:

- $A_{1}^{+}, \ldots, A_{n}^{+} \sim A$, if and only if $A_{1}, \ldots, A_{n}$ occur positively in $s$ and $A \sim\left(A_{1} \vee\left(\ldots\left(A_{n-1} \vee A_{n}\right)\right)\right)$

- $A_{1}^{-}, \ldots, A_{n}^{-} \sim A$, if and only if $A_{1}, \ldots, A_{n}$ occur negatively in $s$ and $A \sim\left(A_{1} \wedge\left(\ldots\left(A_{n-1} \wedge A_{n}\right)\right)\right)$

\subsubsection{Positions and Substitutions in Proofs}

Definition 2.3.21 (Positions of an LK-Proof). Let $\varphi$ be an LK-Proof. Then its set of positions $\operatorname{LKpos}(\varphi]$ is the smallest set of strings belonging to $\{1,2\}^{*}$ and such that $\operatorname{LKpos}(\varphi)$ satisfies:

- $\varepsilon \in \operatorname{LKpos}(\varphi)$

- If the root of $\varphi$ is a unary rule, then $\left\{1 . \nu \mid \nu \in \operatorname{LKpos}\left(\varphi^{\prime}\right)\right\} \subset \operatorname{LKpos}(\varphi)$ where $\varphi^{\prime}$ is the sub-tree of $\varphi$ having the premise-sequent of the root of $\varphi$ as its end-sequent.

- If the root of $\varphi$ is a binary rule, then $\left\{1 . \nu \mid \nu \in L K \operatorname{pos}\left(\varphi_{1}\right)\right\} \subset L K \operatorname{Los}(\varphi)$ and $\left\{2 . \nu \mid \nu \in \operatorname{LKpos}\left(\varphi_{2}\right)\right\} \subset \operatorname{LKpos}(\varphi)$; where $\varphi_{1}$ is the sub-tree of $\varphi$ having the first premise-sequent of the root of $\varphi$ as its end-sequent, and $\varphi_{2}$ is the sub-tree of $\varphi$ having the second premise-sequent of the root of $\varphi$ as its end-sequent.

Definition 2.3.22 (Depth of a Position of a Proof). Let $\nu$ be a position of a proof $\varphi$. Then, the depth of $\varphi, \operatorname{depth}(\varphi)$ is defined inductively:

1. $\operatorname{depth}(\epsilon) \doteq 0$.

2. $\operatorname{depth}\left(1 . \nu^{\prime}\right) \doteq 1+\operatorname{depth}\left(\nu^{\prime}\right)$. 
3. $\operatorname{depth}\left(2 . \nu^{\prime}\right) \doteq 1+\operatorname{depth}\left(\nu^{\prime}\right)$.

Definition 2.3.23 (Sub-proofs occurring in given Positions). Let $\nu$ be a position of the LK-Proof $\varphi$. Then the sub-proof occurring in position $\nu$ is denoted $|\varphi|_{\nu}$. Formally, we may define the computation of $|\varphi|_{\nu}$ inductively:

1. $|\varphi|_{\varepsilon} \doteq \varphi$

2. If $\nu=1 . \nu^{\prime}$ with $\nu^{\prime} \in \operatorname{pos}\left(\varphi_{1}\right)$, then $|\varphi|_{\nu} \doteq\left|\varphi_{1}\right|_{\nu^{\prime}}$, where $\varphi_{1}$ is the sub-tree of $\varphi$ having the first premise-sequent of the root of $\varphi$ as its end-sequent.

3. If $\nu=2 . \nu^{\prime}$ with $\nu^{\prime} \in \operatorname{pos}\left(\varphi_{2}\right)$, then $|\varphi|_{\nu} \doteq\left|\varphi_{2}\right|_{\nu^{\prime}}$, where $\varphi_{2}$ is the sub-tree of $\varphi$ having the first premise-sequent of the root of $\varphi$ as its end-sequent.

Definition 2.3.24 (Sub-proof Substitution). Let $\varphi$ be an LK-Proof. Then $\varphi_{\nu}\left[\varphi^{\prime}\right]$ denotes $\varphi^{*}$ obtained from $\varphi$ by substituting its sub-proof in position $\nu$ by $\varphi^{\prime} 4$. Alternatively we also define the following notation to express this same $\varphi^{*}: \varphi \sigma^{p p}$, where $\sigma^{p p} \doteq\left\{\nu / \varphi^{\prime}\right\}$.

Definition 2.3.25 (Sequent Position in a Proof). Previously defined notations allow us to denote any sequent in a proof by first obtaining a sub-proof and then extracting the end-sequent of this sub-proof. Thus, a sequent in a Proof $\varphi$, which is the end-sequent of a sub-proof $\varphi^{\prime}$ in position $\nu$ can be denoted by endse $\left(|\varphi|_{\nu}\right)$. Alternatively we define the following simpler notation to express this same sequent: $|\varphi|_{[\nu]}$.

Definition 2.3.26 (Sequent Substitution in a Proof). Let $\varphi$ be an LKProof such that $s \doteq|\varphi|_{[\nu]}$. Then we denote by $\varphi_{[\nu]}\left[s^{\prime}\right]$ the proof $\varphi^{\prime}$ resulting from the substitution of the sequent $s$ in position $\nu$ by the sequent $s^{\prime}$ in the proof $\varphi$. Alternatively we also define the following notation to express this same proof $\varphi^{\prime}: \varphi \sigma^{s p}$, where $\sigma^{s p} \doteq\left\{[\nu] / s^{\prime}\right\}$

Definition 2.3.27 (Occurrence Position in a Proof). Previously defined notations allow us to denote an occurrence in position $\mu$ of an end-sequent $s$ in a sub-proof $\varphi^{\prime}$ in position $\nu$ of a proof $\varphi$ by the expression $\left.\left.|| \varphi\right|_{[\nu]}\right|_{\mu}$. Alternatively we define the following simpler notation to express this same occurrence: $|\varphi|_{[\nu, \mu]}$.

Definition 2.3.28 (Occurrence Substitution in a Proof). Let $\varphi$ be an LKProof such that $F \doteq|\varphi|_{[\nu, \mu]}$. Then we denote by $\varphi_{[\nu, \mu]}\left[F^{\prime}\right]$ the proof $\varphi^{\prime}$ resulting from the substitution of the occurrence $F$ in position $\mu$ of the endsequent of the sub-proof in position $\nu$ by the formula $F^{\prime}$. Alternatively we also define the following notation to express this same proof $\varphi^{\prime}: \varphi \sigma^{o p}$, where $\sigma^{o p} \doteq\left\{[\nu, \mu] / F^{\prime}\right\}$

\footnotetext{
${ }^{4} \varphi_{\nu}\left[\varphi^{\prime}\right]$ will be a valid LK-Proof, only if endseq $\left(\varphi_{\nu}\right)=\operatorname{endseq}\left(\varphi^{\prime}\right)$. Nevertheless, substitutions resulting in invalid proofs can still be useful as intermediary steps in prooftransformation algorithms.
} 
Definition 2.3.29 (Sub-formula Position in a Proof). Previously defined notations allow us to denote a sub-formula in position $\lambda$ of an occurrence in position $\mu$ of an end-sequent $s$ in a sub-proof $\varphi^{\prime}$ in position $\nu$ of a proof $\varphi$ by the expression ||$\left.|\varphi|_{[} \nu\right]\left.\left.\right|_{\mu}\right|_{\lambda}$. Alternatively we define the following simpler notation to express this same sub-formula: $|\varphi|_{[\nu, \mu, \lambda]}$.

Definition 2.3.30 (Sub-formula Substitution in a Proof). Let $\varphi$ be an LKProof such that $A \doteq|\varphi|_{[\nu, \mu, \lambda]}$. Then $\varphi_{[\nu, \mu, \lambda]}\left[A^{\prime}\right]$ denotes the proof $\varphi^{\prime}$ resulting from the substitution of the sub-formula $A$ in position $\lambda$ of the occurrence in position $\mu$ of the end-sequent of the sub-proof in position $\nu$ by the formula $A^{\prime}$. Alternatively we also define the following notation to express this same proof $\varphi^{\prime}: \varphi \sigma^{f p}$, where $\sigma^{f p} \doteq\left\{[\nu, \mu, \lambda] / A^{\prime}\right\}$

\subsubsection{Formula Occurrences in Proofs}

Some occurrences of formulas in proofs are of particular importance, and therefore in this section we define them.

Definition 2.3.31 (Active Occurrences, Main and Auxiliary Occurrences). In the rules of Sequent Calculus some occurrences are written down explicitly with roman letters $(A, B, C, \ldots)$, while others occur implicitly in the context, which is written down with capital greek letters $(\Gamma, \Delta, \Pi, \ldots)$. For a rule $r$ in a proof $\varphi$, the formula occurrence in the conclusion sequent of $r$ which corresponds to the formula written down explicitly in the definition of $r$ is called main occurrence of $r$. The formulas in the premise sequents of $r$ which correspond to formulas written down explicitly in the definition of $r$ are called auxiliary occurrences of $r$. If a formula is a main occurrence or an auxiliary occurrence of $r$, then it is called active occurrence of $r$.

Definition 2.3.32 (Axiom-Occurrence, End-Occurrence, Cut-Occurrence, Terminal Occurrence). A formula occurrence in the conclusion sequent of an axiom rule is called axiom-occurrence. A formula occurrence in the endsequent of a proof is called end-occurrence. An auxiliary occurrence of a cut-rule is called cut-occurrence. If an occurrence is an end-occurrence or a cut-occurrence, then it is called a terminal occurrence.

Definition 2.3.33 (Ancestor-Relation of Occurrences). Let $A$ be an occurrence in a premise-sequent of a rule $r$ in a proof $\varphi$ and let $C$ be an occurrence in the conclusion-sequent of $r$. $A$ is an immediate ancestor of $C$, if $A$ is an auxiliary occurrence of $r$ and $C$ is the main occurrence of $r$ or if $A$ occurs in the context of a premise-sequent of $r$ and $C$ is the corresponding occurrence in the corresponding context in the conclusion-sequent of $r$.

The Ancestor-Relation over occurrences is the transitive closure of the immediate-ancestor-relation. That is, $A_{1}$ is an ancestor of $A_{2}$ if and only if $A_{1}$ is an immediate ancestor of $A_{2}$ or there exists an immediate ancestor $Z$ of $A_{2}$ such that $A_{1}$ is an ancestor of $Z$. 
Table 2.1: Notations for Positions

\begin{tabular}{|c|c|}
\hline Notation & Explanation \\
\hline$|F|_{\lambda}$ & Sub-formula in position $\lambda$ of the Formula $F$ \\
\hline$\overline{|s|_{\mu}}$ & Occurrence in position $\mu$ of the sequent $s$ \\
\hline$|s|_{(\mu, \lambda)}$ & $\begin{array}{l}\text { Sub-formula in position } \lambda \text { of the occurrence in posi- } \\
\text { tion } \mu \text { of the sequent } s\end{array}$ \\
\hline$|\varphi|_{\nu}$ & Sub-proof in position $\nu$ of the proof $\varphi$ \\
\hline$|\varphi|_{[\nu]}$ & $\begin{array}{l}\text { End-sequent of the sub-proof in position } \nu \text { of the } \\
\text { proof } \varphi\end{array}$ \\
\hline$|\varphi|_{[\nu, \mu]}$ & $\begin{array}{l}\text { Occurrence in position } \mu \text { of the end-sequent of the } \\
\text { sub-proof in position } \nu \text { of the proof } \varphi\end{array}$ \\
\hline$|\varphi|_{[\nu, \mu, \lambda]}$ & $\begin{array}{l}\text { Sub-formula in position } \lambda \text { of the occurrence in posi- } \\
\text { tion } \mu \text { of the end-sequent of the sub-proof in position } \\
\nu \text { of the proof } \varphi\end{array}$ \\
\hline
\end{tabular}

Definition 2.3.34 (Used Occurrences). An occurrence $F$ in a proof $\varphi$ is called used if there is an axiom-occurrence which is an ancestor of $F$.

\subsection{Summary of Notations for Positions and Sub- stitutions}

In the previous sections, many notations were defined to access and substitute sub-formulas, occurrences, sequents and sub-proofs in specified positions in occurrences, sequents and proofs. Tables 2.4 and 2.4 provide a summary of all the notations introduced. In this section we also give a general definition of composition of substitutions.

Definition 2.4.1 (Composition of Substitutions). Let $\sigma^{\alpha \beta}$ and $\sigma^{\gamma \beta}$ be substitutions, where $\alpha, \beta$ and $\gamma$ range over $\{f, o, s, p\}$ according to the possibilities displayed in table 2.4. Then $\sigma^{\alpha \beta} \circ \sigma^{\gamma \beta}$ denotes the composition of these substitutions, such that:

$$
Z\left(\sigma^{\alpha \beta} \circ \sigma^{\gamma \beta}\right) \doteq\left(Z \sigma^{\alpha \beta}\right) \sigma^{\gamma \beta}
$$


Table 2.2: Notations for Substitutions

\begin{tabular}{|c|c|l|}
\hline Notation 1 & Notation 2 & Explanation \\
\hline \hline$F_{\lambda}\left[A^{\prime}\right]$ & $F \sigma^{f o}=F\left\{\lambda / A^{\prime}\right\}$ & $\begin{array}{l}\text { Substitution of sub-formula in } \\
\text { position } \lambda \text { of the Formula } F \text { by } \\
A^{\prime}\end{array}$ \\
\hline$s_{\mu}\left[F^{\prime}\right]$ & $s \sigma^{o s}=s\left\{\mu / F^{\prime}\right\}$ & $\begin{array}{l}\text { Substitution of occurrence in } \\
\text { position } \mu \text { of the sequent } s \text { by } \\
F^{\prime}\end{array}$ \\
\hline$s_{(\mu, \lambda)}\left[A^{\prime}\right]$ & $s \sigma^{f s}=s\left\{(\mu, \lambda) / A^{\prime}\right\}$ & $\begin{array}{l}\text { Substitution of sub-formula in } \\
\text { position } \lambda \text { of the occurrence in } \\
\text { position } \mu \text { of the sequent } s \text { by } \\
A^{\prime}\end{array}$ \\
\hline$\varphi_{\nu}\left[\varphi^{\prime}\right]$ & $\varphi \sigma^{p p}=\varphi\left\{\nu / \varphi^{\prime}\right\}$ & $\begin{array}{l}\text { Substitution of sub-proof in } \\
\text { position } \nu \text { of the proof } \varphi \text { by } \\
\varphi^{\prime}\end{array}$ \\
\hline$\varphi_{[\nu]}\left[s^{\prime}\right]$ & $\varphi \sigma^{s p}=\varphi\left\{[\nu] / s^{\prime}\right\}$ & $\begin{array}{l}\text { Substitution of the end- } \\
\text { sequent of the sub-proof in } \\
\text { position } \nu \text { of the proof } \varphi \text { by } s^{\prime}\end{array}$ \\
\hline$\varphi_{[\nu, \mu]}\left[F^{\prime}\right]$ & $\varphi \sigma^{o p}=\varphi\left\{[\nu, \mu] / F^{\prime}\right\}$ & $\begin{array}{l}\text { Substitution of the occurrence } \\
\text { in position } \mu \text { of the end- } \\
\text { sequent of the sub-proof in po- } \\
\text { sition } \nu \text { of the proof } \varphi \text { by } F^{\prime}\end{array}$ \\
\hline$\varphi_{[\nu, \mu, \lambda]}\left[A^{\prime}\right]$ & $\varphi \sigma^{f p}=\varphi\left\{[\nu, \mu, \lambda] / A^{\prime}\right\}$ & $\begin{array}{l}\text { Substitution of the sub- } \\
\text { formula in position } \lambda \text { of the } \\
\text { occurrence in position } \mu \text { of the } \\
\text { end-sequent of the sub-proof } \\
\text { in position } \nu \text { of the proof } \varphi \\
\text { by } A^{\prime}\end{array}$ \\
\hline & &
\end{tabular}




\section{Chapter 3}

\section{Proof Analysis and Proof Transformations}

LK-Proofs satisfying certain syntactical restrictions can be useful for different purposes. One might be interested, for example, in proofs where every sequent has formulas in negation normal form only, or in skolem normal form. Or one might want proofs in cut-normal form, in which the cut-rule is not used.

Given an LK-Proof for a sequent $s$, it is sometimes possible to transform this proof into another proof for $s$ that satisfies the desired syntactical restrictions. In this chapter we define some restrictions and normal forms that are required by some of the algorithms described in subsequent chapters, and we present some theorems regarding transformations that provide LK-Proofs satisfying these restrictions.

\subsection{Prenex Form}

Intuitively, a formula in prenex form is a formula in which all quantifiers are in its beginning. One of the main purposes of the prenex form in our case, is that it is a pre-requisite for certain algorithms, as for example Gentzen's Mid-Sequent Reduction.

Any formula in First-Order Logic can be transformed to an equivalent formula in prenex form. However, this transformation reduces humanunderstandability, because the original positions of the quantifiers, possibly nested within propositional connectives, usually convey a reading of the formula that is closer to the way humans think.

Definition 3.1.1 (Prenex Form). A formula $A$ is in prenex form if and only if it is of the form $\left(Q_{1} x_{1}\right)\left(Q_{2} x_{2}\right) \ldots\left(Q_{n} x_{n}\right) B$, for some $n \geq 0$, for $Q_{1}, \ldots, Q_{n} \in\{\forall, \exists\}$, and for $B$ quantifier-free. A sequent $s$ is in prenex form if and only if all its formulas are in prenex form. An LK-Proof is in prenex form if and only if all its sequents are in prenex form. 
Theorem 3.1 (Transformation into Prenex Form). For any formula $A$, there is a formula $A^{\prime}$ in prenex form and logically equivalent to $A$.

\subsection{Skolemization}

Skolemization is here defined as the process of removing strong quantifiers from First-Order Logic formulas or from sequents. Our definition of Skolemization generalizes more common definitions given in First-Order Logic, in order to make it suitable for Sequent Calculus and for structural skolemization.

Usually, skolemization is defined as the removal of existential quantifiers from First-Order Logic formulas, and dual-skolemization or herbrandization is defined as the removal of universal quantifiers. With these definitions, one can show that, for arbitrary $F_{1}$ and $F_{2}$ in prenex form, skolemization $\left(F_{1}\right) \vdash$ if and only $F_{1} \vdash$ and $\vdash$ dualskolemization $\left(F_{2}\right)$ if and only if $\vdash F_{2}$. Our generalized definition of skolemization corresponds, in the prenex case, to the removal of universal quantifiers (dual-skolemization) in the consequent and to the removal of existential quantifiers (skolemization) in the antecedent. Moreover, in the non-prenex case our definition allows structural skolemization: it does not require the non-prenex formulas or sequents to be firstly transformed to prenex normal form, as it is usually done with the more common definitions; instead it allows the undesired strong quantifiers to be removed in place.

Theorem 3.2 states that Skolemization preserves validity. Therefore, to prove that a sequent $s$ is valid, it suffices to search for an LK-Proof for $S k(s)$. This is good, because Skolemization, for our purposes, has two advantages:

1. An LK-Proof without quantified cut-formulas of a skolemized sequent has neither $(\forall: r)$ nor $(\exists: l)$ rules. Therefore there is no need to worry about eigen-variable conditions.

2. What appears as an eigen-variable in a non-skolemized proof, appears as a term with skolem constants and skolem functions in the corresponding skolemized proof. These terms are usually more humanunderstandable than just eigen-variable, because they show explicit dependencies in their expressions with skolem constants and skolem functions.

Moreover, Structural Skolemization has two advantages over Prenex Skolemization:

1. By leaving the weak quantifiers in their places, the human-readability and the human-understandability of the formula or of the sequent is better preserved. 
2. As shown in [1], Prenex Skolemization may result in a non-elementary increase in the Herbrand Complexity of an LK-Proof.

Below we give formal definitions and about Structural Skolemization of Formulas and Sequents. Algorithms for Structural Skolemization of LKProofs may be found in [10].

Definition 3.2.1 (Skolemization of a First-Order-Logic Formula). Let $F \in$ $F_{F O L}$. Then the Skolemization of $F, S k(F)$ is defined inductively as:

1. If $F$ does not have strong quantifiers, then $S k(F) \doteq F$.

2. If $F$ has strong quantifiers and $(Q y)$ is its first strong quantifier, then:

(a) If $(Q y)$ is not in the scope of weak quantifiers, then $S k(F) \doteq S k\left(F_{-(Q y)}\left\{y \leftarrow c_{y}\right\}\right)$

(b) If $(Q y)$ is in the scope of the weak quantifiers $\left(Q x_{1}\right)\left(Q x_{2}\right) \ldots\left(Q x_{n}\right)$ appearing in this order, then $S k(F) \doteq S k\left(F_{-(Q y)}\left\{y \leftarrow f_{y}\left(x_{1}, x_{2}, \ldots, x_{n}\right)\right\}\right)$

where:

- $c_{y}$ is a constant symbol not occurring in $F$ and is called a skolemconstant.

- $f_{y}$ is a function symbol not occurring in $F$ and is called a skolemfunction.

If the skolem-constant and skolem-function symbols are chosen deterministically in the definition of $S k$, then $S k$ is indeed a function.

Definition 3.2.2 (Set of Skolemizations of a Formula). For different choices of skolem-constant and skolem-function symbols, $S k(F)$ produces different skolemized formulas (which are however equal modulo renaming of the skolem-constant symbols and of the skolem-function symbols). Skolem $(F)$ denotes the set of all the skolemized formulas of $F$ :

$$
\operatorname{Skolem}(F) \doteq\left\{S k_{i}(F) \mid S k_{i} \text { is a skolemization }\right\}
$$

Definition 3.2.3 (Sequent Skolemization). Let $s \doteq A_{1}, \ldots, A_{n} \vdash B_{1}, \ldots, B_{m}$ be a sequent such that $S k\left(A_{1} \wedge \ldots \wedge A_{n} \rightarrow B_{1} \vee \ldots \vee B_{m}\right)=A_{1}^{\prime} \wedge \ldots \wedge A_{n}^{\prime} \rightarrow$ $B_{1}^{\prime} \vee \ldots \vee B_{m}^{\prime}$. Then $S k(s) \doteq A_{1}^{\prime}, \ldots, A_{n}^{\prime} \vdash B_{1}^{\prime}, \ldots, B_{m}^{\prime}$.

Theorem 3.2 (Validity Preservation of Skolemization). Let $s$ be a sequent. $s$ is a valid sequent if and only if $S k(s)$ is a valid sequent. 


\subsection{Herbrand Sequent}

The concept of Herbrand Sequent is the core of this thesis. It allows a certain kind of reduction from First-Order Logic to propositional logic, because a Herbrand sequent for a sequent $s$ contains sufficient instantiations for the quantified variables in $s$. Hence, by extracting a Herbrand Sequent from an LK-Proof, a summarized representation of its creative first-order content is obtained.

Herbrand Sequents, as described here, are a generalization of Herbrand Disjunctions [9] for the Sequent Calculus LK. Apart from the practical use described in this thesis, the concept of Herbrand Disjunction (or Herbrand Sequent) is also very important for the foundations of Logic and Mathematics, by way of Herbrand's Theorem. A concise historical and mathematical discussion of Herbrand's Theorem, as well as its relation to Gödel's Completeness Theorem, may be found in [5].

Herbrand's Theorem, by stating that every valid sequent has a Herbrand sequent, implies that it is always possible to extract Herbrand sequents from proofs, and therefore our general idea of using Herbrand sequents to summarize information about LK-Proofs is applicable to any LK-Proof, even though some of our particular algorithms may impose certain restrictions on the LK-Proofs that they can analyze.

A weaker form of Herbrand's Theorem, restricted to prenex sequents, is equivalent to the famous Mid-Sequent Theorem or Sharpened Hauptsatz, by Gentzen [6]. The proof of this theorem relies on a proof-transformation called Mid-Sequent Reduction, which we use as a first algorithm for the extraction of Herbrand Sequents from prenex LK-Proofs.

Definition 3.3.1 (Herbrand Sequents of a Sequent without Strong Quantifiers). Let $s \doteq A_{1}, \ldots, A_{n} \vdash B_{1}, \ldots, B_{m}$ be a valid sequent containing weak quantifiers only and let $A_{1}^{0}, \ldots, A_{n}^{0}, B_{1}^{0}, \ldots, B_{m}^{0}$ be its formulas without quantifiers. Furthermore, let $A_{j}^{1}, \ldots, A_{j}^{i_{j}}$ be quantifier-free substitution instances of $A_{j}^{0}\left(A_{j}^{h}\right.$, for $0<h \leq i_{j}$, is obtained from $A_{j}^{0}$ by substituting its free variables by terms) and $B_{k}^{1}, \ldots, B_{k}^{p_{k}}$ be quantifier-free substitution instances of $B_{k}^{0}$. A valid sequent of the form

$$
s^{\prime}=A_{1}^{1}, \ldots, A_{1}^{i_{1}}, \ldots A_{n}^{1}, \ldots, A_{n}^{i_{n}} \vdash B_{1}^{1}, \ldots, B_{1}^{p_{1}}, \ldots, B_{m}^{1}, \ldots, B_{m}^{p_{m}}
$$

is called a Herbrand Sequent of $s$.

Clearly, there are infinitely many such sequents and $H(s)$ denotes the set of all Herbrand sequents of $s$.

Example 3.1 (Herbrand Sequent of a Sequent). Let $s \doteq P(0),(\forall x)(P(x) \rightarrow$ $P(s(x))) \vdash P(s(s(0)))$. Then some Herbrand Sequents of $s$ are:

- $P(0), P(0) \rightarrow P(s(0)), P(s(0)) \rightarrow P(s(s(0))) \vdash P(s(s(0)))$

- $P(0), P(0), P(0) \rightarrow P(s(0)), P(s(0)) \rightarrow P(s(s(0))) \vdash P(s(s(0)))$ 
- $P(0), P(0) \rightarrow P(s(0)), P(s(0)) \rightarrow P(s(s(0))), P(s(s(0))) \rightarrow P(s(s(s(0)))) \vdash P(s(s(0)))$

- ....

Definition 3.3.2 (Herbrand Sequents of a Sequent). Let $s$ be an arbitrary sequent. We define the set of its Herbrand sequents:

$$
H(s) \doteq \bigcup_{s^{\prime} \in \operatorname{Skolem}(s)} H\left(s^{\prime}\right)
$$

If $s^{*} \in H(s)$, then $s^{*}$ is called a Herbrand Sequent of $s$.

Theorem 3.3 (Herbrand's Theorem). A sequent $s$ is valid if and only if there exists a Herbrand sequent of $s$.

Proof. Originally in [9], stated for Herbrand Disjunctions. Also in [5] with more modern proof calculi.

Theorem 3.4 (Mid-Sequent Theorem or Sharpened Hauptsatz). Let $\varphi$ be a prenex LK-Proof without non-atomic cuts. Then there is an LK-Proof $\varphi^{\prime}$ of the same end-sequent such that no quantifier rule occurs above propositional and cut rules.

Proof. Original proof, for Gentzen's original Sequent Calculus LK and cutfree LK-Proofs, is available in [6].

\subsection{Cut-Elimination}

The importance of Cut-Elimination, a proof transformation invented by Gentzen [6], for the automatic analysis of mathematical proofs lies on the fact that the removal of cuts from formal LK-Proofs corresponds to the elimination of lemmas from informal mathematical proofs. The resulting cut-free LK-Proof has the property that all the formulas in its sequents are sub-formulas of formulas in the end-sequent, thus making implicit parameters explicit.

The main result, regarding cut-elimination is Gentzen's Hauptsatz or Cut-Elimination Theorem [6], which states that any LK-Proof can be transformed to a cut-free LK-Proof with the same end-sequent. However, since our variant of the Sequent Calculus LK allows arbitrary axiom sequents, Gentzen's Hauptsatz holds only in a weaker form, because his elimination rule for a cut on an axiom requires axiom sequents of the form $A \vdash A$. Our variant of $\mathbf{L K}$ admits transformation to proofs without non-atomic cuts, but in general atomic cuts cannot be eliminated ${ }^{1}$.

\footnotetext{
${ }^{1}$ A simple example of an LK-Proof in our variant of the Sequent Calculus LK which does not admit a transformation to a cut-free proof is:
} 
Although Gentzen's method for Cut-Elimination was the first one, others exist, as for example Cut-Elimination by Resolution (CERes) [?] and CutElimination by Projection [8]. Cut-Elimination is not unique, and therefore different methods or choices within these methods may lead to new interesting proofs.

The importance of cut-elimination for this thesis lies on the fact that all algorithms require LK-Proofs without non-atomic cuts.

Theorem 3.5 (Weak Cut-Elimination Theorem). Let $\varphi$ be an LK-Proof. Then there exists an LK-Proof $\varphi^{\prime}$ without non-atomic cuts such that $\operatorname{endseq}(\varphi)=\operatorname{endseq}\left(\varphi^{\prime}\right)$.

Proof. A proof for the original Cut-Elimination Theorem can be found in $[6]$. 


\section{Chapter 4}

\section{Extraction of Herbrand Sequents}

Four algorithms for the Extraction of Herbrand Sequents from LK-Proofs are described in this chapter. Each algorithm is described in its own subsection, each organized as follows:

- Firstly, necessary notations and definitions specific for the algorithm, are introduced.

- By using all the previously defined mathematical notations and definitions, the algorithm is formally and functionally stated.

- Since the formal functional description of the algorithm is sometimes difficult to understand, a high-level and imperative pseudo-code for the algorithm is provided.

- The soundness of the algorithm is proved. This proof is divided in two lemmas: a proof that the extracted sequent is well-instantiated and a proof that the extracted sequent is valid.

- One or more examples are presented, showing how to apply the algorithm to specific LK-Proofs.

The algorithms may be classified on whether they require the LK-Proofs to be in Prenex form and on whether they need to perform transformations in order to extract the Herbrand sequent. Table 4 summarizes this classification.

Throughout this chapter, LK-Proofs are assumed to be free of nonatomic cuts. Thanks to theorem 3.5, a weaker form of Gentzen's CutElimination Theorem, this in principle does not limit the applicability of these algorithms, because proofs could have their non-atomic cuts eliminated. 
Table 4.1: Classification of the Algorithms

\begin{tabular}{|l|l|l|}
\hline & $\begin{array}{l}\text { Prenex LK-Proofs } \\
\text { Required }\end{array}$ & $\begin{array}{l}\text { Prenex LK-Proofs } \\
\text { not Required }\end{array}$ \\
\hline $\begin{array}{l}\text { Proof- } \\
\text { Transformations } \\
\text { Used }\end{array}$ & $\begin{array}{l}\text { Mid-Sequent Reduc- } \\
\text { tion }\end{array}$ & $\begin{array}{l}\text { Transformation to } \\
\text { LK }_{\text {A }}\end{array}$ \\
\hline $\begin{array}{l}\text { Proof- } \\
\text { Transformations } \\
\text { not Used }\end{array}$ & Collection of Instances & $\begin{array}{l}\text { Collection of Sub- } \\
\text { formula Instances }\end{array}$ \\
\hline
\end{tabular}




\subsection{Extraction from Prenex Proofs}

The extraction of a Herbrand sequent in the case of LK-Proofs in prenex form is easier than in the more general case of LK-Proofs that are not in prenex form, and it can be done by the simpler algorithms described in the next two subsections.

By theorem 3.1, these two algorithms could in principle also be applied to non-prenex LK-Proofs by firstly transforming them to prenex form. However, this has disadvantages in terms of human-understandability and complexity, and therefore the two algorithms described in section 4.2 should be preferred for the general (not necessarily prenex) case. Nevertheless, the algorithms described in this section form the basis for the understanding of the more complex algorithms of the next section. 


\subsubsection{Extraction via Mid-Sequent Reduction}

The algorithm described in this subsection is based on a mid-sequent reduction explained in [7] and similar to the one described by Gentzen in his proof of the sharpened Hauptsatz (Mid-Sequent Theorem) in [6]. The essential idea of the algorithm is to transform an LK-Proof into a proof in which no propositional-rules or cut-rules appear below quantifier-rules. Such a proof has a so-called mid-sequent, located between the lower part of the proof, which has the quantifier-rules, and the upper part of the proof, which has the propositional rules.

This algorithm is slightly different from the algorithm in [7], because here we allow proofs with atomic cuts, while there they require the proof to be cut-free. Unless stated otherwise, we assume LK-Proofs in this subsection to be free of non-atomic cuts.

Definition 4.1.1 (Degree of a Quantifier-rule). Let $\rho$ be a quantifier rule in an LK-Proof $\varphi$. Then the degree of $\rho$, degree $(\rho)$, is defined as the number of propositional-rules and cut-rules in the path from $\rho$ to the root of $\varphi$.

Definition 4.1.2 (Associated Contraction). Let $\varphi$ be an LK-Proof containing a quantifier-rule $\rho$ with main occurrence $\theta$. Every contraction-rule below $\rho$ that has an auxiliary occurrence $\theta^{\prime}$ such that $\theta$ is ancestor of $\theta^{\prime}$ and the only active formula occurrences this ancestor path passes through are active formula occurrences of contraction-rules, is said to be associated with $\rho$.

Example 4.1 (Associated Contractions). Let $\varphi$ be the LK-Proof below:

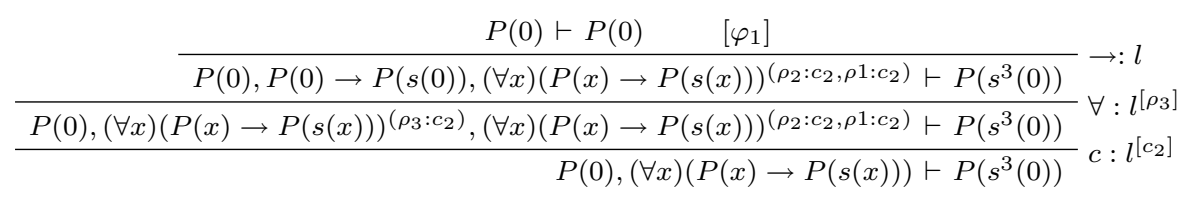

where $\varphi_{1}$ is:

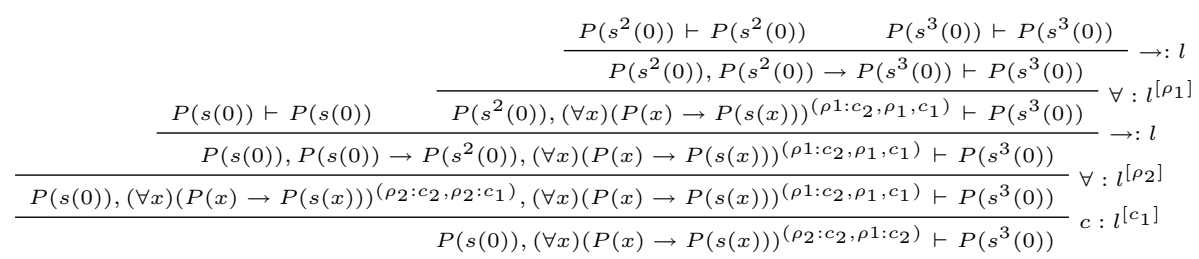

In this proof occurrences have been labeled with $\left(\rho_{j}: c_{i}\right)$ to indicate that they belong to the ancestor path that associates $c_{i}$ to $\rho_{j}$. Therefore the following associations exist in this proof:

- the contractions associated with $\rho_{1}$ are $c_{1}$ and $c_{2}$.

- the contractions associated with $\rho_{2}$ are $c_{1}$ and $c_{2}$. 
- the contraction associated with $\rho_{3}$ is $c_{2}$.

Definition 4.1.3 (Mid-Sequent Reduction). Let $\varphi$ be an LK-Proof. We define the transformation $\rightarrow_{M}$ pushing downwards a non-deterministically chosen quantifier-rule $\rho$ with $\operatorname{degree}(\rho)>0$, according to the following cases:

1. $\rho$ is a $(\forall: r)$-rule: Let $\tau$ be the first propositional or cut-rule below $\rho$ and assume that there is no quantifier rule between $\rho$ and $\tau$ (if this is not the case, choose the quantifier rule that is in between, instead of $\rho$, to push downwards).

(a) $\tau$ is a unary propositional-rule: Then the subproof $\chi$ of $\varphi$ with root $\tau$ has the following form:

$$
\begin{aligned}
& {[\psi]} \\
& \frac{\Gamma \vdash \Delta, F\{x \leftarrow \alpha\}}{\Gamma \vdash \Delta,(\forall x) F} \forall: r^{[\rho]} \\
& \frac{\Gamma^{\prime} \vdash \Delta^{\prime},(\forall x) F}{\Gamma^{\prime \prime} \vdash \Delta^{\prime \prime},(\forall x) F} *: *, * *^{[\delta]}
\end{aligned}
$$

The premise of $\tau$ contains $(\forall x) F$ (as a descendant of the main occurrence of $\rho$ ) because neither contractions nor weakenings can remove it. The propositional rule $\tau$ does not have this $(\forall x) F$ as auxiliary formula (for assuming it has would lead to contradiction, because the main formula of $\tau$ wouldn't be in prenex form). Hence the conclusion of $\tau$ also contains this $(\forall x) F$.

Let $\delta^{\prime}$ be the contractions in $\delta$ that are associated with $\rho$ and let $\Pi$ be the multi-set that contains the formula $(\forall x) F$ exactly $n$ times where $n$ is the number of contractions in $\delta^{\prime}$.

We define $\chi \rightarrow_{M} \chi^{\prime}$ with $\chi^{\prime}$ as shown below:

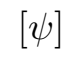

$$
\begin{aligned}
& \begin{array}{l}
\frac{\Gamma \vdash \Delta, F\{x \leftarrow \alpha\}}{\Gamma^{\prime} \vdash \Delta^{\prime}, \Pi, F\{x \leftarrow \alpha\}} c: *, w: *{ }^{\left[\delta \backslash \delta^{\prime}\right]} \\
\frac{\Gamma^{\prime \prime} \vdash \Delta^{\prime \prime}, \Pi, F\{x \leftarrow \alpha\}}{\Gamma^{\prime \prime} \vdash \Delta^{\prime \prime}, \Pi,(\forall x) F} * *^{[\tau]} \\
\left.\frac{\Gamma^{\prime \prime} \vdash \Delta^{\prime \prime},(\forall x) F}{} c: *^{[\rho]}\right] \\
\left.\delta^{\prime}\right]
\end{array}
\end{aligned}
$$

(b) $\tau$ is a binary rule and $\rho$ is in the left branch: Then the subproof $\chi$ of $\varphi$ with root $\tau$ has the following form:

$$
\begin{aligned}
& {[\psi]} \\
& \begin{array}{l}
\frac{\Gamma \vdash \Delta, F\{x \leftarrow \alpha\}}{\Gamma \vdash \Delta,(\forall x) F} \forall: r^{[\rho]} \\
\frac{\Gamma^{\prime} \vdash \Delta^{\prime},(\forall x) F}{\Gamma^{\prime \prime}, \Gamma_{1}^{\prime \prime} \vdash \Delta^{\prime \prime}, \Delta_{1}^{\prime \prime},(\forall x) F} \quad \Gamma_{1}^{\prime} \vdash \Delta_{1}^{\prime} \\
*: *[\tau]
\end{array}
\end{aligned}
$$


The left premise of $\tau$ contains $(\forall x) F$ (as a descendant of the main occurrence of $\rho$ ) because neither contractions nor weakenings can remove it. The propositional rule $\tau$ does not have this $(\forall x) F$ as auxiliary formula (for assuming it has would lead to contradiction, because the main formula of $\tau$ wouldn't be in prenex form). Hence the conclusion of $\tau$ also contains this $(\forall x) F$.

Let $\delta^{\prime}$ be the contractions in $\delta$ that are associated with $\rho$ and let $\Pi$ be the multi-set that contains the formula $(\forall x) F$ exactly $n$ times where $n$ is the number of contractions in $\delta^{\prime}$.

We define $\chi \rightarrow_{M} \chi^{\prime}$ with $\chi^{\prime}$ as shown below:

$$
\begin{aligned}
& {[\psi]}
\end{aligned}
$$

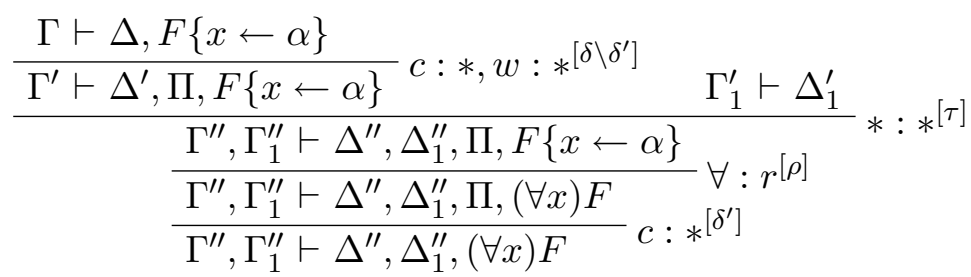

(c) $\tau$ is a binary rule and $\rho$ is in the right branch: Then the subproof $\chi$ of $\varphi$ with root $\tau$ has the following form:

$$
\begin{gathered}
{[\psi]} \\
\frac{\Gamma \vdash \Delta, F\{x \leftarrow \alpha\}}{\Gamma \vdash \Delta,(\forall x) F} \forall: r^{[\rho]} \\
\frac{\Gamma_{1}^{\prime} \vdash \Delta_{1}^{\prime}}{\Gamma^{\prime \prime}, \Gamma_{1}^{\prime \prime} \vdash \Delta^{\prime \prime}, \Delta_{1}^{\prime \prime},(\forall x) F} *: * *^{[\delta]} \\
\Gamma^{\prime} \vdash \Delta^{\prime},(\forall x) F
\end{gathered}
$$

The right premise of $\tau$ contains $(\forall x) F$ (as a descendant of the main occurrence of $\rho$ ) because neither contractions nor weakenings can remove it. The propositional rule $\tau$ does not have this $(\forall x) F$ as auxiliary formula (for assuming it has would lead to contradiction, because the main formula of $\tau$ wouldn't be in prenex form). Hence the conclusion of $\tau$ also contains this $(\forall x) F$.

Let $\delta^{\prime}$ be the contractions in $\delta$ that are associated with $\rho$ and let $\Pi$ be the multi-set that contains the formula $(\forall x) F$ exactly $n$ times where $n$ is the number of contractions in $\delta^{\prime}$.

We define $\chi \rightarrow_{M} \chi^{\prime}$ with $\chi^{\prime}$ as shown below:

$$
\begin{gathered}
{[\psi]} \\
\frac{\Gamma \vdash \Delta, F\{x \leftarrow \alpha\}}{\Gamma_{1}^{\prime} \vdash \Delta_{1}^{\prime} \quad \frac{\Gamma^{\prime} \vdash \Delta^{\prime}, \Pi, F\{x \leftarrow \alpha\}}{\Gamma^{\prime \prime}, \Gamma_{1}^{\prime \prime} \vdash \Delta^{\prime \prime}, \Delta_{1}^{\prime \prime}, \Pi, F\{x \leftarrow \alpha\}}} *: *, w: *^{\left[\delta \backslash \delta^{\prime}\right]} \\
\frac{\Gamma^{\prime \prime}, \Gamma_{1}^{\prime \prime} \vdash \Delta^{\prime \prime}, \Delta_{1}^{\prime \prime}, \Pi,(\forall x) F}{\Gamma^{\prime \prime}, \Gamma_{1}^{\prime \prime} \vdash \Delta^{\prime \prime}, \Delta_{1}^{\prime \prime},(\forall x) F} c: * *^{\left[\delta^{\prime}\right]} \\
r^{[\rho]}
\end{gathered}
$$


The eigen-variable condition of $\rho$ in $\chi^{\prime}$ is fulfilled because $\alpha$ does not occur in $\Gamma, \Delta, \Gamma^{\prime}, \Delta^{\prime}$. In particular, $\alpha$ is not added by a weakening in $\delta$ because the proof is regular.

2. $\rho$ is a $(\exists: r)$-rule: Let $\tau$ be the first propositional or cut-rule below $\rho$ and assume that there is no quantifier rule between $\rho$ and $\tau$ (if this is not the case, choose the quantifier rule that is in between, instead of $\rho$, to push downwards).

(a) $\tau$ is a unary propositional-rule: Then the subproof $\chi$ of $\varphi$ with root $\tau$ has the following form:

$$
\begin{aligned}
& {[\psi]} \\
& \frac{\Gamma \vdash \Delta, F\{x \leftarrow t\}}{\Gamma \vdash \Delta,(\exists x) F} \exists: r^{[\rho]} \\
& \frac{\Gamma^{\prime} \vdash \Delta^{\prime},(\exists x) F}{\Gamma^{\prime \prime} \vdash \Delta^{\prime \prime},(\exists x) F} *: *, *^{[\tau]}
\end{aligned}
$$

The premise of $\tau$ contains ( $\exists x) F$ (as a descendant of the main occurrence of $\rho$ ) because neither contractions nor weakenings can remove it. The propositional rule $\tau$ does not have this $(\exists x) F$ as auxiliary formula (for assuming it has would lead to contradiction, because the main formula of $\tau$ wouldn't be in prenex form). Hence the conclusion of $\tau$ also contains this $(\exists x) F$.

Let $\delta^{\prime}$ be the contractions in $\delta$ that are associated with $\rho$ and let $\Pi$ be the multi-set that contains the formula $(\exists x) F$ exactly $n$ times where $n$ is the number of contractions in $\delta^{\prime}$.

We define $\chi \rightarrow_{M} \chi^{\prime}$ with $\chi^{\prime}$ as shown below:

$$
\begin{aligned}
& {[\psi]} \\
& \frac{\Gamma \vdash \Delta, F\{x \leftarrow t\}}{\Gamma^{\prime} \vdash \Delta^{\prime}, \Pi, F\{x \leftarrow t\}} c: *, w: *^{\left[\delta \backslash \delta^{\prime}\right]} \\
& \frac{\Gamma^{\prime \prime} \vdash \Delta^{\prime \prime}, \Pi, F\{x \leftarrow t\}}{\Gamma^{\prime \prime} \vdash \Delta^{\prime \prime}, \Pi,(\exists x) F} \cdot \exists: *^{[\rho]} \\
& \frac{[\rho]}{\Gamma^{\prime \prime} \vdash \Delta^{\prime \prime},(\exists x) F} c: *^{\left[\delta^{\prime}\right]}
\end{aligned}
$$

(b) $\tau$ is a binary rule and $\rho$ is in the left branch: Then the subproof $\chi$ of $\varphi$ with root $\tau$ has the following form:

$$
\begin{aligned}
& {[\psi]}
\end{aligned}
$$

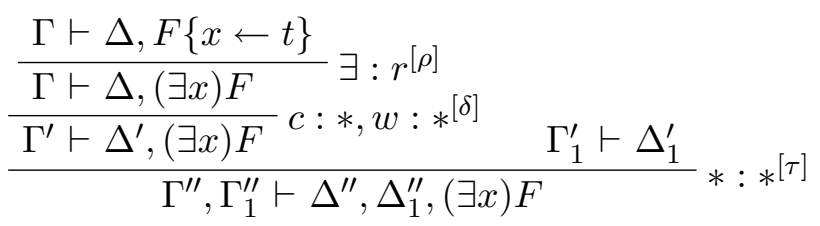


The left premise of $\tau$ contains $(\exists x) F$ (as a descendant of the main occurrence of $\rho$ ) because neither contractions nor weakenings can remove it. The propositional rule $\tau$ does not have this $(\exists x) F$ as auxiliary formula (for assuming it has would lead to contradiction, because the main formula of $\tau$ wouldn't be in prenex form). Hence the conclusion of $\tau$ also contains this $(\exists x) F$.

Let $\delta^{\prime}$ be the contractions in $\delta$ that are associated with $\rho$ and let $\Pi$ be the multi-set that contains the formula $(\exists x) F$ exactly $n$ times where $n$ is the number of contractions in $\delta^{\prime}$.

We define $\chi \rightarrow_{M} \chi^{\prime}$ with $\chi^{\prime}$ as shown below:

$$
\begin{aligned}
& {[\psi]}
\end{aligned}
$$

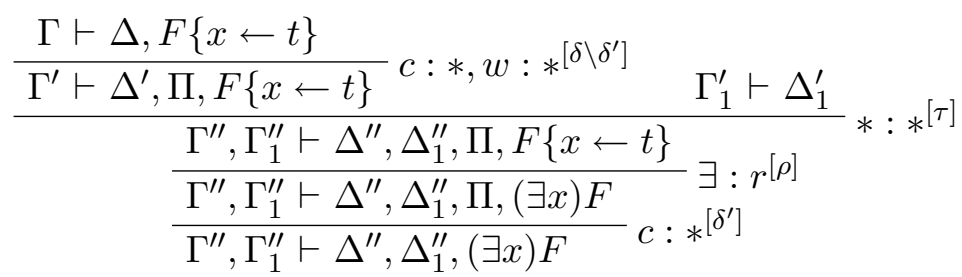

(c) $\tau$ is a binary rule and $\rho$ is in the right branch: Then the subproof $\chi$ of $\varphi$ with root $\tau$ has the following form:

$$
\begin{gathered}
{[\psi]} \\
\frac{\Gamma \vdash \Delta, F\{x \leftarrow t\}}{\Gamma \vdash \Delta,(\exists x) F} \exists: r^{[\rho]} \\
\frac{\Gamma_{1}^{\prime} \vdash \Delta_{1}^{\prime}}{\Gamma^{\prime \prime}, \Gamma_{1}^{\prime \prime} \vdash \Delta^{\prime \prime}, \Delta_{1}^{\prime \prime},(\exists x) F} *: * *^{[\delta]} \\
\Gamma^{\prime} \vdash \Delta^{\prime},(\exists x) F
\end{gathered}
$$

The right premise of $\tau$ contains $(\exists x) F$ (as a descendant of the main occurrence of $\rho$ ) because neither contractions nor weakenings can remove it. The propositional rule $\tau$ does not have this $(\exists x) F$ as auxiliary formula (for assuming it has would lead to contradiction, because the main formula of $\tau$ wouldn't be in prenex form). Hence the conclusion of $\tau$ also contains this $(\exists x) F$.

Let $\delta^{\prime}$ be the contractions in $\delta$ that are associated with $\rho$ and let $\Pi$ be the multi-set that contains the formula $(\exists x) F$ exactly $n$ times where $n$ is the number of contractions in $\delta^{\prime}$.

We define $\chi \rightarrow_{M} \chi^{\prime}$ with $\chi^{\prime}$ as shown below:

$$
\begin{aligned}
& {[\psi]} \\
& \begin{array}{l}
\frac{\Gamma \vdash \Delta, F\{x \leftarrow t\}}{\Gamma_{1}^{\prime} \vdash \Delta_{1}^{\prime} \quad \frac{\Gamma^{\prime} \vdash \Delta^{\prime}, \Pi, F\{x \leftarrow t\}}{\Gamma^{\prime \prime}, \Gamma_{1}^{\prime \prime} \vdash \Delta^{\prime \prime}, \Delta_{1}^{\prime \prime}, \Pi, F\{x \leftarrow t\}}} c: *, w: *^{\left[\delta \backslash \delta^{\prime}\right]} \\
\left.\frac{\Gamma^{\prime \prime}, \Gamma_{1}^{\prime \prime} \vdash \Delta^{\prime \prime}, \Delta_{1}^{\prime \prime}, \Pi,(\exists x) F}{\Gamma^{\prime \prime}, \Gamma_{1}^{\prime \prime} \vdash \Delta^{\prime \prime}, \Delta_{1}^{\prime \prime},(\exists x) F} c: *^{[\rho]}\right] \\
\left.\delta^{[}\right]
\end{array}
\end{aligned}
$$


3. $\rho$ is $a(\forall: l)$-rule: Let $\tau$ be the first propositional or cut-rule below $\rho$ and assume that there is no quantifier rule between $\rho$ and $\tau$ (if this is not the case, choose the quantifier rule that is in between, instead of $\rho$, to push downwards).

(a) $\tau$ is a unary propositional-rule: Then the subproof $\chi$ of $\varphi$ with root $\tau$ has the following form:

$$
\begin{gathered}
{[\psi]} \\
\frac{\Gamma, F\{x \leftarrow t\} \vdash \Delta}{\Gamma,(\forall x) F \vdash \Delta} \forall: l^{[\rho]} \\
\frac{\Gamma^{\prime},(\forall x) F \vdash \Delta^{\prime}}{\Gamma^{\prime \prime},(\forall x) F \vdash \Delta^{\prime \prime}} *: * *^{[\tau]}:{ }^{[\delta]}
\end{gathered}
$$

The premise of $\tau$ contains $(\forall x) F$ (as a descendant of the main occurrence of $\rho$ ) because neither contractions nor weakenings can remove it. The propositional rule $\tau$ does not have this $(\forall x) F$ as auxiliary formula (for assuming it has would lead to contradiction, because the main formula of $\tau$ wouldn't be in prenex form). Hence the conclusion of $\tau$ also contains this $(\forall x) F$.

Let $\delta^{\prime}$ be the contractions in $\delta$ that are associated with $\rho$ and let $\Pi$ be the multi-set that contains the formula $(\forall x) F$ exactly $n$ times where $n$ is the number of contractions in $\delta^{\prime}$.

We define $\chi \rightarrow_{M} \chi^{\prime}$ with $\chi^{\prime}$ as shown below:

$$
\begin{gathered}
{[\psi]} \\
\frac{\Gamma, F\{x \leftarrow t\} \vdash \Delta}{\frac{\Gamma^{\prime}, F\{x \leftarrow t\}, \Pi \vdash \Delta^{\prime}}{\Gamma^{\prime \prime}, F\{x \leftarrow t\}, \Pi \vdash \Delta^{\prime \prime}}} c: *, w: * *^{[\tau]}\left[\delta \backslash \delta^{\prime}\right] \\
\frac{\Gamma^{\prime \prime},(\forall x) F, \Pi \vdash \Delta^{\prime \prime}}{\Gamma^{\prime \prime},(\forall x) F \vdash \Delta^{\prime \prime}} c: *^{[\rho]}\left[\delta^{\prime}\right]
\end{gathered}
$$

(b) $\tau$ is a binary rule and $\rho$ is in the left branch: Then the subproof $\chi$ of $\varphi$ with root $\tau$ has the following form:

$$
\begin{aligned}
& {[\psi]}
\end{aligned}
$$

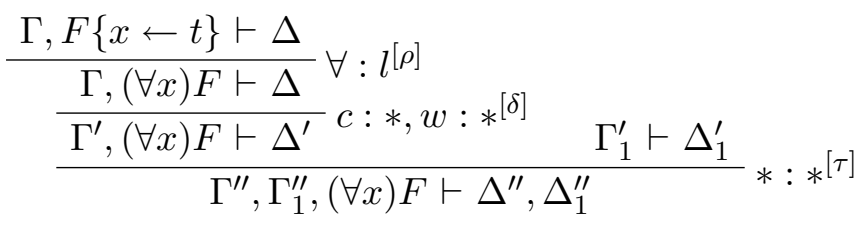

The left premise of $\tau$ contains $(\forall x) F$ (as a descendant of the main occurrence of $\rho$ ) because neither contractions nor weakenings can remove it. The propositional rule $\tau$ does not have this $(\forall x) F$ as auxiliary formula (for assuming it has would lead to contradiction, 
because the main formula of $\tau$ wouldn't be in prenex form). Hence the conclusion of $\tau$ also contains this $(\forall x) F$.

Let $\delta^{\prime}$ be the contractions in $\delta$ that are associated with $\rho$ and let $\Pi$ be the multi-set that contains the formula $(\forall x) F$ exactly $n$ times where $n$ is the number of contractions in $\delta^{\prime}$.

We define $\chi \rightarrow_{M} \chi^{\prime}$ with $\chi^{\prime}$ as shown below:

$$
\frac{[\psi]}{\frac{\Gamma, F\{x \leftarrow t\} \vdash \Delta}{\Gamma^{\prime}, F\{x \leftarrow t\}, \Pi \vdash \Delta^{\prime}} c: *, w: * *^{\left[\delta \backslash \delta^{\prime}\right]} \quad \Gamma_{1}^{\prime} \vdash \Delta_{1}^{\prime}} *_{*}^{[\tau]}
$$

(c) $\tau$ is a binary rule and $\rho$ is in the right branch: Then the subproof $\chi$ of $\varphi$ with root $\tau$ has the following form:

$$
\begin{gathered}
{[\psi]} \\
\frac{\Gamma, F\{x \leftarrow t\} \vdash \Delta}{\Gamma,(\forall x) F \vdash \Delta} \\
\frac{\Gamma_{1}^{\prime} \vdash \Delta_{1}^{\prime}}{\Gamma^{\prime \prime}, \Gamma_{1}^{\prime \prime},(\forall x) F \vdash \Delta^{\prime \prime}, \Delta_{1}^{\prime \prime}} * l^{[\rho]} \\
\Gamma^{\prime},(\forall x) F, w: *[\delta]
\end{gathered}
$$

The right premise of $\tau$ contains $(\forall x) F$ (as a descendant of the main occurrence of $\rho$ ) because neither contractions nor weakenings can remove it. The propositional rule $\tau$ does not have this $(\forall x) F$ as auxiliary formula (for assuming it has would lead to contradiction, because the main formula of $\tau$ wouldn't be in prenex form). Hence the conclusion of $\tau$ also contains this $(\forall x) F$.

Let $\delta^{\prime}$ be the contractions in $\delta$ that are associated with $\rho$ and let $\Pi$ be the multi-set that contains the formula $(\forall x) F$ exactly $n$ times where $n$ is the number of contractions in $\delta^{\prime}$.

We define $\chi \rightarrow_{M} \chi^{\prime}$ with $\chi^{\prime}$ as shown below:

$$
\begin{gathered}
{[\psi]} \\
\frac{\Gamma, F\{x \leftarrow t\} \vdash \Delta}{\Gamma_{1}^{\prime} \vdash \Delta_{1}^{\prime} \quad \frac{\Gamma{ }^{\prime}, F\{x \leftarrow t\}, \Pi \vdash \Delta^{\prime}}{\Gamma^{\prime \prime}, \Gamma_{1}^{\prime \prime}, F\{x \leftarrow t\}, \Pi \vdash \Delta^{\prime \prime}, \Delta_{1}^{\prime \prime}}} * * *, w: *[\delta] \\
\frac{\Gamma^{\prime \prime}, \Gamma_{1}^{\prime \prime},(\forall x) F, \Pi \vdash \Delta^{\prime \prime}, \Delta_{1}^{\prime \prime}}{\Gamma^{\prime \prime}, \Gamma_{1}^{\prime \prime},(\forall x) F \vdash \Delta^{\prime \prime}, \Delta_{1}^{\prime \prime}} c: l^{[\rho]} \\
{\left[\delta^{\prime}\right]}
\end{gathered}
$$

4. $\rho$ is a $(\exists: l)$-rule: Let $\tau$ be the first propositional or cut-rule below $\rho$ and assume that there is no quantifier rule between $\rho$ and $\tau$ (if this is not the case, choose the quantifier rule that is in between, instead of $\rho$, to push downwards). 
(a) $\tau$ is a unary propositional-rule: Then the subproof $\chi$ of $\varphi$ with root $\tau$ has the following form:

$$
\begin{gathered}
{[\psi]} \\
\frac{\Gamma, F\{x \leftarrow \alpha\} \vdash \Delta}{\Gamma,(\exists x) F \vdash \Delta} \exists: l^{[\rho]} \\
\frac{\Gamma^{\prime},(\exists x) F \vdash \Delta^{\prime}}{\Gamma^{\prime \prime},(\exists x) F \vdash \Delta^{\prime \prime}} *: * *^{[\tau]}: *^{[\delta]}
\end{gathered}
$$

The premise of $\tau$ contains $(\exists x) F$ (as a descendant of the main occurrence of $\rho$ ) because neither contractions nor weakenings can remove it. The propositional rule $\tau$ does not have this $(\exists x) F$ as auxiliary formula (for assuming it has would lead to contradiction, because the main formula of $\tau$ wouldn't be in prenex form). Hence the conclusion of $\tau$ also contains this $(\exists x) F$.

Let $\delta^{\prime}$ be the contractions in $\delta$ that are associated with $\rho$ and let $\Pi$ be the multi-set that contains the formula $(\exists x) F$ exactly $n$ times where $n$ is the number of contractions in $\delta^{\prime}$.

We define $\chi \rightarrow_{M} \chi^{\prime}$ with $\chi^{\prime}$ as shown below:

$$
\begin{aligned}
& {[\psi]} \\
& \begin{array}{l}
\frac{\Gamma, F\{x \leftarrow \alpha\} \vdash \Delta}{\Gamma^{\prime}, F\{x \leftarrow \alpha\}, \Pi \vdash \Delta^{\prime}} c: *, w: *{ }^{\left[\delta \backslash \delta^{\prime}\right]} \\
\frac{\Gamma^{\prime \prime}, F\{x \leftarrow \alpha\}, \Pi \vdash \Delta^{\prime \prime}}{} *: *^{[\tau]} \\
\frac{\Gamma^{\prime \prime},(\exists x) F, \Pi \vdash \Delta^{\prime \prime}}{\Gamma^{\prime \prime},(\exists x) F \vdash \Delta^{\prime \prime}} c: l^{[\rho]}\left[\delta^{\left[\delta^{\prime}\right]}\right.
\end{array}
\end{aligned}
$$

(b) $\tau$ is a binary rule and $\rho$ is in the left branch: Then the subproof $\chi$ of $\varphi$ with root $\tau$ has the following form:

$$
\begin{aligned}
& {[\psi]} \\
& \frac{\Gamma, F\{x \leftarrow \alpha\} \vdash \Delta}{\frac{\Gamma,(\exists x) F \vdash \Delta}{\Gamma^{\prime},(\exists x) F \vdash \Delta^{\prime}} c: *, w: *{ }^{[\rho]}} \Gamma_{1}^{\prime} \vdash \Delta_{1}^{\prime} \\
& \left.\frac{\Gamma^{\prime \prime}, \Gamma_{1}^{\prime \prime},(\exists x) F \vdash \Delta^{\prime \prime}, \Delta_{1}^{\prime \prime}}{*}: \tau\right]
\end{aligned}
$$

The left premise of $\tau$ contains $(\exists x) F$ (as a descendant of the main occurrence of $\rho$ ) because neither contractions nor weakenings can remove it. The propositional rule $\tau$ does not have this $(\exists x) F$ as auxiliary formula (for assuming it has would lead to contradiction, because the main formula of $\tau$ wouldn't be in prenex form). Hence the conclusion of $\tau$ also contains this $(\exists x) F$.

Let $\delta^{\prime}$ be the contractions in $\delta$ that are associated with $\rho$ and let $\Pi$ be the multi-set that contains the formula $(\exists x) F$ exactly $n$ times where $n$ is the number of contractions in $\delta^{\prime}$. 
We define $\chi \rightarrow_{M} \chi^{\prime}$ with $\chi^{\prime}$ as shown below:

$$
\begin{gathered}
{[\psi]} \\
\frac{\Gamma, F\{x \leftarrow \alpha\} \vdash \Delta}{\Gamma^{\prime}, F\{x \leftarrow \alpha\}, \Pi \vdash \Delta^{\prime}} c: *, w: *{ }^{\left[\delta \backslash \delta^{\prime}\right]} \quad \Gamma_{1}^{\prime} \vdash \Delta_{1}^{\prime} \\
\frac{\Gamma^{\prime \prime}, \Gamma_{1}^{\prime \prime}, F\{x \leftarrow \alpha\}, \Pi \vdash \Delta^{\prime \prime}, \Delta_{1}^{\prime \prime}}{\frac{\Gamma^{\prime \prime}, \Gamma_{1}^{\prime \prime},(\exists x) F, \Pi \vdash \Delta^{\prime \prime}, \Delta_{1}^{\prime \prime}}{\Gamma^{\prime \prime}, \Gamma_{1}^{\prime \prime},(\exists x) F \vdash \Delta^{\prime \prime}, \Delta_{1}^{\prime \prime}}} \exists::^{[\rho]}{ }^{\left[\delta^{\prime}\right]}
\end{gathered} *^{[\tau]}
$$

(c) $\tau$ is a binary rule and $\rho$ is in the right branch: Then the subproof $\chi$ of $\varphi$ with root $\tau$ has the following form:

$$
\begin{gathered}
{[\psi]} \\
\frac{\Gamma, F\{x \leftarrow \alpha\} \vdash \Delta}{\Gamma,(\exists x) F \vdash \Delta} \exists: l^{[\rho]} \\
\frac{\Gamma_{1}^{\prime} \vdash \Delta_{1}^{\prime}}{\Gamma^{\prime \prime}, \Gamma_{1}^{\prime \prime},(\exists x) F \vdash \Delta^{\prime \prime}, \Delta_{1}^{\prime \prime}} *: \Delta^{\prime} \\
\Gamma^{\prime},(\exists x) F[*]
\end{gathered}
$$

The right premise of $\tau$ contains $(\exists x) F$ (as a descendant of the main occurrence of $\rho$ ) because neither contractions nor weakenings can remove it. The propositional rule $\tau$ does not have this $(\exists x) F$ as auxiliary formula (for assuming it has would lead to contradiction, because the main formula of $\tau$ wouldn't be in prenex form). Hence the conclusion of $\tau$ also contains this $(\exists x) F$.

Let $\delta^{\prime}$ be the contractions in $\delta$ that are associated with $\rho$ and let $\Pi$ be the multi-set that contains the formula $(\exists x) F$ exactly $n$ times where $n$ is the number of contractions in $\delta^{\prime}$.

We define $\chi \rightarrow_{M} \chi^{\prime}$ with $\chi^{\prime}$ as shown below:

$$
\begin{aligned}
& {[\psi]} \\
& \begin{array}{c}
\frac{\Gamma, F\{x \leftarrow \alpha\} \vdash \Delta}{\Gamma_{1}^{\prime} \vdash \Delta_{1}^{\prime} \quad \frac{\Gamma^{\prime}, F\{x \leftarrow \alpha\}, \Pi \vdash \Delta^{\prime}}{\Gamma^{\prime \prime}, \Gamma_{1}^{\prime \prime}, F\{x \leftarrow \alpha\}, \Pi \vdash \Delta^{\prime \prime}, \Delta_{1}^{\prime \prime}}} *: *, w: * *^{\left[\delta \backslash \delta^{\prime}\right]} \\
\frac{\Gamma^{\prime \prime}, \Gamma_{1}^{\prime \prime},(\exists x) F, \Pi \vdash \Delta^{\prime \prime}, \Delta_{1}^{\prime \prime}}{\Gamma^{\prime \prime}, \Gamma_{1}^{\prime \prime},(\exists x) F \vdash \Delta^{\prime \prime}, \Delta_{1}^{\prime \prime}} c: *^{[\rho]} \\
{\left[\delta^{\prime}\right]}
\end{array}
\end{aligned}
$$

The eigen-variable condition of $\rho$ in $\chi^{\prime}$ is fulfilled because $\alpha$ does not occur in $\Gamma, \Delta, \Gamma^{\prime}, \Delta^{\prime}$. In particular, $\alpha$ is not added by a weakening in $\delta$ because the proof is regular.

We define $\varphi \rightarrow_{M} \varphi^{\prime}$ with $\varphi^{\prime} \doteq \varphi_{\nu}\left[\chi^{\prime}\right]$, where $\nu$ is the position of $\chi$ in $\varphi$. In other words, $\rightarrow_{M}$ is compatibility-closed. $\rightarrow_{M}^{*}$ denotes the reflexive and transitive closure of $\rightarrow_{M}$. 
Definition 4.1.4 (Mid-Sequent Normal Form). an LK-Proof $\varphi$ is in midsequent normal form, $M-N F$, if and only if there is no LK-Proof $\varphi^{\prime}$ such that $\varphi \rightarrow_{M} \varphi^{\prime}$.

Theorem 4.1 (Existence of Mid-Sequent Normal Form). For every LKProof $\varphi$, there exists an LK-Proof $\varphi^{\prime}$ such that $\varphi \rightarrow_{M}^{*} \varphi^{\prime}$ and $\varphi^{\prime}$ is in Mid-Sequent Normal Form.

Proof. This follows from Gentzen's Sharpened Hauptsatz (Mid Sequent Theorem), in [6].

Theorem 4.2 (Splitting of a Prenex Proof in Mid-Sequent Normal Form). An LK-Proof in Mid-Sequent Normal Form of a prenex end-sequent and without non-atomic cuts has $\operatorname{degree}(\rho)=0$ for any quantifier-rule $\rho$. This means that there is no propositional or cut-rule below a quantifier-rule. Such a proof can thus be split in two parts: an upper part containing only propositional-rules and structural-rules; and a lower part containing only quantifier-rules, contraction-rules and weakening-rules. However this splitting is not unique, because we can split the proof at any position between the lowest propositional-rule or cut-rule and the highest quantifier-rule. Between these two rules there may be several contractions and weakenings.

Theorem 4.3 (Non-Confluency of Mid-Sequent Reduction). Mid-Sequent Reduction is not confluent. For an LK-Proof $\varphi$, there may be more than one LK-Proof $\varphi^{\prime}$ in mid-sequent normal form such that $\varphi \rightarrow_{M} \varphi^{\prime}$.

Algorithm 4.1 (Herbrand Sequent Extraction via Proof Transformation (Mid-Sequent Reduction)). We may extract a Herbrand sequent from an LK-Proof $\varphi$ with end-sequent in prenex form by executing the following steps:

1. Find (by executing mid-sequent reductions) $\varphi^{\prime}$ such that $\varphi \rightarrow_{M}^{*} \varphi^{\prime}$ and $\varphi^{\prime}$ is in mid-sequent normal form.

2. Let $\rho$ be the lowest axiom-rule, propositional-rule or cut-rule ( $\rho$ in in the path between the end-sequent and any other axiom-rule, propositional-rule or cut-rule). And let $s$ be the conclusion sequent of $\rho$. Then compute and output $H_{M}(\varphi) \doteq \operatorname{set}(U(s))$, where $U(s)$ is the sequent containing only the used occurrences of $s$ and $\operatorname{set}\left(s^{\prime}\right)$ is the set-normalized sequent of $s^{\prime}$ ( $s^{\prime}$ with duplicate occurrences removed). 


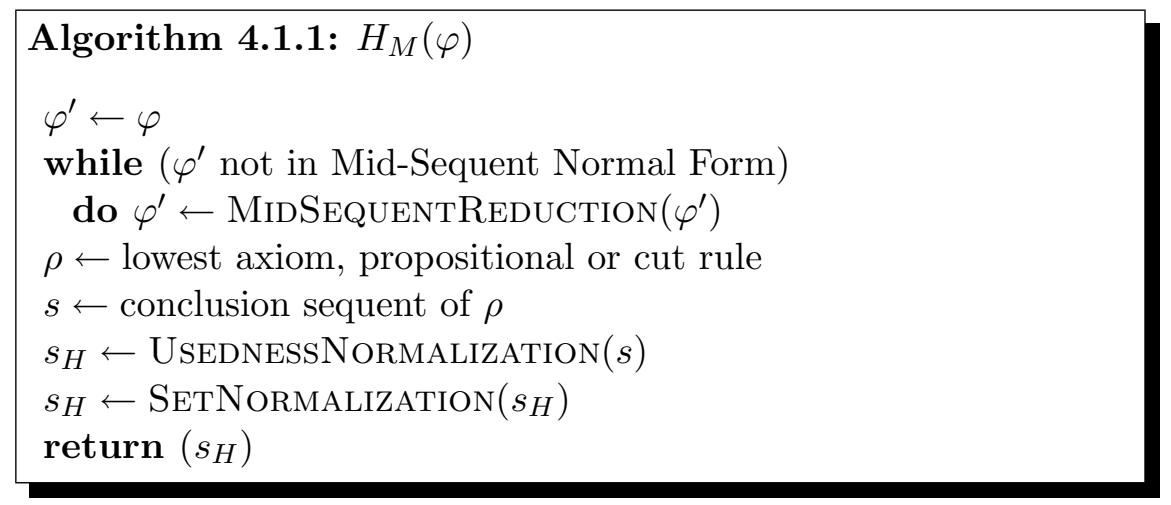

Lemma 4.1 (Extracted Sequent Well-Instantiated). Let $\varphi$ be an LK-Proof and let its end-sequent be $|\varphi|_{[\epsilon]}=A_{1}, \ldots, A_{n} \vdash B_{1}, \ldots, B_{m}$.

$A_{1}^{0}, \ldots, A_{n}^{0}, B_{1}^{0}, \ldots, B_{m}^{0}$ are its formulas without quantifiers. Then the sequent extracted by algorithm 4.1 has the form:

$$
H_{M}(\varphi)=A_{1}^{1}, \ldots, A_{1}^{i_{1}}, \ldots A_{n}^{1}, \ldots, A_{n}^{i_{n}} \vdash B_{1}^{1}, \ldots, B_{1}^{p_{1}}, \ldots, B_{m}^{1}, \ldots, B_{m}^{p_{m}}
$$

where: $A_{j}^{1}, \ldots, A_{j}^{i_{j}}$ are quantifier-free substitution instances of $A_{j}^{0}$ and $B_{k}^{1}, \ldots, B_{k}^{p_{k}}$ are quantifier-free substitution instances of $B_{k}^{0}$.

Proof. Let $\varphi^{\prime}$ and $s$ be a normal form of $\varphi$ and its mid-sequent as specified in algorithm 4.1. Since above $s$ we don't have quantifier-rules, none of the occurrences in $s$ can have quantified variables. Therefore, all occurrences of $s$ are quantifier-free formulas. Furthermore, these occurrences must be substitution instances of the formulas in $|\varphi|_{[\epsilon]}$, because below $s$ we have only quantifier-rules, contractions and weakenings. $H_{M}(\varphi)=\operatorname{set}(U(s))$ is a subsequent of $s$, therefore its occurrences are quantifier-free substitution instances of the occurrences in $|\varphi|_{[\epsilon]}$.

Lemma 4.2 (Validity of the Extracted Sequent). Let $\varphi$ be an LK-Proof. Then $H_{M}(\varphi)$ is valid.

Proof. As specified in algorithm 4.1, let $\varphi^{\prime}$ be a normal form of $\varphi$ and $\rho$ its lowest propositional-rule, axiom-rule or cut-rule. Let $s$ be the conclusion sequent of $\rho$, and let $\nu$ be the position of the subproof of $\varphi^{\prime}$ which has $\rho$ as its root. Then we can construct the following LK-Proof for $H_{M}(\varphi)$, thus demonstrating its validity:

$$
\frac{\left[\left|\varphi^{\prime}\right|_{\nu}\right]}{\overline{H_{M}(\varphi)}} w: *, c: *
$$


Theorem 4.4 (Soundness of Algorithm 4.1). Let $\varphi$ be an LK-Proof. Then the sequent extracted by algorithm $4.1, H_{M}(\varphi)$, is a Herbrand sequent.

Proof. Lemmas 4.1 and 4.2 show that the extracted sequent satisfies the requirements in definition 3.3.2, therefore it is a Herbrand sequent.

Theorem 4.5 (Determinacy of $H_{M}(\varphi)$ ). The output of algorithm $4.1, H_{M}(\varphi)$ is uniquely defined.

Proof. This is not trivial, because the non-confluency of mid-sequent reduction, implying that there may be different proofs $\varphi^{\prime}$ in mid-sequent normal form for $\varphi$, means that the first step of algorithm 4.1 is non-deterministic. However, even though step 1 may result in different proofs $\varphi^{\prime}$, step 2 gives the same result for any of these possible proofs $\varphi^{\prime}$ in mid-sequent normal form. This is a corollary of theorem 4.6, which shows the equality of the results obtained by algorithms 4.1 and 4.2. Since algorithm 4.2 is deterministic, the sequent extracted by algorithm 4.1 must also be uniquely defined.

Definition 4.1.5 (Herbrand Sequent of an LK-Proof in Prenex Form). The determinacy of the output of algorithm 4.1 (as expressed in theorem ??) and theorem 4.8 together allow us to define The Herbrand Sequent of an LKProof $\varphi$ with End-Sequent in Prenex Form, $H_{P}(\varphi)$, as the very result of the algorithm, $H_{M}(\varphi)$.

Example 4.2 (Mid-Sequent Reduction of an LK-Proof with Prenex End-sequent to a $M-N F$ and Extraction of its Herbrand Sequent). Let $\varphi$ be the LK-Proof below:

$$
\begin{gathered}
{\left[\varphi_{*}\right]} \\
\frac{P(0) \vdash P(0) \quad P(s(0)),(\forall x)(P(x) \rightarrow P(s(x))) \vdash P\left(s^{3}(0)\right)}{P(0), P(0) \rightarrow P(s(0)),(\forall x)(P(x) \rightarrow P(s(x))) \vdash P\left(s^{3}(0)\right)} \rightarrow: l \\
\frac{P(0),(\forall x)(P(x) \rightarrow P(s(x))),(\forall x)(P(x) \rightarrow P(s(x))) \vdash P\left(s^{3}(0)\right)}{P(0),(\forall x)(P(x) \rightarrow P(s(x))) \vdash P\left(s^{3}(0)\right)} c: l
\end{gathered} \forall l
$$

where $\varphi_{*}$ is:

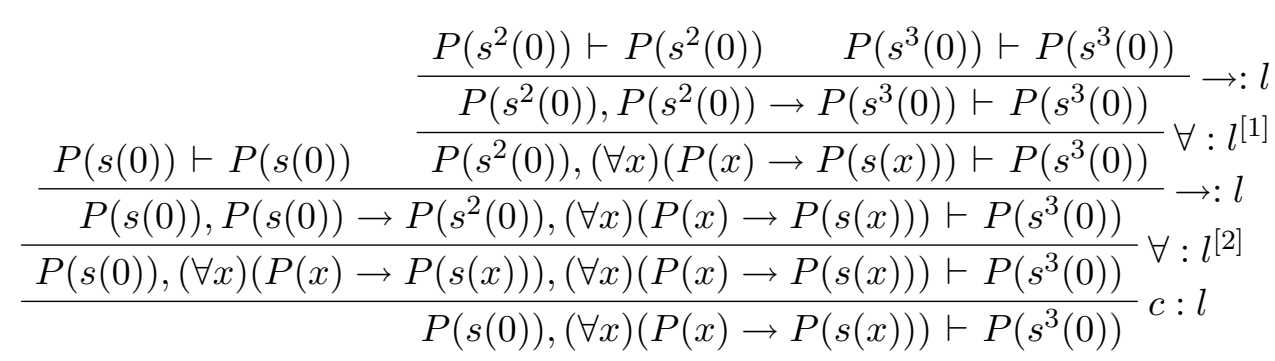


$\varphi$ has two quantifier rules (marked with numbers [1] and [2] that appear above propositional rules. Therefore $\varphi$ is not in $M-N F$. By applying 3 mid-sequent reductions, as demonstrated below, we can produce an $\mathbf{L K}$-Proof $\varphi^{\prime}$ which is in $M-N F$. Firstly, we permute the quantifier rule [1] with the $(\rightarrow:$ l)-rule immediately below it:

$$
\frac{P(s(0)) \vdash P(s(0)) \quad \frac{P\left(s^{2}(0)\right), P\left(s^{2}(0)\right) \rightarrow P\left(s^{3}(0)\right) \vdash P\left(s^{3}(0)\right)}{P\left(s^{2}(0)\right),(\forall x)(P(x) \rightarrow P(s(x))) \vdash P\left(s^{3}(0)\right)}}{P(s(0)), P(s(0)) \rightarrow P\left(s^{2}(0)\right),(\forall x)(P(x) \rightarrow P(s(x))) \vdash P\left(s^{3}(0)\right)} \rightarrow: l
$$

$$
\frac{\frac{P(s(0)) \vdash P(s(0)) \quad P\left(s^{2}(0)\right), P\left(s^{2}(0)\right) \rightarrow P\left(s^{3}(0)\right) \vdash P\left(s^{3}(0)\right)}{P(s(0)), P(s(0)) \rightarrow P\left(s^{2}(0)\right), P\left(s^{2}(0)\right) \rightarrow P\left(s^{3}(0)\right) \vdash P\left(s^{3}(0)\right)} \rightarrow: l}{P(s(0)), P(s(0)) \rightarrow P\left(s^{2}(0)\right),(\forall x)\left(P(x) \rightarrow P(s(x)) \vdash P\left(s^{3}(0)\right)\right.} \forall: l^{[1]}
$$

Secondly, we permute the quantifier rule [2] with the first $(\rightarrow: l)$-rule below it:

$$
\frac{\frac{P(s(0)), P(s(0)) \rightarrow P\left(s^{2}(0)\right),(\forall x)(P(x) \rightarrow P(s(x))) \vdash P\left(s^{3}(0)\right)}{P(0) \vdash P(0) \quad \frac{P(s(0)),(\forall x)(P(x) \rightarrow P(s(x))),(\forall x)(P(x) \rightarrow P(s(x))) \vdash P\left(s^{3}(0)\right)}{P(s(0)),(\forall x)(P(x) \rightarrow P(s(x))) \vdash P\left(s^{3}(0)\right)}}}{P(0), P(0) \rightarrow P(s(0)),(\forall x)(P(x) \rightarrow P(s(x))) \vdash P\left(s^{3}(0)\right)} \rightarrow: l
$$

$l_{M}$

$$
\begin{aligned}
& \frac{P(0) \vdash P(0) \quad P(s(0)), P\left(s(0) \rightarrow P\left(s^{2}(0)\right),(\forall x)(P(x) \rightarrow P(s(x))) \vdash P\left(s^{3}(0)\right)\right.}{P(0), P(0) \rightarrow P(s(0)), P(s(0)) \rightarrow P\left(s^{2}(0)\right),(\forall x)(P(x) \rightarrow P(s(x))) \vdash P\left(s^{3}(0)\right)} \\
& \frac{P(0), P(0) \rightarrow P(s(0)),(\forall x)(P(x) \rightarrow P(s(x))),(\forall x)(P(x) \rightarrow P(s(x))) \vdash P\left(s^{3}(0)\right)}{P(0), P(0) \rightarrow P(s(0)),(\forall x)(P(x) \rightarrow P(s(x))) \vdash P\left(s^{3}(0)\right)} c: l \\
& c: l
\end{aligned}
$$

After these two mid-sequent reductions, our transformed proof is currently: 


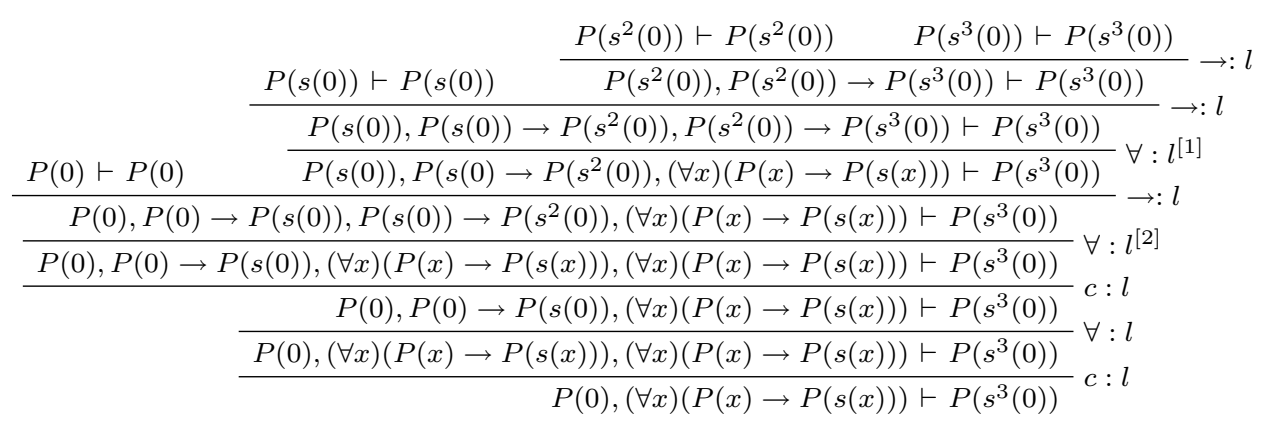

Quantifier rule number [1] is still above propositional rules, therefore another mid-sequent reduction is necessary:

$$
\begin{aligned}
& \frac{P(0) \vdash P(0) \quad \frac{P\left(s(0), P\left(s(0) \rightarrow P\left(s^{2}(0)\right), P\left(s^{2}(0) \rightarrow P\left(s^{3}(0)\right) \vdash P\left(s^{3}(0)\right)\right.\right.\right.}{P\left(s^{2}(0)\right), P\left(s(0) \rightarrow P\left(s^{2}(0)\right),(\forall x)(P(x) \rightarrow P(s(x))) \vdash P\left(s^{3}(0)\right)\right.}}{P(0), P(0) \rightarrow P(s(0)), P(s(0)) \rightarrow P\left(s^{2}(0)\right),(\forall x)(P(x) \rightarrow P(s(x))) \vdash P\left(s^{3}(0)\right)} \rightarrow: l \\
& L_{M} \\
& \frac{\frac{P(0) \vdash P(0) \quad P\left(s(0), P\left(s(0) \rightarrow P\left(s^{2}(0)\right), P\left(s^{2}(0) \rightarrow P\left(s^{3}(0)\right) \vdash P\left(s^{3}(0)\right)\right.\right.\right.}{P(0), P(0) \rightarrow P(s(0)), P(s(0)) \rightarrow P\left(s^{2}(0)\right), P\left(s^{2}(0)\right) \rightarrow P\left(s^{3}(0)\right) \vdash P\left(s^{3}(0)\right)} \rightarrow: l}{P(0), P(0) \rightarrow P(s(0)), P(s(0)) \rightarrow P\left(s^{2}(0)\right),(\forall x)(P(x) \rightarrow P(s(x))) \vdash P\left(s^{3}(0)\right)} \forall: l^{[1]}
\end{aligned}
$$

This last mid-sequent reduction produces an $\mathbf{L K}$-Proof $\varphi^{\prime}$ in $M-N F$ :

$$
\begin{aligned}
& \begin{array}{c}
P(s(0)) \vdash P(s(0)) \quad \frac{P\left(s^{2}(0)\right) \vdash P\left(s^{2}(0)\right) \quad P\left(s^{3}(0)\right) \vdash P\left(s^{3}(0)\right)}{P\left(s^{2}(0)\right), P\left(s^{2}(0)\right) \rightarrow P\left(s^{3}(0)\right) \vdash P\left(s^{3}(0)\right)} \rightarrow: l \\
P(0) \vdash P(0) \quad
\end{array} \rightarrow: l \\
& \frac{P(0), P(0) \rightarrow P(s(0)), P(s(0)) \rightarrow P\left(s^{2}(0)\right), P\left(s^{2}(0)\right) \rightarrow P\left(s^{3}(0)\right) \vdash P\left(s^{3}(0)\right)}{\left.P(0), P(0) \rightarrow P((0)), P((0)) \rightarrow P\left(s^{2}(0)\right),(\forall x)(P(x) \rightarrow P(s))\right) \vdash P\left(s^{3}(0)\right)} \forall: l^{[1]} \\
& P(0), P(0) \rightarrow P(s(0)), P(s(0)) \rightarrow P\left(s^{2}(0)\right),(\forall x)(P(x) \rightarrow P(s(x))) \vdash P\left(s^{3}(0)\right) \quad \forall: l^{[1]} \\
& \frac{P(0), P(0) \rightarrow P(s(0)),(\forall x)(P(x) \rightarrow P(s(x))),(\forall x)(P(x) \rightarrow P(s(x))) \vdash P\left(s^{3}(0)\right)}{\forall: l^{2}} \quad \text { l } \\
& \begin{aligned}
\frac{P(0), P(0) \rightarrow P(s(0)),(\forall x)(P(x) \rightarrow P(s(x))) \vdash P\left(s^{3}(0)\right)}{P(0),(\forall x)(P(x) \rightarrow P(s(x))),(\forall x)(P(x) \rightarrow P(s(x))) \vdash P\left(s^{3}(0)\right)} & \forall: l \\
P(0),(\forall x)(P(x) \rightarrow P(s(x))) \vdash P\left(s^{3}(0)\right) & c: l
\end{aligned}
\end{aligned}
$$

We can now extract the Herbrand sequent of $\varphi$, which in this case is simply the conclusion sequent of the lowest $(\rightarrow: l)$-rule of $\varphi^{\prime}$ :

$$
H_{M}(\varphi)=H_{M}\left(\varphi^{\prime}\right)=\left(\begin{array}{r}
P(0), \\
P(0) \rightarrow P(s(0)), \\
P(s(0)) \rightarrow P\left(s^{2}(0)\right), \\
P\left(s^{2}(0)\right) \rightarrow P\left(s^{3}(0)\right)
\end{array}\right) \vdash P\left(s^{3}(0)\right)
$$




\subsubsection{Extraction via Collection of Instances}

Algorithm 4.1 extracts a Herbrand Sequent by performing several midsequent reductions that transform the original proof. This extensive transformation is quite a waste of computational effort if we are not interested in the transformed proof, but only in a Herbrand Sequent for the end-sequent of the original proof.

It turns out that it is possible to compute the same Herbrand Sequent just by analyzing the original proof, instead of transforming it. In this subsection we explain this algorithm, which was firstly described in [7].

The idea is to notice that quantifier-free substitution instances in occurrences of $H_{P}(\varphi)$ defined in 4.1 .5 are necessarily the auxiliary occurrences of some quantifier-rules in $\varphi$. Therefore we can compute $H_{P}(\varphi)$ just by analyzing $\varphi$, collecting its appropriate auxiliary occurrences and then constructing a sequent by composing the quantifier-free subsequent with the sequent formed from these collected auxiliary occurrences. In other words, we remove the quantified occurrences of the end-sequent and in their place we put their substitution instances given by the auxiliary occurrences of the quantifier-rules.

By showing that the result of applying algorithm 4.2 is exactly the same as the result of applying algorithm 4.1, the soundness of algorithm 4.2 follows trivially from the already proved soundness of algorithm 4.1. Furthermore, since algorithm 4.2 is deterministic, it follows that the non-determinism of algorithm 4.1 is irrelevant, since its result is necessarily unique and equal to the result of algorithm 4.2 .

Definition 4.1.6 (Set of Quantifier Rules). $Q(\varphi)$ is the set of all quantifierrules of the LK-Proof $\varphi$.

Definition 4.1.7 (Sets of Used Auxiliary Occurrences). Let $R$ be a set of rules. With $\mathcal{A}_{A}(R)$ we denote the set of used auxiliary occurrences in the antecedent of the rules in $\mathrm{R}$. With $\mathcal{A}_{C}(R)$ we denote the set of used auxiliary occurrences in the consequent of the rules in $\mathrm{R}$.

Definition 4.1.8 (Used Subsequent). Let $s$ be a sequent. With $U(s)$ we denote the subsequent of $s$ obtained by removing from $s$ all occurrences that are not used.

Definition 4.1.9 (Quantifier-free Subsequent). Let $s$ be a sequent. With $\mathcal{D}(s)$ we denote the subsequent of $s$ obtained by removing from $s$ all occurrences that contain quantifiers.

Algorithm 4.2 (Herbrand Sequent Extraction via Proof Analysis (Instance Collection)). Let $\varphi$ be an LK-Proof with end-sequent $s$ in prenex form. Then a Herbrand sequent of the end-sequent of $\varphi$ may be extracted from $\varphi$ by computing:

$$
H_{I C} \doteq \operatorname{set}\left(s_{P}(\varphi) \circ Q_{P}(\varphi)\right)
$$


where:

$$
\begin{gathered}
s_{P}(\varphi) \doteq \mathcal{D}(U(s)) \\
Q_{P}(\varphi) \doteq \mathcal{D}\left(\operatorname{seq}\left(\mathcal{A}_{A}(Q(\varphi)), \mathcal{A}_{A}(Q(\varphi))\right)\right)
\end{gathered}
$$

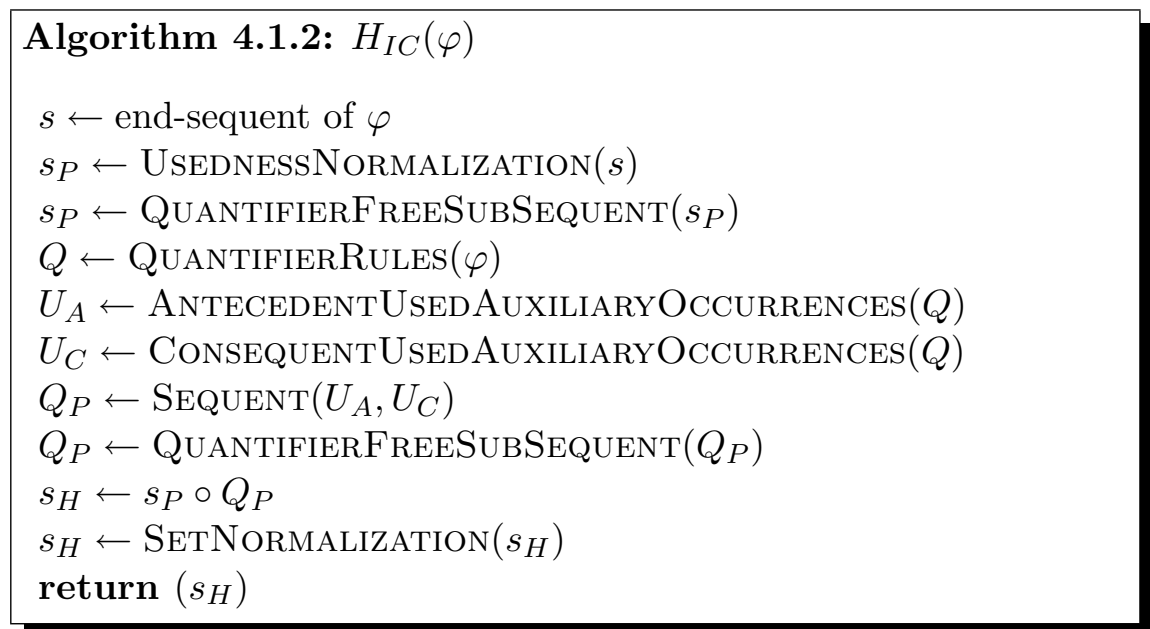

Theorem 4.6 (Equality of Results obtained via Algorithms 4.2 and 4.1). Let $\varphi$ be an LK-Proof with end-sequent in prenex form. Then:

$$
H_{M}(\varphi)=H_{I C}(\varphi)
$$

Proof. The proof below is adapted from [7].

Let $\varphi$ be a prenex LK-Proof. Let $\varphi^{*}$ be a mid-sequent normal form of $\varphi$ computed non-deterministically by algorithm 4.1 .

We proceed by induction on the length $n$ of the Mid-Sequent Reduction sequence of $\varphi$ to $\varphi^{*}$.

1. For $n=0$ we have $\varphi=\varphi^{*}$ and $\varphi$ in Mid-Sequent normal form. We show

$$
H_{M}(\varphi)=\operatorname{set}\left(s_{P}(\varphi) \circ Q_{P}(\varphi)\right)
$$

by induction on the size of the quantifier part.

Let $\rho$ be the lowest propositional-rule, axiom-rule or cut-rule and let $s$ be its conclusion sequent. Then $H_{M}(\varphi)=\operatorname{set}(U(s))$. We can partition $\varphi$ as $\begin{aligned} & \varphi_{p} \\ & \varphi_{q}\end{aligned}$ where $\varphi_{p}$ ends with $\rho$ and $\varphi_{q}$ consists only of quantifier rules, weakenings and contractions (which are all unary). We proceed by induction on $m$, the number of rules in $\varphi_{q}$ :

If $m=0$ then $Q_{P}(\varphi)=\emptyset, s_{P}=U(s)$ and therefore

$$
H_{I C}(\varphi)=\operatorname{set}\left(s_{P}(\varphi) \circ Q_{P}(\varphi)\right)=\operatorname{set}(U(s))=H_{M}(\varphi)
$$


If $m>0$, let $\varphi_{q}=\begin{gathered}\varphi_{q}^{\prime} \\ \rho^{\prime}\end{gathered}$.

By Induction Hypothesis we know for $\varphi^{\prime}=\begin{gathered}\varphi_{p} \\ \varphi_{q}^{\prime}\end{gathered}$ that $H_{M}\left(\varphi^{\prime}\right)=$ $\operatorname{set}\left(s_{P}\left(\varphi^{\prime}\right) \circ Q_{P}\left(\varphi^{\prime}\right)\right)$. But $H_{M}(\varphi)=H_{M}\left(\varphi_{p}\right)=H_{M}\left(\varphi^{\prime}\right)$ and we also have $\operatorname{set}\left(s_{P}\left(\varphi^{\prime}\right) \circ Q_{P}\left(\varphi^{\prime}\right)\right)=\operatorname{set}\left(s_{P}(\varphi) \circ Q_{P}(\varphi)\right)$ because if $\rho^{\prime}$ is a quantifier rule with an unused auxiliary occurrence or a contraction or a weakening, then $\operatorname{set}\left(s_{P}(\varphi)\right)=\operatorname{set}\left(s_{P}\left(\varphi^{\prime}\right)\right)$ and $Q_{P}(\varphi)=Q_{P}\left(\varphi^{\prime}\right)$, and if $\rho^{\prime}$ is a quantifier rule with a used auxiliary formula occurrence $F$, then $F$ moves from $s_{P}\left(\varphi^{\prime}\right)$ into $Q_{P}(\varphi)$.

2. For the induction step $(n>0)$ we show that: if $\varphi \rightarrow_{M} \varphi^{\prime}$, then:

(a) $Q_{P}(\varphi)=Q_{P}\left(\varphi^{\prime}\right)$

(b) $s_{P}(\varphi)=s_{P}\left(\varphi^{\prime}\right)$.

That $s_{P}(\varphi)=s_{P}\left(\varphi^{\prime}\right)$ is the case comes from the fact that mid-sequent reduction does not modify the end-sequent and preserves usedness.

To prove that $Q_{P}(\varphi)=Q_{P}\left(\varphi^{\prime}\right)$ we observe that no quantifier rules are added, nor removed. Moreover, mid-sequent reduction also does not add nor remove weakenings and the ancestor relation in the modified parts is not modified. So

$$
\operatorname{seq}\left(\mathcal{A}_{A}(Q(\varphi)), \mathcal{A}_{C}(Q(\varphi))\right)=\operatorname{seq}\left(\mathcal{A}_{A}\left(Q\left(\varphi^{\prime}\right)\right), \mathcal{A}_{C}\left(Q\left(\varphi^{\prime}\right)\right)\right)
$$

, and thus $Q_{P}(\varphi)=Q_{P}\left(\varphi^{\prime}\right)$.

Lemma 4.3 (Extracted Sequent Well-Instantiated). Let $\varphi$ be an LK-Proof and let its end-sequent be $|\varphi|_{[\epsilon]}=A_{1}, \ldots, A_{n} \vdash B_{1}, \ldots, B_{m} . A_{1}^{0}, \ldots, A_{n}^{0}, B_{1}^{0}, \ldots, B_{m}^{0}$ are its formulas without quantifiers. Then the sequent extracted by algorithm 4.2 has the form:

$$
H_{I C}(\varphi)=A_{1}^{1}, \ldots, A_{1}^{i_{1}}, \ldots A_{n}^{1}, \ldots, A_{n}^{i_{n}} \vdash B_{1}^{1}, \ldots, B_{1}^{p_{1}}, \ldots, B_{m}^{1}, \ldots, B_{m}^{p_{m}}
$$

where: $A_{j}^{1}, \ldots, A_{j}^{i_{j}}$ are quantifier-free substitution instances of $A_{j}^{0}$ and $B_{k}^{1}, \ldots, B_{k}^{p_{k}}$ are quantifier-free substitution instances of $B_{k}^{0}$.

Proof. By theorem 4.6 we have $H_{I C}(\varphi)=H_{M}(\varphi)$, and by lemma 4.1, $H_{M}(\varphi)$ has the desired form. Therefore $H_{I C}(\varphi)$ also has the desired form.

Lemma 4.4 (Validity of the Extracted Sequent). Let $\varphi$ be an LK-Proof. Then $H_{I} C(\varphi)$ is valid. 
Proof. By theorem 4.6 we have $H_{I C}(\varphi)=H_{M}(\varphi)$, and by lemma 4.2, $H_{M}(\varphi)$ is valid. Therefore $H_{I C}(\varphi)$ is also valid.

$$
\frac{\left[\left|\varphi^{\prime}\right|_{\nu}\right]}{\overline{H_{M}(\varphi)}} w: *, c: *
$$

Theorem 4.7 (Soundness of Algorithm 4.2). Let $\varphi$ be an LK-Proof. Then the sequent extracted by algorithm $4.2, H_{I} C(\varphi)$, is a Herbrand sequent.

Proof. Lemmas 4.3 and 4.4 show that the extracted sequent satisfies the requirements in definition 3.3.2, therefore it is a Herbrand sequent.

Example 4.3 (Extraction of the Herbrand Sequent of an LK-Proof of a Prenex End-Sequent without Proof Transformation). Let $\varphi$ be the LK-Proof in example 4.2:

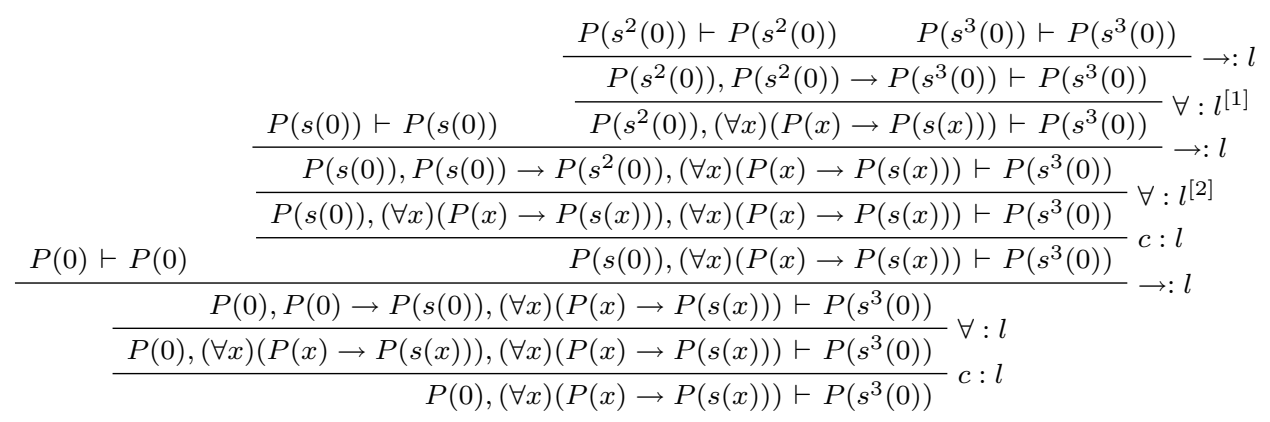

The set $Q(\varphi)$ of quantifier rules of $\varphi$ has the following rules:

$$
\begin{gathered}
\frac{P\left(s^{2}(0)\right), P\left(s^{2}(0)\right) \rightarrow P\left(s^{3}(0)\right) \vdash P\left(s^{3}(0)\right)}{P\left(s^{2}(0)\right),(\forall x)(P(x) \rightarrow P(s(x))) \vdash P\left(s^{3}(0)\right)} \forall: l^{[1]} \\
\frac{P(s(0)), P(s(0)) \rightarrow P\left(s^{2}(0)\right),(\forall x)(P(x) \rightarrow P(s(x))) \vdash P\left(s^{3}(0)\right)}{P(s(0)),(\forall x)(P(x) \rightarrow P(s(x))),(\forall x)(P(x) \rightarrow P(s(x))) \vdash P\left(s^{3}(0)\right)} \forall: l^{[2]} \\
\frac{P(0), P(0) \rightarrow P(s(0)),(\forall x)(P(x) \rightarrow P(s(x))) \vdash P\left(s^{3}(0)\right)}{P(0),(\forall x)(P(x) \rightarrow P(s(x))),(\forall x)(P(x) \rightarrow P(s(x))) \vdash P\left(s^{3}(0)\right)} \forall: l
\end{gathered}
$$

Its set of used auxiliary antecedent occurrences is:

$$
\mathcal{A}_{A}(Q(\varphi))=\left\{\begin{array}{l}
P(0) \rightarrow P(s(0)) \\
P(s(0)) \rightarrow P\left(s^{2}(0)\right), \\
P\left(s^{2}(0)\right) \rightarrow P\left(s^{3}(0)\right)
\end{array}\right\}
$$

Its set of used auxiliary consequent occurrences is:

$$
\mathcal{A}_{C}(Q(\varphi))=\{\}
$$


The sequent formed from these used occurrences is:

$$
\left.\left.\operatorname{seq}\left(\mathcal{A}_{A}(Q(\varphi))\right), \mathcal{A}_{C}(Q(\varphi))\right)\right)=\left(\begin{array}{c}
P(0) \rightarrow P(s(0)) \\
P(s(0)) \rightarrow P\left(s^{2}(0)\right) \\
P\left(s^{2}(0)\right) \rightarrow P\left(s^{3}(0)\right)
\end{array}\right) \vdash
$$

Since all the formulas in $\left.\left.\operatorname{seq}\left(\mathcal{A}_{A}(Q(\varphi))\right), \mathcal{A}_{C}(Q(\varphi))\right)\right)$ are quantifier-free, we have:

$$
\begin{aligned}
Q_{P}(\varphi) & \left.\left.=\mathcal{D}\left(\operatorname{seq}\left(\mathcal{A}_{A}(Q(\varphi))\right), \mathcal{A}_{C}(Q(\varphi))\right)\right)\right) \\
& \left.\left.=\operatorname{seq}\left(\mathcal{A}_{A}(Q(\varphi))\right), \mathcal{A}_{C}(Q(\varphi))\right)\right) \\
& =\left(\begin{array}{c}
P(0) \rightarrow P(s(0)) \\
P(s(0)) \rightarrow P\left(s^{2}(0)\right) \\
P\left(s^{2}(0)\right) \rightarrow P\left(s^{3}(0)\right)
\end{array}\right) \vdash
\end{aligned}
$$

Furthermore:

$$
\begin{aligned}
s_{P}(\varphi) & =\mathcal{D}(U(s)) \\
& =P(0) \vdash P\left(s^{3}(0)\right)
\end{aligned}
$$

And hence, we have:

$$
\begin{aligned}
H(\varphi) & =\operatorname{set}\left(s_{P}(\varphi) \circ Q_{P}(\varphi)\right) \\
& =\left(\begin{array}{r}
P(0), \\
P(0) \rightarrow P(s(0)), \\
P(s(0)) \rightarrow P\left(s^{2}(0)\right), \\
P\left(s^{2}(0)\right) \rightarrow P\left(s^{3}(0)\right.
\end{array}\right) \vdash P\left(s^{3}(0)\right)
\end{aligned}
$$




\subsection{Extraction from Non-Prenex Proofs}

When the end-sequent is not in Prenex form, mid-sequent reduction clearly cannot be used to extract a Herbrand sequent, because rules referring to quantifiers nested under propositional connectives must necessarily occur above the rules referring to these propositional connectives, and thus midsequent reduction will not be capable of pushing down these quantifier rules until they are all below all the propositional rules. Example 4.4 shows this.

Algorithm 4.2 also cannot be used, because the collected instances are all included without distinction with depth 0 in the constructed Herbrand sequent, while in the Non-Prenex case, these instances should be included in the nested position from which they originate.

In principle, those two algorithms could still be used, if the non-prenex LK-Proofs were firstly transformed to prenex form, but this would decrease human-understandability, because it is easier for humans to understand formulas and proofs when the quantifiers are in their original places than in the beginning of the formulas as a result of prenexification.

Therefore, we must design new algorithms to extract Herbrand sequents from LK-Proofs of end-sequents in non-prenex form. Here we describe two such algorithms. The first one, Extraction via Proof Transformation to Quantifier-free $\mathbf{L} \mathbf{K}_{\mathbf{A}}$, was described in [1] and involves the definition of an auxiliary formula-structure (array-formula) and modifications in the Sequent Calculus to deal with this structure. The second algorithm, Extraction via Collection of Sub-Formula Instances, generalizes the algorithm of Extraction via Collection of Instances for the case of non-prenex end-sequents by using the same formula-structure defined for the first algorithm.

Example 4.4 (End-sequent in non-prenex form and an LK-Proof for it). Let the end-sequent be $P(0) \wedge(\forall x)\left(P(x) \rightarrow P(s(x)) \vdash P\left(s^{2}(0)\right)\right.$, which is not in prenex-form. Any LK-proof of this end-sequent will have a propositional rule $\wedge: l$ below the quantifier rules $\forall: l$, which are responsible for inserting the $\forall$-quantifier in copies (obtained by contraction) of the sub-formula $\forall x(P(x) \rightarrow P(s(x))$. One example of such a proof is the LK-Proof $\varphi$ shown below. A Herbrand sequent cannot be obtained by applying the mid-sequent theorem in this case, because the quantifier rules cannot be pushed down until they are brought under the $\wedge: l$ rule.

$$
\begin{gathered}
{\left[\varphi^{\prime}\right]} \\
\frac{\frac{P(0), P(0) \rightarrow P(s(0)), P(s(0)) \rightarrow P\left(s^{2}(0)\right) \vdash P\left(s^{2}(0)\right)}{P(0), P(0) \rightarrow P(s(0)),(\forall x)\left(P(x) \rightarrow P(s(x)) \vdash P\left(s^{2}(0)\right)\right.}}{P(0), \forall x\left(P(x) \rightarrow P(s(x)),(\forall x)\left(P(x) \rightarrow P(s(x)) \vdash P\left(s^{2}(0)\right)\right.\right.} \forall: l \\
\frac{P(0), \forall x\left(P(x) \rightarrow P(s(x)) \vdash P\left(s^{2}(0)\right)\right.}{P(0) \wedge(\forall x)\left(P(x) \rightarrow P(s(x)) \vdash P\left(s^{2}(0)\right)\right.} \wedge: l
\end{gathered}
$$




\subsubsection{Extraction via Proof Transformation to Quantifier-free $\mathbf{L K}_{\mathrm{A}}$}

The algorithm described in this subsection is restricted to LK-Proofs of end-sequents with weak quantifiers only. The reason for this is that the proof transformations used in this algorithm would violate the eigen-variable conditions. This is not a limitation for the algorithm, since end-sequents may have their strong quantifiers eliminated via skolemization. Therefore, unless stated otherwise, we assume that sequents don't have strong quantifiers.

The method basically consists of transforming the LK-Proof into a quantifier free proof in a modified version of Sequent Calculus called $\mathbf{L} \mathbf{K}_{\mathbf{A}}$, which admits sequents containing so-called array-formulas. Then the end-sequent of this $\mathbf{L K}_{\mathbf{A}}$-Proof is transformed back to a sequent without array-formulas. As proved in theorem 4.8, this final sequent is indeed a Herbrand sequent of the end-sequent of the original LK-Proof, and thus the algorithm here described is sound. Furthermore, the soundness and determinism of the algorithm allow us to associate a unique Herbrand sequent for each $L K-$ Proof of an end-sequent in non-prenex form with weak quantifiers only (Definition 4.2.4).

Definition 4.2.1 (Array Formula). An array formula is defined inductively as:

1. First-Order-Logic Formulas are Array Formulas

2. If $A_{1}, A_{2}, \ldots, A_{n}$ are Array Formulas, then $\left\langle A_{1}, A_{2}, \ldots, A_{n}\right\rangle$

3. If $A, B$ are Array Formulas, then $\neg A, A \vee B, A \wedge B, A \rightarrow B$ are Array Formulas.

Definition 4.2.2 (Sequent Calculus $\mathbf{L} \mathbf{K}_{\mathbf{A}}$ ). If $A_{1}, A_{2}, \ldots, A_{n}, B_{1}, B_{2}, \ldots, B_{m}$ are Array Formulas, then $A_{1}, A_{2}, \ldots, A_{n}, \vdash B_{1}, B_{2}, \ldots, B_{m}$ is called an Array Sequent.

The Sequent Calculus $\mathbf{L K}_{\mathbf{A}}$ is the Sequent Calculus $\mathbf{L K}$ with addition of the following rules:

$$
\frac{\Delta, A, \Gamma, B, \Pi \vdash \Lambda}{\Delta,\langle A, B\rangle, \Gamma, \Pi \vdash \Lambda}\langle\rangle: l \quad \frac{\Lambda \vdash \Delta, A, \Gamma, B, \Pi}{\Lambda \vdash \Delta,\langle A, B\rangle, \Gamma, \Pi}\langle\rangle: l
$$

Algorithm 4.3 ( $\Psi$ : Transformation of LK-Proofs into Quantifier-free $\mathbf{L K}_{\mathbf{A}}$-Proofs). We define a mapping $\Psi$ which transforms an arbitrary $\mathbf{L K}$-Proof $\varphi$ into an $\mathbf{L K}_{\mathbf{A}}$-Proof without quantifiers by executing the following two steps:

1. We construct $\varphi^{\prime}$ by omitting all quantifier-rules. 
2. $\varphi^{\prime}$ is generally not an LK-Proof, because its remaining contractionrules are not necessarily correct anymore. Therefore we replace these "false" contraction-rules (starting from above downwards) by \langle\rangle$: l$ and \langle\rangle$: r$ rules. If

$$
\frac{\Gamma, A_{1}, \Delta, A_{2}, \Pi \vdash \Lambda}{\Gamma, A_{3}, \Delta, \Pi \vdash \Lambda} c: l
$$

occurs in $\varphi^{\prime}$ and $A_{1} \neq A_{2}$, then replace it by:

$$
\frac{\Gamma, A_{1}, \Delta, A_{2}, \Pi \vdash \Lambda}{\Gamma,\left\langle A_{1}, A_{2}\right\rangle, \Delta, \Pi \vdash \Lambda}\langle\rangle: l
$$

Analogously, if:

$$
\frac{\Lambda \vdash \Gamma, A_{1}, \Delta, A_{2}, \Pi}{\Lambda \vdash \Gamma, A_{3}, \Delta, \Pi} c: r
$$

occurs in $\varphi^{\prime}$ and $A_{1} \neq A_{2}$, then replace it by:

$$
\frac{\Lambda \vdash \Gamma, A_{1}, \Delta, A_{2}, \Pi}{\Lambda \vdash \Gamma,\left\langle A_{1}, A_{2}\right\rangle, \Delta, \Pi}\langle\rangle: r
$$

$\Psi(\varphi)$ is the quantifier-free $\mathbf{L K}_{\mathbf{A}}$-Proof obtained by executing the two steps above.

Example 4.5 (Transforming an LK-Proof with quantifiers into a quantifier-free $\mathbf{L K}_{\mathbf{A}}$-proof). Let $\varphi$ be the $\mathbf{L K}$-proof shown in example 4.4. Firstly we omit all quantifier rules and obtain the following $\mathbf{L K}$-Proof $\varphi_{c}$ with false contractions:

$$
\frac{\left[\varphi^{\prime}\right]}{\frac{P(0), P(0) \rightarrow P(s(0)), P(s(0)) \rightarrow P\left(s^{2}(0)\right) \vdash P\left(s^{2}(0)\right)}{P(0),(\forall x)\left(P(x) \rightarrow P(s(x)) \vdash P\left(s^{2}(0)\right)\right.}} c: l
$$

Then we replace the false contraction rule by an array formation rule, thus obtaining the $\mathbf{L K}_{\mathbf{A}}$-Proof $\Psi(\varphi)$.

$$
\begin{aligned}
& {\left[\varphi^{\prime}\right]} \\
& \frac{\frac{P(0), P(0) \rightarrow P(s(0)), P(s(0)) \rightarrow P\left(s^{2}(0)\right) \vdash P\left(s^{2}(0)\right)}{P(0),\left\langle P(0) \rightarrow P(s(0)), P(s(0)) \rightarrow P\left(s^{2}(0)\right)\right\rangle \vdash P\left(s^{2}(0)\right)}}{P(0) \wedge\left\langle P(0) \rightarrow P(s(0)), P(s(0)) \rightarrow P\left(s^{2}(0)\right)\right\rangle \vdash P\left(s^{2}(0)\right)} \wedge: l
\end{aligned}
$$

Definition 4.2.3 ( $\Phi$ : Mapping from Array Formulas to sequences of FOLFormulas). We define a mapping $\Phi$ which transforms array formulas and sequents into First-Order Logic formulas and sequents. In other words, $\Phi$ eliminates $\langle\ldots\rangle$ and can be defined inductively by: 
1. If $A \in F_{F} O L$, then $\Phi(A) \doteq A$

2. $\Phi(\langle A, B\rangle) \doteq \Phi(A), \Phi(B)$

3. If $\Phi(A)=A_{1}, \ldots, A_{n}$, then $\Phi(\neg A) \doteq \neg A_{1}, \ldots, \neg A_{n}$

4. If $\Phi(A)=A_{1}, \ldots, A_{n}$ and $\Phi(B)=B_{1}, \ldots, B_{m}$, then $\Phi(A \circ B) \doteq$ $A_{1} \circ B_{1}, \ldots, A_{1} \circ B_{m}, \ldots, A_{n} \circ B_{1}, \ldots, A_{n} \circ B_{m}$, for $\circ \in\{\wedge, \vee, \rightarrow\}$

5. If $\Phi(A)=A_{1}, \ldots, A_{n}$, then $\Phi((Q x)(A)) \doteq(Q x)\left(A_{1}\right), \ldots,(Q x)\left(A_{n}\right)$, for $Q \in\{\forall, \exists\}$.

6. $\Phi\left(A_{1}, \ldots, A_{n} \vdash B_{1}, \ldots, B_{m}\right) \doteq \Phi\left(A_{1}\right), \ldots, \Phi\left(A_{n}\right) \vdash \Phi\left(B_{1}\right), \ldots, \Phi\left(B_{m}\right)$

Example 4.6 (Mapping from Array Formulas to sequences of FOL-Formulas). $\operatorname{Let} \Psi(\varphi)$ be the $\mathbf{L K}_{\mathbf{A}}$-Proof in example 4.5. Then, its end-sequent mapped to FOL-Formulas is:

$$
\Phi\left(|\Psi(\varphi)|_{[\epsilon]}\right)=\left(\begin{array}{c}
P(0) \wedge P(0) \rightarrow P(s(0)), \\
P(0) \wedge P(s(0)) \rightarrow P\left(s^{2}(0)\right)
\end{array}\right) \vdash P\left(s^{2}(0)\right)
$$

Algorithm 4.4 (Herbrand Sequent Extraction via Proof Transformation to Quantifier-free $\left.\mathbf{L K}_{\mathbf{A}}\right)$. Let $\varphi$ be an LK-Proof. A Herbrand Sequent of $|\varphi|_{[\epsilon]}$ can be obtained by computing:

$$
H_{L K_{A}}(\varphi) \doteq \Phi\left(|\Psi(\varphi)|_{[\epsilon]}\right)
$$

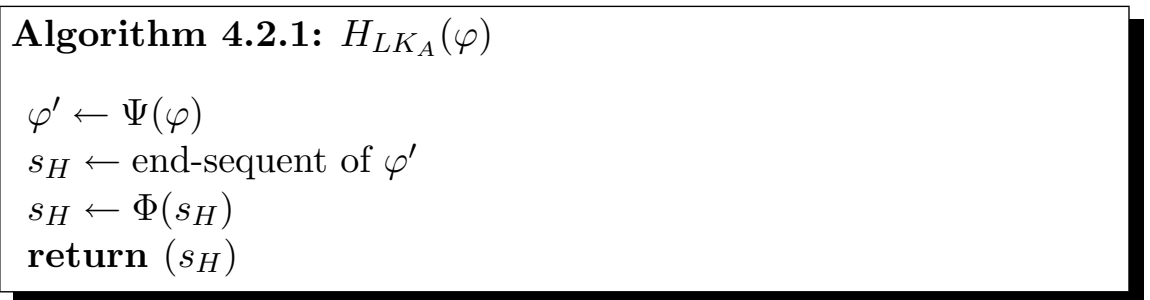

Lemma 4.5 (Extracted Sequent Well-Instantiated). Let $\varphi$ be an LK-Proof and let its end-sequent be $|\varphi|_{[\epsilon]}=A_{1}, \ldots, A_{n} \vdash B_{1}, \ldots, B_{m}$. $A_{1}^{0}, \ldots, A_{n}^{0}, B_{1}^{0}, \ldots, B_{m}^{0}$ are its formulas without quantifiers. Then the sequent extracted by algorithm 4.4 has the form:

$$
H_{L K_{A}}(\varphi)=A_{1}^{1}, \ldots, A_{1}^{i_{1}}, \ldots A_{n}^{1}, \ldots, A_{n}^{i_{n}} \vdash B_{1}^{1}, \ldots, B_{1}^{p_{1}}, \ldots, B_{m}^{1}, \ldots, B_{m}^{p_{m}}
$$

where: $A_{j}^{1}, \ldots, A_{j}^{i_{j}}$ are quantifier-free substitution instances of $A_{j}^{0}$ and $B_{k}^{1}, \ldots, B_{k}^{p_{k}}$ are quantifier-free substitution instances of $B_{k}^{0}$.

Proof. By definition of $\Phi([1])$. 
Algorithm 4.5 ( $\Phi_{P}$ : Transformation of an $\mathbf{L K}_{\mathbf{A}}$-Proof into an $\mathbf{L K}$-Proof). We define a mapping $\Phi_{P}$ which transforms an arbitrary $\mathbf{L K}_{\mathbf{A}}$-Proof $\varphi$ into an LK-Proof without quantifiers by replacing (starting from above downwards) the \langle\rangle$: l$ and \langle\rangle$: r$ rules. If

$$
\frac{\Gamma, A_{1}, \Delta, A_{2}, \Pi \vdash \Lambda}{\Gamma,\left\langle A_{1}, A_{2}\right\rangle, \Delta, \Pi \vdash \Lambda}\langle\rangle: l
$$

occurs in $\varphi^{\prime}$, then replace it by:

$$
\frac{\Gamma, A_{1}, \Delta, A_{2}, \Pi \vdash \Lambda}{\Gamma,\left(A_{1} \wedge A_{2}\right), \Delta, \Pi \vdash \Lambda} \wedge: l
$$

Analogously, if:

$$
\frac{\Lambda \vdash \Gamma, A_{1}, \Delta, A_{2}, \Pi}{\Lambda \vdash \Gamma,\left\langle A_{1}, A_{2}\right\rangle, \Delta, \Pi}\langle\rangle: r
$$

occurs in $\varphi^{\prime}$ and $A_{1} \neq A_{2}$, then replace it by:

$$
\frac{\Lambda \vdash \Gamma, A_{1}, \Delta, A_{2}, \Pi}{\Lambda \vdash \Gamma,\left(A_{1} \vee A_{2}\right), \Delta, \Pi} \vee: r
$$

$\Phi_{P}(\varphi)$ is the quantifier LK-Proof obtained by executing the procedure above.

Lemma 4.6 (Equivalence of $H_{L K_{A}}(\varphi)$ and $\left.\left|\Phi_{P}(\Psi(\varphi))\right|_{[\epsilon]}\right)$. Let $\varphi$ be an LKProof. Then the sequents $H_{L K_{A}}(\varphi)$ and $\left|\Phi_{P}(\Psi(\varphi))\right|_{[\epsilon]}$ are logically equivalent.

Proof. Let $s \doteq|\varphi|_{[\varepsilon]}$ be the end-sequent of $\varphi$. Let $s^{\prime} \doteq|\Psi(\varphi)|_{[\varepsilon]}$ be the endsequent of $\Psi(\varphi)$. Let $s^{*} \doteq\left|\Phi_{P}(\Psi(\varphi))\right|_{[\varepsilon]}$ be the end-sequent of $\Phi_{P}(\Psi(\varphi))$. By the definition of $\Phi_{P}$, we note that:

- If $\left\langle A_{1}, A_{2}\right\rangle$ occurs positively in $s^{\prime}$, then $\left(A_{1} \vee A_{2}\right)$ occurs in the corresponding position in $s^{*}$;

- If $\left\langle A_{1}, A_{2}\right\rangle$ occurs negatively in $s^{\prime}$, then $\left(A_{1} \wedge A_{2}\right)$ occurs in the corresponding position in $s^{*}$;

Now we prove, by structural induction, that for each formula $A$ in position $\mu$ in $s^{\prime}$, the sequence of formulas $\Phi(A)$ is logically equivalent to $\left|s^{*}\right|_{\mu}$ :

- If $A \in F_{F O L}$, then $\Phi(A)=A=\left|s^{*}\right|_{\mu}$.

- If $A=\left\langle A_{1}, A_{2}\right\rangle$ occurs positively, then $\left|s^{*}\right|_{\mu}=\left(A_{1} \vee A_{2}\right)$. Furthermore $s^{*}=\Gamma \vdash \Delta,\left(A_{1} \vee A_{2}\right), \Pi \sim \Gamma \vdash \Delta, A_{1}, A_{2}, \Pi$. Hence $\left|s^{*}\right|_{\mu} \sim A_{1}^{+}, A_{2}^{+}=$ $\Phi(A)$. 
- If $A=\left\langle A_{1}, A_{2}\right\rangle$ occurs negatively, then $\left|s^{*}\right|_{\mu}=\left(A_{1} \wedge A_{2}\right)$. Furthermore $s^{*}=\Pi,\left(A_{1} \wedge A_{2}\right), \Gamma \vdash \Delta \sim \Pi, A_{1}, A_{2}, \Gamma \vdash \Delta$. Hence $\left|s^{*}\right|_{\mu} \sim A_{1}^{-}, A_{2}^{-}=$ $\Phi(A)$.

- If $A$ is of the form $\neg B$ and $B$ occurs positively and $\Phi(B)=B_{1}, \ldots, B_{n}$, then by induction hypothesis, we have $\left|s^{*}\right|_{(\mu, 1)} \sim \Phi(B)^{+} \sim\left(\left(\ldots\left(B_{1} \vee\right.\right.\right.$ $\left.\left.\left.B_{2}\right) \ldots\right) \vee B_{n}\right)$. Hence $\left|s^{*}\right|_{\mu} \sim\left(\left(\ldots\left(\neg B_{1} \wedge \neg B_{2}\right) \ldots\right) \wedge \neg B_{n}\right)$. And since $\Phi(A)=\neg B_{1}, \ldots, \neg B_{n}$, we have: $\left|s^{*}\right|_{\mu} \sim \Phi(A)^{-}$.

- If $A$ is of the form $\neg B$ and $B$ occurs negatively and $\Phi(B)=B_{1}, \ldots, B_{n}$, then by induction hypothesis, we have $\left|s^{*}\right|_{(\mu, 1)} \sim \Phi(B)^{-} \sim\left(\left(\ldots\left(B_{1} \wedge\right.\right.\right.$ $\left.\left.\left.B_{2}\right) \ldots\right) \wedge B_{n}\right)$. Hence $\left|s^{*}\right|_{\mu} \sim\left(\left(\ldots\left(\neg B_{1} \vee \neg B_{2}\right) \ldots\right) \vee \neg B_{n}\right)$. And since $\Phi(A)=\neg B_{1}, \ldots, \neg B_{n}$, we have: $\left|s^{*}\right|_{\mu} \sim \Phi(A)^{+}$.

- If $A$ occurs positively and it is of the form $B \circ C$ with $\circ \in\{\wedge, \vee\}$ and $\Phi(B)=B_{1}, \ldots, B_{n}$ and $\Phi(C)=C_{1}, \ldots, C_{m}$, then by induction hypothesis we have $\left|s^{*}\right|_{(\mu, 1)} \sim \Phi(B)^{+} \sim\left(\left(\ldots\left(B_{1} \vee B_{2}\right) \ldots \vee B_{n}\right)\right.$ and $\left|s^{*}\right|_{(\mu, 2)} \sim \Phi(C)^{+} \sim\left(\left(\ldots\left(C_{1} \vee C_{2}\right) \ldots \vee C_{n}\right)\right.$. Hence, by distributivity of $\circ$ with respect to $\vee,\left|s^{*}\right|_{\mu} \sim\left(\left(\ldots\left(\left(\ldots\left(B_{1} \circ C_{1}\right) \vee \ldots\right) \vee\left(B_{1} \circ C_{m}\right)\right) \ldots \vee\right.\right.$ $\left(\left(\ldots\left(B_{n} \circ C_{1}\right) \vee \ldots\right) \vee\left(B_{n} \circ C_{m}\right)\right)$. Therefore, $\left|s^{*}\right|_{\mu} \sim \Phi(A)^{+}=B_{1} \circ$ $C_{1}, \ldots, B_{1} \circ C_{m}, \ldots, B_{n} \circ C_{1}, \ldots, B_{n} \circ C_{m}$.

- If $A$ occurs negatively and it is of the form $B \circ C$ with $\circ \in\{\wedge, \vee\}$ and $\Phi(B)=B_{1}, \ldots, B_{n}$ and $\Phi(C)=C_{1}, \ldots, C_{m}$, then by induction hypothesis we have $\left|s^{*}\right|_{(\mu, 1)} \sim \Phi(B)^{-} \sim\left(\left(\ldots\left(B_{1} \wedge B_{2}\right) \ldots \wedge B_{n}\right)\right.$ and $\left|s^{*}\right|_{(\mu, 2)} \sim \Phi(C)^{-} \sim\left(\left(\ldots\left(C_{1} \wedge C_{2}\right) \ldots \wedge C_{n}\right)\right.$. Hence, by distributivity of $\circ$ with respect to $\wedge,\left|s^{*}\right|_{\mu} \sim\left(\left(\ldots\left(\left(\ldots\left(B_{1} \circ C_{1}\right) \wedge \ldots\right) \wedge\left(B_{1} \circ C_{m}\right)\right) \ldots \wedge\right.\right.$ $\left(\left(\ldots\left(B_{n} \circ C_{1}\right) \wedge \ldots\right) \wedge\left(B_{n} \circ C_{m}\right)\right)$. Therefore, $\left|s^{*}\right|_{\mu} \sim \Phi(A)^{-}=B_{1} \circ$ $C_{1}, \ldots, B_{1} \circ C_{m}, \ldots, B_{n} \circ C_{1}, \ldots, B_{n} \circ C_{m}$.

- If $A$ occurs positively and it is of the form $B \rightarrow C$, and $\Phi(B)=$ $B_{1}, \ldots, B_{n}$ and $\Phi(C)=C_{1}, \ldots, C_{m}$, then by induction hypothesis we have $\left|s^{*}\right|_{(\mu, 1)} \sim \Phi(B)^{-} \sim\left(\left(\ldots\left(B_{1} \wedge B_{2}\right) \ldots \wedge B_{n}\right)\right.$ and $\left|s^{*}\right|_{(\mu, 2)} \sim$ $\Phi(C)^{+} \sim\left(\left(\ldots\left(C_{1} \vee C_{2}\right) \ldots \vee C_{n}\right)\right.$. Hence, by distributivity of $\rightarrow$ with respect to $\vee$ and $\wedge,\left|s^{*}\right|_{\mu} \sim\left(\left(\ldots\left(\left(\ldots\left(B_{1} \rightarrow C_{1}\right) \vee \ldots\right) \vee\left(B_{1} \rightarrow\right.\right.\right.\right.$ $\left.\left.C_{m}\right)\right) \ldots \vee\left(\left(\ldots\left(B_{n} \rightarrow C_{1}\right) \vee \ldots\right) \vee\left(B_{n} \rightarrow C_{m}\right)\right)$. Therefore, $\left|s^{*}\right|_{\mu} \sim$ $\Phi(A)^{+}=B_{1} \rightarrow C_{1}, \ldots, B_{1} \rightarrow C_{m}, \ldots, B_{n} \rightarrow C_{1}, \ldots, B_{n} \rightarrow C_{m}$.

- If $A$ occurs negatively and it is of the form $B \rightarrow C$, and $\Phi(B)=$ $B_{1}, \ldots, B_{n}$ and $\Phi(C)=C_{1}, \ldots, C_{m}$, then by induction hypothesis we have $\left|s^{*}\right|_{(\mu, 1)} \sim \Phi(B)^{+} \sim\left(\left(\ldots\left(B_{1} \vee B_{2}\right) \ldots \vee B_{n}\right)\right.$ and $\left|s^{*}\right|_{(\mu, 2)} \sim$ $\Phi(C)^{-} \sim\left(\left(\ldots\left(C_{1} \wedge C_{2}\right) \ldots \wedge C_{n}\right)\right.$. Hence, by distributivity of $\rightarrow$ with respect to $\vee$ and $\wedge,\left|s^{*}\right|_{\mu} \sim\left(\left(\ldots\left(\left(\ldots\left(B_{1} \rightarrow C_{1}\right) \wedge \ldots\right) \wedge\left(B_{1} \rightarrow\right.\right.\right.\right.$ $\left.\left.C_{m}\right)\right) \ldots \wedge\left(\left(\ldots\left(B_{n} \rightarrow C_{1}\right) \wedge \ldots\right) \wedge\left(B_{n} \rightarrow C_{m}\right)\right)$. Therefore, $\left|s^{*}\right|_{\mu} \sim$ $\Phi(A)^{-}=B_{1} \rightarrow C_{1}, \ldots, B_{1} \rightarrow C_{m}, \ldots, B_{n} \rightarrow C_{1}, \ldots, B_{n} \rightarrow C_{m}$. 
Lemma 4.7 (Validity of the Extracted Sequent). Let $\varphi$ be an LK-Proof. Then $H_{L K_{A}}(\varphi)$ is valid.

Proof. By lemma 4.6, $H_{L K_{A}}(\varphi)$ and $\left|\Phi_{P}(\Psi(\varphi))\right|_{[\epsilon]}$ are logically equivalent, and $\left|\Phi_{P}(\Psi(\varphi))\right|_{[\epsilon]}$ is valid because algorithm 4.5 constructs a proof for it. Therefore, $H_{L K_{A}}(\varphi)$ is also valid.

Theorem 4.8 (Soundness of Algorithm 4.4). Let $\varphi$ be an LK-Proof. Then the sequent extracted by algorithm $4.4, H_{L K_{A}}(\varphi)$, is a Herbrand sequent.

Proof. Lemmas 4.5 and 4.7 show that the extracted sequent satisfies the requirements in definition 3.3.2 and thus it is a Herbrand sequent.

Definition 4.2.4 (Herbrand Sequent of an LK-Proof with End-sequent in Non-Prenex Form). The determinism of algorithm 4.4 and theorem 4.8 together allow us to define The Herbrand Sequent of an $\mathbf{L K}$-Proof $\varphi$ with End-Sequent in Non-Prenex Form, $H_{P}(\varphi)$, as the very result of the algorithm, $H_{L K_{A}}(\varphi)$.

Example 4.7 (Extraction of Herbrand Sequent via Transformation from $\mathbf{L K}$ to $\left.\mathbf{L} \mathbf{K}_{\mathbf{A}}\right)$. Let $\varphi$ be the $\mathbf{L K}$-Proof below. It is slightly modified with respect to the $\mathbf{L K}$-Proof $\varphi$ of example 4.2, because it has an end-sequent in non-prenex form, obtained from the end-sequent in example 4.2 by the additional rule $(\wedge: l)$.

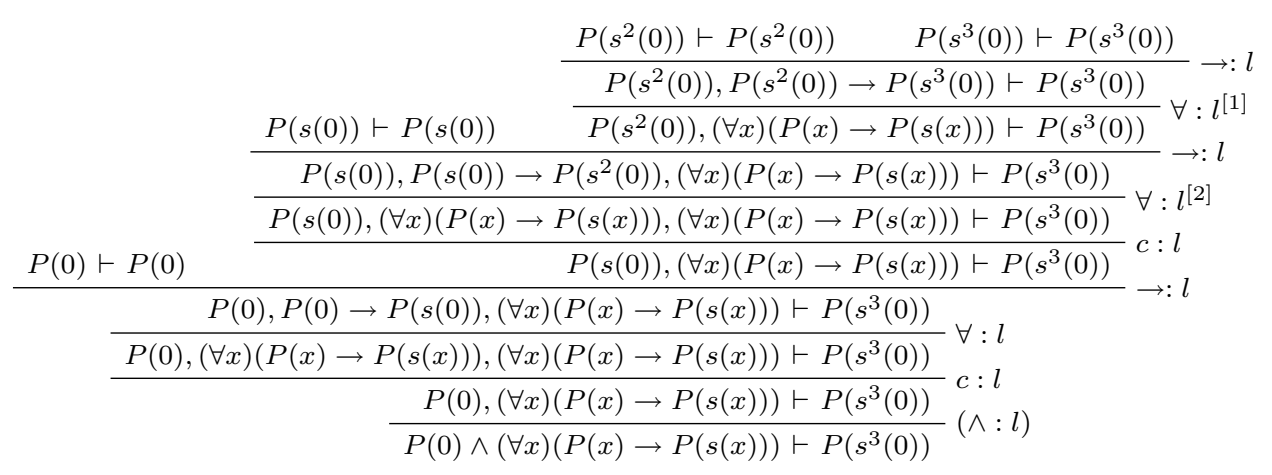

Since its end-sequent is not in prenex-form, Mid-Sequent Reduction cannot be used to extract its Herbrand sequent. As an alternative way to extract its Herbrand sequent, we can transform $\varphi$ into its $\mathbf{L K}_{\mathbf{A}}-\operatorname{Proof} \Psi(\varphi)$, as shown below.

First we have to omit quantifier rules from $\varphi$, thus obtaining the following LK-Proof with false contractions. 


$$
\frac{P(0) \vdash P(0) \quad \frac{P(s(0)) \vdash P(s(0)) \quad \frac{P\left(s^{2}(0)\right) \vdash P\left(s^{2}(0)\right)}{P\left(s^{2}(0)\right), P\left(s^{2}(0)\right) \rightarrow P\left(s^{3}(0)\right) \vdash P\left(s^{3}(0)\right)}}{\frac{P(s(0)), P(s(0)) \rightarrow P\left(s^{2}(0)\right), P\left(s^{2}(0)\right) \rightarrow P\left(s^{3}(0)\right) \vdash P\left(s^{3}(0)\right)}{P(s(0)),(\forall x)(P(x) \rightarrow P(s(x))) \vdash P\left(s^{3}(0)\right)}} c: l}{} \rightarrow: l
$$

Then we replace. starting from above and going downwards, the 2 false contractions, $(c: l)$-rules, by $(\langle\langle: l)$-rules. The resulting $\Psi(\varphi)$ is:

\begin{tabular}{|c|c|c|}
\hline & \multirow[b]{2}{*}{$P(s(0)) \vdash P(s(0))$} & $P\left(s^{2}(0)\right) \vdash P\left(s^{2}(0)\right)$ \\
\hline \multirow{2}{*}{$P(0) \vdash P(0)$} & & $P\left(s^{2}(0)\right), P\left(s^{2}(0)\right) \rightarrow P\left(s^{3}(0)\right) \vdash P\left(s^{3}(0)\right)$ \\
\hline & \multicolumn{2}{|c|}{$\frac{P(s(0)), P(s(0)) \rightarrow P\left(s^{2}(0)\right), P\left(s^{2}(0)\right) \rightarrow P\left(s^{3}(0)\right) \vdash P\left(s^{3}(0)\right)}{P(s(0)),\left\langle P(s(0)) \rightarrow P\left(s^{2}(0)\right), P\left(s^{2}(0)\right) \rightarrow P\left(s^{3}(0)\right)\right\rangle \vdash P\left(s^{3}(0)\right)}\langle\rangle: l \mid l$} \\
\hline \multicolumn{3}{|c|}{$P(0), P(0) \rightarrow P(s(0)),\left\langle P(s(0)) \rightarrow P\left(s^{2}(0)\right), P\left(s^{2}(0)\right) \rightarrow P\left(s^{3}(0)\right)\right\rangle \vdash P\left(s^{3}(0)\right)$} \\
\hline \multicolumn{3}{|c|}{$P(0),\left\langle P(0) \rightarrow P(s(0)),\left\langle P(s(0)) \rightarrow P\left(s^{2}(0)\right), P\left(s^{2}(0)\right) \rightarrow P\left(s^{3}(0)\right)\right\rangle\right\rangle \vdash P\left(s^{3}(0)\right)$} \\
\hline
\end{tabular}

We can then extract the Herbrand sequent of $\Psi(\varphi)$ by:

$$
\begin{aligned}
& H(\Psi(\varphi))=\Phi\left(|\Psi(\varphi)|_{[\epsilon]}\right) \\
& =\Phi\left(P(0) \wedge\left\langle\left\langle\begin{array}{c}
P(0) \rightarrow P(s(0)), \\
P(s(0)) \rightarrow P\left(s^{2}(0)\right), \\
P\left(s^{2}(0)\right) \rightarrow P\left(s^{3}(0)\right)
\end{array}\right\rangle \vdash P\left(s^{3}(0)\right)\right)\right. \\
& \left.=\Phi\left(P(0) \wedge\left\langle\begin{array}{c}
P(0) \rightarrow P(s(0)), \\
P(s(0)) \rightarrow P\left(s^{2}(0)\right), \\
P\left(s^{2}(0)\right) \rightarrow P\left(s^{3}(0)\right)
\end{array}\right\rangle\right)\right) \vdash P\left(s^{3}(0)\right) \\
& =\left(\Phi\left(P(0) \wedge\left\langle\begin{array}{c}
P(0) \wedge P(0) \rightarrow P(s(0)), \\
P(s(0)) \rightarrow P\left(s^{2}(0)\right), \\
P\left(s^{2}(0)\right) \rightarrow P\left(s^{3}(0)\right)
\end{array}\right\rangle\right) \mapsto P\left(s^{3}(0)\right)\right. \\
& =\left(\begin{array}{c}
P(0) \wedge P(0) \rightarrow P(s(0)), \\
P(0) \wedge P(s(0)) \rightarrow P\left(s^{2}(0)\right), \\
P(0) \wedge P\left(s^{2}(0)\right) \rightarrow P\left(s^{3}(0)\right)
\end{array}\right) \vdash P\left(s^{3}(0)\right)
\end{aligned}
$$




\subsubsection{Extraction via Collection of Sub-Formula Instances}

The algorithm described in the previous subsection is able to extract a Herbrand sequent for a non-prenex proof, but it has the same disadvantage that the mid-sequent reduction algorithm had for the prenex case: it needs to transform the whole proof. In this subsection we describe an algorithm that extracts a Herbrand sequent for the non-prenex case without transforming the proof. This algorithm, which we call Herbrand Sequent Extraction by Sub-Formula Instance Collection, generalizes algorithm 4.2 using some ideas of algorithm 4.4.

We notice that algorithm 4.2 collects all the instances of all quantified occurrences of the end-sequent and then it constructs a Herbrand sequent by removing all those quantified occurrences of the end-sequent and inserting all the collected instances. However, in the non-prenex case, one difficulty arises: we need to substitute collected instances not for occurrences in the end-sequent, but for specific sub-formulas in the end-sequent. This requires two modifications:

1. we need to keep track of which instances belong to which sub-formula and collect them separately. We do this by introducing labels in the LK-Proof and the notion of rule-reference to a sub-formula.

2. since a quantified sub-formula in position $\lambda$ may have more than one instance, as a result of possible contractions on its ancestors, we may need to substitute all these instances in the same place, in the same position $\lambda$. We solve this by grouping all these instances in a temporary array-formula. Later we expand the whole formula that contains the array-formula into a sequence of standard First-Order-Logic formulas, using the already defined function $\Phi$ to eliminate the temporary arrays.

Although the essence of the generalization, as explained above, is relatively simple, the formal specification of the algorithm requires many extensions of the definitions of positions and substitutions to deal with the temporary array-formulas. Among these extensions, the most important is the concept of unifying substitution. When dealing with LK-Proofs containing quantifiers nested in the scope of other quantifiers, it may happen that substituting formulas do not unify with the sub-formulas they were supposed to substitute, and these substitutions should be avoided, because performing them would imply the deletion or corruption of instantiations due to another contracted copy of the outer quantified formula or to another branch of the tree. Unifying substitutions must distinguish different contracted copies of a sub-formula, and in order for this to be possible, we also define markers for sub-formulas. Example 4.17 shows an LK-Proof with nested quantifiers in which unifying substitutions play a big role.

As for the previous algorithm, Extraction via Transformation to $\mathbf{L K}_{\mathbf{A}}$, in this algorithm it is also assumed that the proofs are skolemized. 
Definition 4.2.5 (Position-labeled Sequent). A position-labeled sequent $s$ is a sequent in which each occurrence is labeled with a position $(\mu, \lambda)$, where $\mu$ is a position of a occurrence in $s$ and $\lambda$ is a position of a sub-formula in this occurrence.

Definition 4.2.6 (Position-labeled LK-Proof). A position-labeled LK-Proof is defined recursively as an LK-Proof in which:

- Each occurrence in position $\mu_{i}$ in the end-sequent is labeled with $\left(\mu_{i}, \varepsilon\right)$.

- For each rule, the context occurrences in the premise have the same labels as their corresponding occurrences in the conclusion.

- For each rule, let $\left(\mu_{m}, \lambda_{m}\right)$ be the label of its main occurrence. Then its auxiliary occurrences are labeled with $\left(\mu_{m}, \lambda_{m} \cdot j\right)$, where $j$ is the position of main occurrence's sub-formula corresponding to the auxiliary occurrence.

- Cut-formulas (and their ancestors) are not labeled.

Example 4.8 (Position-labeled LK-Proof). The LK-Proof $\varphi$ below is shown with position labels in the upper-right corner of each occurrence:

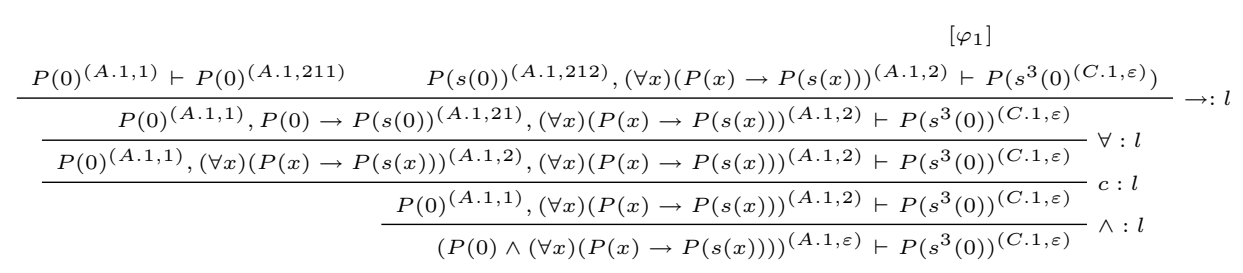

where $\varphi_{1}$ is:

$$
\frac{\frac{P(s(0))^{(A .1,212)} \vdash P(s(0))^{(A .1,211)}}{\frac{\left[\varphi_{2}\right]}{P(s(0))^{(A .1,212)}, P(s(0)) \rightarrow P\left(s^{2}(0)\right)^{(A .1,21)},(\forall x)(P(x) \rightarrow P(s(x)))^{(A .1,2)} \vdash P\left(s^{3}(0)\right)^{(C .1, \varepsilon)}}}}{\frac{P(s(0))^{(A .1,212)},(\forall x)(P(x) \rightarrow P(s(x)))^{(A .1,2)},(\forall x)(P(x) \rightarrow P(s(x)))^{(A .1,2)} \vdash P\left(s^{3}(0)\right)^{(C .1, \varepsilon)}}{P(s(0))^{(A .1,212)},(\forall x)(P(x) \rightarrow P(s(x)))^{(A .1,2)} \vdash P\left(s^{3}(0)^{(C .1, \varepsilon)}\right)}} \quad \forall: l l^{[2]}
$$

where $\varphi_{2}$ is:

$$
\frac{P\left(s^{2}(0)\right)^{(A .1,212)} \vdash P\left(s^{2}(0)\right)^{(A .1,211)} \quad P\left(s^{3}(0)\right)^{(A .1,212)} \vdash P\left(s^{3}(0)\right)^{(C .1, \varepsilon)}}{\frac{P\left(s^{2}(0)\right)^{(A .1,212)}, P\left(s^{2}(0)\right) \rightarrow P\left(s^{3}(0)\right)^{(A .1,21)} \vdash P\left(s^{3}(0)\right)^{(C .1, \varepsilon)}}{P\left(s^{2}(0)\right)^{(A .1,212)},(\forall x)(P(x) \rightarrow P(s(x)))^{(A .1,2)} \vdash P\left(s^{3}(0)\right)^{(C .1, \varepsilon)}} \forall: l l} \quad \forall: l
$$

Definition 4.2.7 (Marking Atomic Sub-Formulas: Contractions and Depth). The marker of an atomic sub-formula $F$ occurring in position $[\nu, \mu, \lambda]$ of a labeled LK-Proof $\varphi$ is denoted by $\mathcal{M}([\nu, \mu, \lambda])$, and it is a string belonging to $\mathbb{N}^{*}$. All sub-formulas from $\varphi$ are marked, starting from the bottom and going upwards, according to the inductive rules below:

- Base Case 1: If $F$ is an atomic sub-formula in the end-sequent $(\nu=\varepsilon)$, then $\mathcal{M}([\nu, \mu, \lambda])=1$. 
- Base Case 2: If $F$ is an atomic cut-formula, then $\mathcal{M}([\nu, \mu, \lambda])=1$.

- Inductive Case: Let $\rho$ be a rule in $\varphi$ and let $\bar{m}$ be the marker of a sub-formula $F$ in its conclusion sequent. If $\rho$ is a contraction rule and $F$ is its main occurrence, then we mark all ancestors $F_{j}$ of $F$ with $\bar{m} j$. Otherwise, we mark the ancestors of $F$ with $\bar{m} 1$.

Example 4.9 (Marking Atomic Sub-Formulas). In the proof $\varphi$ below, we show the marker of each atomic sub-formula as strings subscripted to the predicate symbol.

$$
\begin{aligned}
& \frac{P_{1111111}(a, b) \vdash P_{1111111}(a, b) \quad P_{1211111}(a, c) \vdash P_{1111111}(a, c)}{\frac{P_{111111}(a, b), P_{121111}(a, c) \vdash P_{111111}(a, b) \wedge P_{111111}(a, c)}{P_{11111}(a, b),(\forall y)\left(P_{12111}(a, y)\right) \vdash P_{11111}(a, b) \wedge P_{11111}(a, c)} \forall: ~} \wedge: l
\end{aligned}
$$

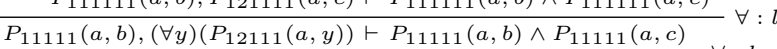

$$
\begin{aligned}
& \overline{(\forall y)\left(P_{1111}(a, y)\right),(\forall y)\left(P_{1211}(a, y)\right) \vdash P_{1111}(a, b) \wedge P_{1111}(a, c)} \forall: l \\
& \frac{(\forall y)\left(P_{111}(x, y)\right),(\forall x)(\forall y)\left(P_{121}(x, y)\right) \vdash P_{111}(a, b) \wedge P_{111}(a, c)}{\forall} \forall: l \\
& \frac{\overline{(\forall x)(\forall y)\left(P_{11}(x, y)\right),(\forall x)(\forall y)\left(P_{12}(x, y)\right) \vdash P_{11}(a, b) \wedge P_{11}(a, c)}}{(\forall x)(\forall y)\left(P_{1}(x, y)\right) \vdash P_{1}(a, b) \wedge P_{1}(a, c)} c: l
\end{aligned}
$$

The markers provide us with two kinds of information:

- The length of the marker informs the depth in the proof-tree in which the sub-formula occurs.

- Whenever a contraction occurs in a given depth of the tree, we have numbers different from 1 in the corresponding position of the marker. These numbers keep track of different copies of the atomic sub-formula that were contracted.

Definition 4.2.8 (Marking Atomic Sub-Formulas: Contractions and Contraction-Depth). The contraction-marker of an atomic sub-formula $F$ occurring in position $[\nu, \mu, \lambda]$ of a labeled LK-Proof $\varphi$ is denoted by $\mathcal{M}_{\mathcal{C}}([\nu, \mu, \lambda])$, and it is a string belonging to $\mathbb{N}^{*}$. All sub-formulas from $\varphi$ are marked, starting from the bottom and going upwards, according to the inductive rules below:

- Base Case 1: If $F$ is an atomic sub-formula in the end-sequent $(\nu=\varepsilon)$, then $\mathcal{M}_{\mathcal{C}}([\nu, \mu, \lambda])=\varepsilon$.

- Base Case 2: If $F$ is an atomic cut-formula, then $\mathcal{M}_{\mathcal{C}}([\nu, \mu, \lambda])=\varepsilon$.

- Inductive Case: Let $\rho$ be a rule in $\varphi$ and let $\bar{m}$ be the marker of a sub-formula $F$ in its conclusion sequent. If $\rho$ is a contraction rule and $F$ is its main occurrence, then we mark all ancestors $F_{j}$ of $F$ with $\bar{m} j$. Otherwise, we mark the ancestors of $F$ with $\bar{m}$.

The length of the contraction-marker of a sub-formula is called contractiondepth of the sub-formula. 
Example 4.10 (Marking Atomic Sub-Formulas: Contractions and Contraction-Depth). Considering example 4.9, we notice that the markers serve the purpose of distinguishing contracted copies, but since we are usually not interested in the depth of sub-formulas, those sequences of 1 generated whenever a sub-formula in the conclusion has only one copy in the premises is useless and only prevents the easy reading of the interesting information contained in the markers. Marking with Contraction-markers, however, adds new numbers to the string only when a sub-formula has more than one copy in the premises, making it easier to read and distinguish contracted copies. The LK-Proof below is the same shown in example 4.9, but with contractionmarkers instead of simple markers:

$$
\begin{gathered}
\frac{P_{1}(a, b) \vdash P(a, b) \quad P_{2}(a, c) \vdash P(a, c)}{P_{1}(a, b), P_{2}(a, c) \vdash P(a, b) \wedge P(a, c)} \wedge: l \\
\frac{\frac{P_{1}(a, b),(\forall y)\left(P_{2}(a, y)\right) \vdash P(a, b) \wedge P(a, c)}{(\forall y)\left(P_{1}(a, y)\right),(\forall y)\left(P_{2}(a, y)\right) \vdash P(a, b) \wedge P(a, c)}}{\frac{(\forall y)\left(P_{1}(x, y)\right),(\forall x)(\forall y)\left(P_{2}(x, y)\right) \vdash P(a, b) \wedge P(a, c)}{(\forall x)(\forall y)\left(P_{1}(x, y)\right),(\forall x)(\forall y)\left(P_{2}(x, y)\right) \vdash P(a, b) \wedge P(a, c)}} \forall: l \\
\left.\frac{(\forall x)(\forall y)(P(x, y)) \vdash P(a, b) \wedge P(a, c)}{(\forall}\right) \\
\forall: l
\end{gathered}
$$

Contraction-markers provide us with two kinds of information:

- The length of the marker, the contraction-depth, informs the number of contractions (implicit or explicit) suffered by the sub-formula in the path from the sub-formula to its terminal formula (a formula in the end-sequent or a cut-formula).

- Whenever a contraction occurs the copies being contracted have different numbers in the last position of their contraction-markers. This allow us to distinguish them.

Definition 4.2.9 (Rule-Reference to Sub-formula). We say that a rule refers to a sub-formula in position $\lambda$ of a formula occurrence in position $\mu$ of the end-sequent if and only if its main occurrence is labeled with $(\mu, \lambda)$.

Definition 4.2.10 (Set of Rules Referring to a Sub-Formula). $Q_{(\mu, \lambda)}(\varphi)$ is the set of all rules of the LK-Proof $\varphi$ referring to the sub-formula in position $\lambda$ of the formula occurrence in position $\mu$ in the end-sequent.

Definition 4.2.11 (Sets of Used Auxiliary Occurrences). Let $R$ be a set of rules. With $\mathcal{A}(R)$ we denote the set of used auxiliary occurrences in the rules in $\mathrm{R}$.

Definition 4.2.12 (Set to Array Formation). Let $M$ be a set of occurrences. With $\mathcal{S}(M)$ we denote the array-formula formed with the occurrences in $M$.

Definition 4.2.13 (Array-Formula Referring to a Sub-Formula). Let $\lambda$ be a position in an occurrence in position $\mu$ of the end-sequent of a proof $\varphi$. 
Then we define the array-formula referring to the sub-formula in position $\lambda$ of the occurrence indexed with $\mu$ in the end-sequent:

$$
\left.Q_{(\mu, \lambda)}^{A}(\varphi) \doteq \mathcal{S}\left(\mathcal{A}\left(Q_{(\mu, \lambda)}(\varphi)\right)\right)\right)
$$

Definition 4.2.14 (Dequantification of a Sequent). Let $s$ be a sequent with all variables standardized apart. Then $\mathcal{D}(s)$ denotes the sequent obtained from $s$ by removing all quantifiers and substituting all the variables that were bound by these quantifiers by distinct eigen-constants.

Definition 4.2.15 (Positions of an Array-Formula). Let $A$ be an arrayformula, then its set of positions pos $(A)$ is the smallest set of strings belonging to $\{1,2\}^{*}$ and such that $\operatorname{pos}(A)$ satisfies:

1. $\varepsilon \in \operatorname{pos}(A)$

2. If $A$ is of the form $\neg B$, then $\{1 . \lambda \mid \lambda \in \operatorname{pos}(B)\} \subset \operatorname{pos}(A)$.

3. If $A$ is of the form $(B \wedge C)$, then $\left(\left\{1 . \lambda_{1} \mid \lambda_{1} \in \operatorname{pos}(B)\right\} \cup\left\{2 . \lambda_{2} \mid \lambda_{2} \in\right.\right.$ $\operatorname{pos}(C)\}) \subset \operatorname{pos}(A)$.

4. If $A$ is of the form $(B \vee C)$, then $\left(\left\{1 . \lambda_{1} \mid \lambda_{1} \in \operatorname{pos}(B)\right\} \cup\left\{2 . \lambda_{2} \mid \lambda_{2} \in\right.\right.$ $\operatorname{pos}(C)\}) \subset \operatorname{pos}(A)$.

5. If $A$ is of the form $(B \rightarrow C)$, then $\left(\left\{1 . \lambda_{1} \mid \lambda_{1} \in \operatorname{pos}(B)\right\} \cup\left\{2 . \lambda_{2} \mid \lambda_{2} \in\right.\right.$ $\operatorname{pos}(C)\}) \subset \operatorname{pos}(A)$.

6. If $A$ is of the form $(\forall x) B$, then $\{1 . \lambda \mid \lambda \in \operatorname{pos}(B)\} \subset \operatorname{pos}(A)$.

7. If $A$ is of the form $(\exists x) B$, then $\{1 . \lambda \mid \lambda \in \operatorname{pos}(B)\} \subset \operatorname{pos}(A)$.

8. If $A$ is of the form $\left\langle A_{1}, A_{2}, \ldots, A_{n}\right\rangle$, then $\left\{1 . \lambda \mid \lambda \in \operatorname{pos}\left(A_{j}\right)\right.$ for some $j \in$ $\{1, \ldots, n\}\} \subset \operatorname{pos}(A)$

Definition 4.2.16 (Multi-sets of Sub-formulas occurring in given Positions of an Array-Formula). Let $\lambda$ be a position of the array-formula $A$. Then the multi-set of sub-formulas occurring in position $\lambda$ is denoted $|A|_{\lambda}$. Formally, we may define the computation of $|A|_{\lambda}$ inductively:

1. $|A|_{\varepsilon} \doteq\{A\}$

2. If $A$ is of the form $\neg B$ and $\lambda=1$. $\lambda^{\prime}$ with $\lambda^{\prime} \in \operatorname{pos}(B)$, then $|A|_{\lambda} \doteq$ $|B|_{\lambda^{\prime}}$.

3. If $A$ is of the form $B \circ C$ (for $\circ \in\{\wedge, \vee, \rightarrow\}$ ) and $\lambda=1 . \lambda^{\prime}$ with $\lambda^{\prime} \in \operatorname{pos}(B)$, then $|A|_{\lambda} \doteq|B|_{\lambda^{\prime}}$.

4. If $A$ is of the form $B \circ C$ (for $\circ \in\{\wedge, \vee, \rightarrow\}$ ) and $\lambda=2 \cdot \lambda^{\prime}$ with $\lambda^{\prime} \in \operatorname{pos}(C)$, then $|A|_{\lambda} \doteq|C|_{\lambda^{\prime}}$. 
5. If $A$ is of the form $(Q x) B$ (for $Q \in\{\forall, \exists\}$ ) and $\lambda=1 . \lambda^{\prime}$ with $\lambda^{\prime} \in \operatorname{pos}(B)$, then $|A|_{\lambda} \doteq|B|_{\lambda^{\prime}}$.

6. If $A$ is of the form $\left\langle A_{1}, A_{2}, \ldots, A_{n}\right\rangle$, and $\lambda=1 . \lambda^{\prime}$, then $|A|_{\lambda} \doteq \bigcup_{\lambda^{\prime} \in \operatorname{pos}\left(A_{j}\right), j \in\{1, \ldots, n\}}\left\{\left|A_{j}\right|_{\lambda^{\prime}}\right\}$.

Example 4.11 (Multi-sets of Sub-Formulas occurring in given Positions of an Array-Formula). Let $A \doteq\langle P(a) \wedge(\forall y)(Q(a, y) \rightarrow Q(a, s(y))), P(b) \wedge$ $(\forall y)(Q(b, y) \rightarrow Q(b, s(y))))\rangle$ be an array-formula. Then:

$$
\begin{gathered}
\mid A_{\varepsilon}=\{\langle P(a) \wedge(\forall y)(Q(a, y) \rightarrow Q(a, s(y))), P(b) \wedge(\forall y)(Q(b, y) \rightarrow Q(b, s(y)))\rangle\} \\
|A|_{11}=\{P(a), P(b)\} \\
|A|_{12}=\{(\forall y)(Q(a, y) \rightarrow Q(a, s(y))),(\forall y)(Q(b, y) \rightarrow Q(b, s(y)))\}
\end{gathered}
$$

Definition 4.2.17 (Sub-Formula Substitutions in Array-Formulas). Let $\lambda$ be a position of the array-formula $A$ such that $|A|_{\lambda}=\left\{B_{1}, B_{2}, \ldots, B_{n}\right\}$. The array-formula obtained by substituting the $B_{j}$ in position $\lambda$ in $A$ by $C$ is denoted $A_{\lambda}[C]$ or $A \sigma^{f o}$, where $\sigma^{f o} \doteq\{\lambda / C\}$.

Example 4.12 (Sub-Formula Substitutions in Array-Formulas). Let $A=$ $\langle P(a) \wedge(\forall y)(Q(a, y)), P(b) \wedge(\forall y)(Q(b, y))\rangle$ be an array-formula. Then:

$$
A\{2 / Q(a, 0)\}=\langle P(a) \wedge Q(a, 0), P(b) \wedge Q(a, 0)\rangle
$$

Definition 4.2.18 (Marker-Unification). Two formulas $A$ and $B$ markerunify, Unifiable ${ }_{M}(A, B)$, if and only if they unify and the contractionmarkers of the atomic sub-formulas of $A$ (or $B$ ) are prefix substrings of the contraction-markers of the corresponding sub-formulas of $B$ (or $A$ ). Markerunification is thus unification aware of contraction-markers.

Example 4.13 (Marker-unification). Marker-unification is standard unification extended to formulas with contraction-markers, as exemplified below:

- Unifiable $_{M}(P(a), P(x))$.

- Unifiable ${ }_{M}\left(P_{12}(a), P(x)\right)$.

- Unifiable ${ }_{M}\left(P_{12}(a), P_{1}(x)\right)$.

- $\neg$ Unifiable $_{M}\left(P_{12}(a), P_{2}(x)\right)$.

- Unifiable ${ }_{M}\left(Q_{12}(a) \wedge P_{12}(a), Q_{1}(a) \wedge P_{1}(x)\right)$.

- $\neg$ Unifiable $_{M}\left(Q_{12}(a) \wedge P_{12}(a), Q_{2}(a) \wedge P_{2}(x)\right)$. 
Definition 4.2.19 (Unifying Substitutions in Array-Formulas). Let $\lambda$ be a position of the array-formula $A$ such that $|A|_{\lambda}=\left\{B_{1}, B_{2}, \ldots, B_{n}\right\}$. Let $C \doteq$ $\left\langle C_{1}, \ldots, C_{m}\right\rangle$. Let $A_{Q} \doteq\left\{j \mid B_{j}\right.$ ( is of the form $\left.(Q x) B_{j}^{\prime}\right\}$ with $Q \in\{\forall, \exists\}$. For $j \in A_{Q}$, let $U_{j} \doteq\left\{\left.C_{k}\left|C_{k} \in\right| C\right|_{1}\right.$ and Unifiable $\left.{ }_{M}\left(C_{k}, B_{j}^{\prime}\right)\right\}$. Let $A_{Q}^{\prime} \doteq$ $\left\{j \mid U_{j} \neq \emptyset\right\}$. then, by $\overline{A \sigma^{f o}}$, where $\overline{\sigma^{f o}} \doteq \overline{\{\lambda / C\}}$, we denote the arrayformula obtained by substituting in $A$ those $B_{j}\left(j \in A_{Q}^{\prime}\right)$ in position $\lambda$ by the array formula $\mathcal{S}\left(U_{j}\right)$.

Furthermore, $\overline{\sigma^{f s}}$ and $\overline{\sigma^{f p}}$ are defined analogously.

Example 4.14 (Unifying Substitutions in Array-Formulas). Let $A=\langle P(a) \wedge$ $(\forall y)(Q(a, y)), P(b) \wedge(\forall y)(Q(b, y))\rangle$ be an array-formula. Then:

$$
A \overline{\{2 / Q(a, 0)\}}=\langle P(a) \wedge Q(a, 0), P(b) \wedge(\forall y)(Q(b, y))\rangle
$$

because $Q(a, 0)$ unifies with $Q(a, y)$, but not with $Q(b, y)$. The difference between usual substitutions and unifying substitutions can be seen by comparing this example with example 4.12

Example 4.15 (Unifying Substitutions with Contraction-markers). Let $A \doteq$ $(\forall x)(\forall y) P(x, y)$. Then:

$$
\begin{gathered}
A \overline{\left\{\varepsilon /\left\langle(\forall y) P_{1}(a, y),(\forall y) P_{2}(a, y)\right\rangle\right\}}=\left\langle(\forall y) P_{1}(a, y),(\forall y) P_{2}(a, y)\right\rangle \\
A \overline{\left\{\varepsilon /\left\langle(\forall y) P_{1}(a, y),(\forall y) P_{2}(a, y)\right\rangle\right\} \circ\left\{1 /\left\langle P_{1}(a, b), P_{2}(a, c)\right\rangle\right\}}=\left\langle\left\langle P_{1}(a, b)\right\rangle,\left\langle P_{2}(a, c)\right\rangle\right\rangle \\
A \overline{\left\{\varepsilon /\left\langle(\forall y) P_{1}(a, y),(\forall y) P_{2}(a, y)\right\rangle\right\} \circ\left\{1 /\left\langle P_{1}(a, b)\right\rangle\right\} \circ\left\{1 /\left\langle P_{2}(a, c)\right\rangle\right\}}=\left\langle\left\langle P_{1}(a, b)\right\rangle,\left\langle P_{2}(a, c)\right\rangle\right\rangle
\end{gathered}
$$

For the sake of contrast, without contraction-markers we would have:

$$
\begin{gathered}
A \overline{\{\varepsilon /\langle(\forall y) P(a, y),(\forall y) P(a, y)\rangle\}}=\langle(\forall y) P(a, y),(\forall y) P(a, y)\rangle \\
A \overline{\{\varepsilon /\langle(\forall y) P(a, y),(\forall y) P(a, y)\rangle\} \circ\{1 /\langle P(a, b), P(a, c)\rangle\}}=\langle\langle P(a, b), P(a, c)\rangle,\langle P(a, b), P(a, c)\rangle\rangle \\
A \overline{\{\varepsilon /\langle(\forall y) P(a, y),(\forall y) P(a, y)\rangle\} \circ\{1 /\langle P(a, b)\rangle\} \circ\{1 /\langle P(a, c)\rangle\}}=\langle\langle P(a, b)\rangle,\langle P(a, b)\rangle\rangle
\end{gathered}
$$

By comparing the last substitutions of the cases with and without contraction-markers, we can see their importance. Without contraction-markers, the instantiation of $(\forall y) P(a, y)$ to $P(a, c)$ was lost, because $P(a, y)$ was firstly unified with $P(a, b)$ substituted by it.

Algorithm 4.6 (Extraction of Herbrand Sequent without Proof-Transformation for Proofs with Non-prenex End-sequents). Let $\varphi$ be a Positionlabeled LK-Proof, and let $s$ be its end-sequent.

Let:

$$
\begin{gathered}
S_{Q S F}(s) \doteq\left\{\left(\mu_{i}, \lambda_{i}\right) \mid \lambda_{i} \text { is a position such that }|s|_{\left(\mu_{i}, \lambda_{i}\right)}\right. \text { is a } \\
\text { sub-formula starting with a quantifier }\}
\end{gathered}
$$


For each position $\left(\mu_{i}, \lambda_{i}\right) \in S_{Q S F}(s)$, let its corresponding substitution be:

$$
\sigma_{\left(\mu_{i}, \lambda_{i}\right)}^{f s} \doteq\left\{\left(\mu_{i}, \lambda_{i}\right) / Q_{\left(\mu_{i}, \lambda_{i}\right)}^{A}(\varphi)\right\}
$$

Then we compute the following sequent from $\varphi$ :

$$
H_{A}(\varphi) \doteq \operatorname{set}\left(P\left(\Phi\left(\overline{\left(\sigma_{\left(\mu_{0}, \lambda_{0}\right)}^{f s} \circ \ldots \circ \sigma_{\left(\mu_{n}, \lambda_{n}\right)}^{f s}\right)}\right)\right)\right)
$$

where: $\operatorname{depth}\left(\lambda_{i}\right)<\operatorname{depth}\left(\lambda_{j}\right)$, if $i<j$ and $\mu_{i}=\mu_{j}{ }^{1}$

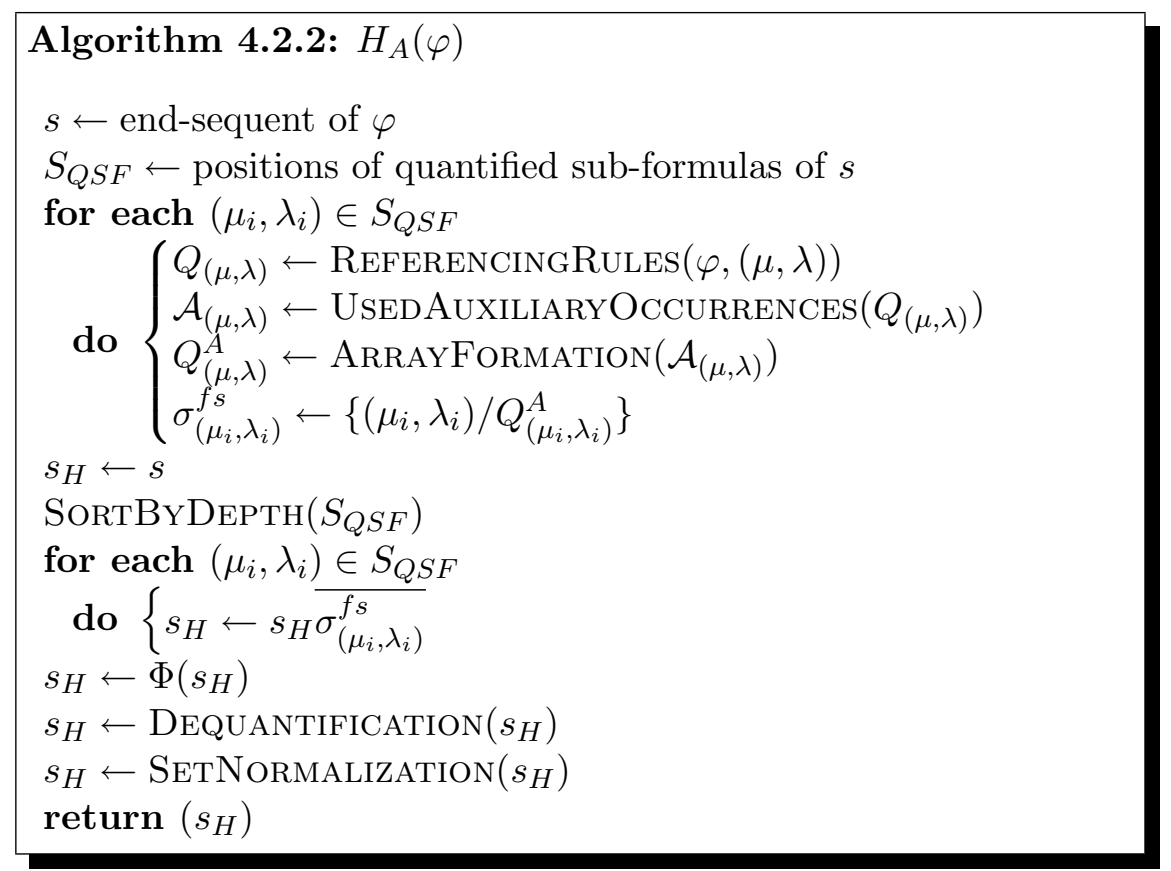

Lemma 4.8 (Extracted Sequent Well-Instantiated). Let $\varphi$ be an LK-Proof and let its end-sequent be $|\varphi|_{[\epsilon]}=A_{1}, \ldots, A_{n} \vdash B_{1}, \ldots, B_{m}$. $A_{1}^{0}, \ldots, A_{n}^{0}, B_{1}^{0}, \ldots, B_{m}^{0}$ are its formulas without quantifiers. Then the sequent extracted by algorithm 4.6 has the form:

$$
H_{A}(\varphi)=A_{1}^{1}, \ldots, A_{1}^{i_{1}}, \ldots A_{n}^{1}, \ldots, A_{n}^{i_{n}} \vdash B_{1}^{1}, \ldots, B_{1}^{p_{1}}, \ldots, B_{m}^{1}, \ldots, B_{m}^{p_{m}}
$$

where: $A_{j}^{1}, \ldots, A_{j}^{i_{j}}$ are quantifier-free substitution instances of $A_{j}^{0}$ and $B_{k}^{1}, \ldots, B_{k}^{p_{k}}$ are quantifier-free substitution instances of $B_{k}^{0}$.

Proof. By definition of $\Phi([1])$.

\footnotetext{
${ }^{1}$ This condition on the order of the composition of substitutions guarantees that variables bound by outer quantifiers are substituted before variables bound to nested inner quantifiers. This is important, because otherwise the substitution of an outer bounded variable would erase the substitution of an inner bounded variable.
} 
Lemma 4.9 (Validity of the Extracted Sequent). Let $\varphi$ be an LK-Proof. Then $H_{A}(\varphi)$ is valid.

Proof. Here we informally sketch a proof by induction.

In order to prove that $H_{A}(\varphi)$ is valid, we show a proof transformation that can be used to transform $\varphi$ into an LK-Proof $\varphi^{*}$ of $H_{A}(\varphi)^{2}$. This transformation progressively eliminates quantifier-rules from $\varphi$ and adjusts the sequents so that the resulting proof always remains a valid LK-Proof.

- Base Case; Quantifiers with depth 0:

For a quantifier with depth 0 in an occurrence in position $\mu$ of the endsequent, we transform the proof by deleting all quantifier-rules and all contraction-rules referring to $(\mu, \varepsilon)$ and by performing a unifying substitution of the sub-formula in position $(\mu, \varepsilon)$ by

$$
\Phi\left(Q_{(\mu, \varepsilon)}^{A}(\varphi)\right)
$$

- Induction Case; Quantifiers with depth $n+1$ :

Let $\varphi^{\prime}$ be the proof after all quantifiers up to depth $n$ have been eliminated. And we are now interested in eliminating a quantifier with depth $n+1$ in a sub-formula in position $(\mu, \lambda)$ in the end-sequent.

Let $\nu_{1}, \ldots, \nu_{n}$ be positions in $\varphi^{\prime}$ such that $\varphi_{\left[\nu_{i}\right]}^{\prime}$ has an ancestor occurrence of $\left|\varphi^{\prime}\right|_{[\varepsilon, \mu]}$ such that $\left|\varphi^{\prime}\right|_{\left[\nu, \mu_{i}, \varepsilon\right]}=\left|\varphi^{\prime}\right|_{[\varepsilon, \mu, \lambda]}$.

We transform $\left|\varphi^{\prime}\right|_{\nu_{i}}$ by deleting all quantifier-rules and contractionrules referring to $\left(\mu_{i}, \varepsilon\right)$ and by performing a unifying substitution ${ }^{3}$ of the sub-formula in position $\left(\mu_{i}, \varepsilon\right)$ by

$$
\Phi\left(Q_{(\mu, \lambda)}^{A}(\varphi)\right)
$$

Additionally, if the formula occurrence at position $\left[\nu_{i}, \mu_{i}, \epsilon\right]$ is an ancestor of some sub-formula $F_{s}$ in position $\lambda_{s}$ of an occurrence $F$, then we perform a unifying substitution of $F$ by

$$
\Phi\left(F \sigma_{s}^{f o}\right)
$$

where

$$
\sigma_{s}^{f o} \doteq\left\{\lambda_{s} / Q_{(\mu, \lambda)}^{A}(\varphi)\right\}
$$

\footnotetext{
${ }^{2}$ Alternatively, a similar, easier but more indirect, proof could be done by transforming $\varphi$ into a valid $\mathbf{L K}_{\mathrm{A}}$-Proof.

${ }^{3}$ We do a unifying substitution instead of just a normal substitution, because in general the sub-formula in position $\left(\mu_{i}, 1\right)$ may have had some of its variables instantiated by outer quantifiers and therefore it may not be unifiable with the formulas in $Q_{(\mu, \lambda)}^{A}(\varphi)$. In the case they do not unify, we do not want to perform the substitution, since this would affect the instantiation that was done by outer quantifiers.
} 
Let $\varphi_{1}$ be the result of applying the transformations above for all quantifiers in the end-sequent of $\varphi$. If some of the quantified sub-formulas of $\varphi$ were not used (in other words, if they don't have an ancestor in an axiom), then $\varphi_{1}$ will still contain some quantifiers in the end-sequent. We therefore transform $\varphi_{1}$ into $\varphi_{2}$ by dequantification (in other words, we substitute the remaining quantified variables in sub-formulas of the end-sequent and in their ancestor sub-formulas by eigen-constants).

Since $Q_{(\mu, \lambda)}^{A}(\varphi)$ is an array-formula which may have more than one formula possibly coming from different proof branches, $\varphi_{2}$ may have excessive formulas in some branches. The instantiation brings to one branch instantiated formulas that are actually necessary only in another branch. These excessive formulas must be generated and removed by inserting extra weakenings and by doubling some propositional rules in $\varphi_{2}$, in order to produce a well-formed LK-Proof $\varphi^{*}$.

By the way we constructed $\varphi^{*}$, eliminating the quantifiers from $\varphi$ by substituting them by the corresponding array formula with their instances, it is clear that the end-sequent of $\varphi^{*}$ is $H_{A}(\varphi)$.

Example 4.18 shows this process of transforming an LK-Proof $\varphi$ into an LK-Proof $\varphi^{*}$ for its extracted Herbrand sequent.

Example 4.16 (Extraction of Herbrand Sequent without Proof Transformation). Here we consider the same proof $\varphi$ of example 4.7, but now with labels, which however are shown below only for the end-sequent s:

$$
\begin{aligned}
& \begin{array}{ll}
\frac{P\left(s^{2}(0)\right) \vdash P\left(s^{2}(0)\right)}{P\left(s^{2}(0)\right), P\left(s^{2}(0)\right) \rightarrow P\left(s^{3}(0)\right) \vdash P\left(s^{3}(0)\right)} \\
\quad P(s(0)) \vdash P(s(0))
\end{array} \quad: l \\
& \frac{\frac{\frac{P(s(0)), P(s(0)) \rightarrow P\left(s^{2}(0)\right),(\forall x)(P(x) \rightarrow P(s(x))) \vdash P\left(s^{3}(0)\right)}{P(0) \vdash P(0)}}{\frac{P(s(0)),(\forall x)(P(x) \rightarrow P(s(x))),(\forall x)(P(x) \rightarrow P(s(x))) \vdash P\left(s^{3}(0)\right)}{P(s(0)),(\forall x)(P(x) \rightarrow P(s(x))) \vdash P\left(s^{3}(0)\right)}} \rightarrow: l}{l^{[2]}} \rightarrow: l
\end{aligned}
$$

Firstly we compute the set of positions corresponding to sub-formulas beginning with quantifiers in the quantifiers:

$$
S_{Q S F}(\varphi)=\{(A .1,2)\}
$$

Then we compute the sets of quantifier rules referring to the sub-formulas beginning with quantifiers. In our case, there is only one such a set, namely $Q_{(A .1,2)}(\varphi)$, with the following rules:

$$
\frac{P\left(s^{2}(0)\right), P\left(s^{2}(0)\right) \rightarrow P\left(s^{3}(0)\right) \vdash P\left(s^{3}(0)\right)}{P\left(s^{2}(0)\right),(\forall x)(P(x) \rightarrow P(s(x))) \vdash P\left(s^{3}(0)\right)} \forall: l^{[1]}
$$




$$
\begin{aligned}
& \frac{P(s(0)), P(s(0)) \rightarrow P\left(s^{2}(0)\right),(\forall x)(P(x) \rightarrow P(s(x))) \vdash P\left(s^{3}(0)\right)}{P(s(0)),(\forall x)(P(x) \rightarrow P(s(x))),(\forall x)(P(x) \rightarrow P(s(x))) \vdash P\left(s^{3}(0)\right)} \forall: l^{[2]} \\
& \frac{P(0), P(0) \rightarrow P(s(0)),(\forall x)(P(x) \rightarrow P(s(x))) \vdash P\left(s^{3}(0)\right)}{P(0),(\forall x)(P(x) \rightarrow P(s(x))),(\forall x)(P(x) \rightarrow P(s(x))) \vdash P\left(s^{3}(0)\right)} \forall: l
\end{aligned}
$$

Its corresponding sets of used auxiliary occurrences are:

$$
\mathcal{A}\left(Q_{(A .1,2)}(\varphi)\right)=\left\{\begin{array}{l}
P(0) \rightarrow P(s(0)) \\
P(s(0)) \rightarrow P\left(s^{2}(0)\right), \\
P\left(s^{2}(0)\right) \rightarrow P\left(s^{3}(0)\right)
\end{array}\right\}
$$

The array-formula formed from these used occurrences is:

$$
Q_{(A .1,2)}^{A}(\varphi)=\left\langle P(0) \rightarrow P(s(0)), P(s(0)) \rightarrow P\left(s^{2}(0)\right), P\left(s^{2}(0)\right) \rightarrow P\left(s^{3}(0)\right)\right\rangle
$$

We can then construct the corresponding substitution of sub-formulas in sequents:

$$
\begin{aligned}
\sigma_{(A .1,2)}^{f s} & =\left\{(A .1,2) / Q_{(A .1,2)}^{A}(\varphi)\right\} \\
& =\left\{(A .1,2) /\left\langle\begin{array}{c}
P(0) \rightarrow P(s(0)) \\
P(s(0)) \rightarrow P\left(s^{2}(0)\right), \\
P\left(s^{2}(0)\right) \rightarrow P\left(s^{3}(0)\right)
\end{array}\right\rangle\right\}
\end{aligned}
$$

Finally, we can extract the Herbrand sequent of the proof:

$$
\begin{aligned}
\left.H_{A}(\varphi)\right)= & \operatorname{set}\left(\mathcal{D}\left(\Phi\left(\overline{\left.s \sigma_{(A .1,2)}^{f s}\right)}\right)\right)\right. \\
= & \operatorname{set}\left(P\left(\Phi\left(P(0) \wedge\left\langle\begin{array}{c}
P(0) \rightarrow P(s(0)), \\
P(s(0)) \rightarrow P\left(s^{2}(0)\right), \\
P\left(s^{2}(0)\right) \rightarrow P\left(s^{3}(0)\right)
\end{array}\right) \vdash P\left(s^{3}(0)\right)\right)\right)\right) \\
= & \operatorname{set}\left(\mathcal{D}\left(\left(\begin{array}{c}
P(0) \wedge P(0) \rightarrow P(s(0)), \\
P(0) \wedge P(s(0)) \rightarrow P\left(s^{2}(0)\right), \\
P(0) \wedge P\left(s^{2}(0)\right) \rightarrow P\left(s^{3}(0)\right)
\end{array}\right) \vdash P\left(s^{3}(0)\right)\right)\right) \\
= & \operatorname{set}\left(\left(\begin{array}{c}
P(0) \wedge P(0) \rightarrow P(s(0)), \\
P(0) \wedge P(s(0)) \rightarrow P\left(s^{2}(0)\right), \\
P(0) \wedge P\left(s^{2}(0)\right) \rightarrow P\left(s^{3}(0)\right)
\end{array}\right) \vdash P\left(s^{3}(0)\right)\right) \\
= & \left(\begin{array}{c}
P(0) \wedge P(0) \rightarrow P(s(0)), \\
P(0) \wedge P(s(0)) \rightarrow P\left(s^{2}(0)\right), \\
P(0) \wedge P\left(s^{2}(0)\right) \rightarrow P\left(s^{3}(0)\right)
\end{array}\right) \vdash P\left(s^{3}(0)\right)
\end{aligned}
$$

Example 4.17 (Extraction of Herbrand Sequent without Proof Transformation and with Nested Quantifiers). Let $\varphi$ be the proof below: 


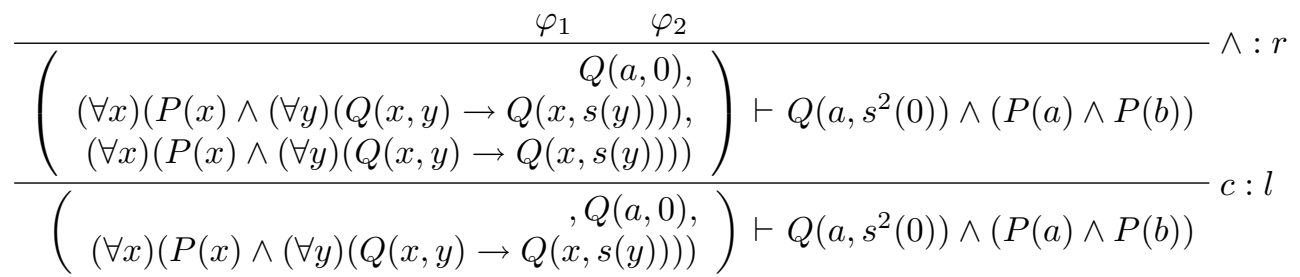

where $\varphi_{1}$ is:

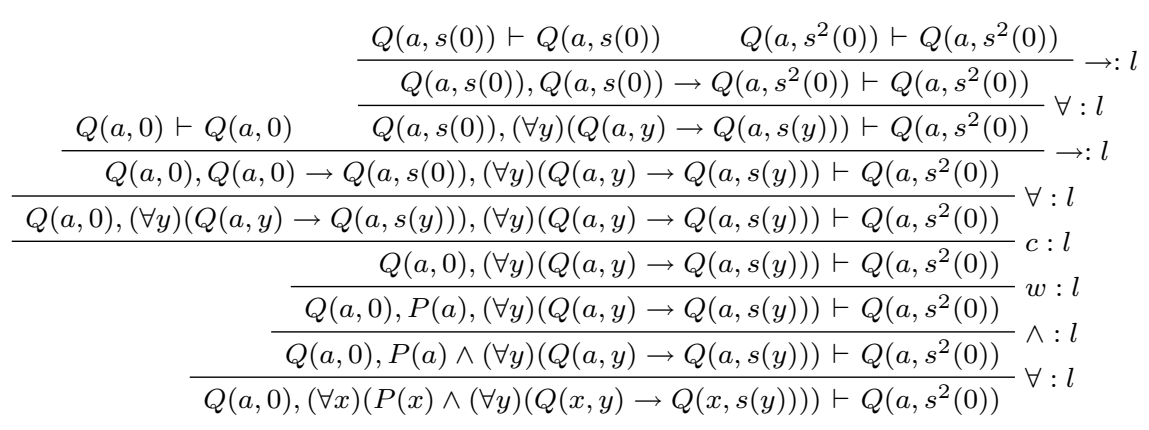

and $\varphi_{2}$ is:

$$
\frac{\left[\varphi_{21}\right] \quad\left[\varphi_{22}\right]}{\frac{\left(\begin{array}{c}
(\forall x)(P(x) \wedge(\forall y)(Q(x, y) \rightarrow Q(x, s(y)))), \\
(\forall x)(P(x) \wedge(\forall y)(Q(x, y) \rightarrow Q(x, s(y))))
\end{array}\right) \vdash P(a) \wedge P(b)}{(\forall x)(P(x) \wedge(\forall y)(Q(x, y) \rightarrow Q(x, s(y)))) \vdash P(a) \wedge P(b)} c: l}
$$

with $\varphi_{21}$ :

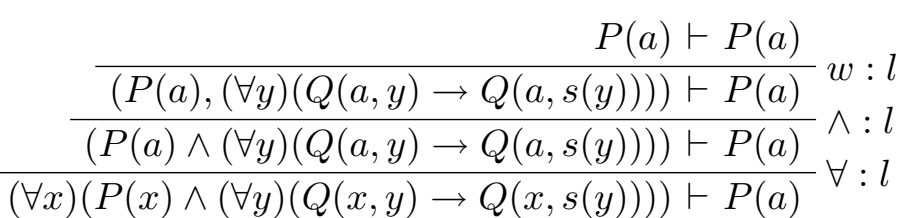

and $\varphi_{22}$ :

$$
\begin{array}{r}
\frac{P(b) \vdash P(b)}{(P(b),(\forall y)(Q(b, y) \rightarrow Q(b, s(y)))) \vdash P(b)} w: l \\
\frac{(P(b) \wedge(\forall y)(Q(b, y) \rightarrow Q(b, s(y)))) \vdash P(b)}{(\forall x)(P(x) \wedge(\forall y)(Q(x, y) \rightarrow Q(x, s(y)))) \vdash P(b)} \forall: l
\end{array}
$$

Firstly we compute the set of positions corresponding to sub-formulas beginning with quantifiers in the end-sequent:

$$
S_{Q S F}(\varphi)=\{(A .2, \varepsilon),(A .2,12)\}
$$

Then we compute the sets of quantifier rules referring to the sub-formulas beginning with quantifiers. In the case of this example, there are two such sets (because the cardinality of $S_{Q S F}$ is 2). The first set, $Q_{(A .2, \varepsilon)}(\varphi)$, has the following rules: 


$$
\begin{gathered}
\frac{Q(a, 0), P(a) \wedge(\forall y)(Q(a, y) \rightarrow Q(a, s(y))) \vdash Q\left(a, s^{2}(0)\right)}{Q(a, 0),(\forall x)(P(x) \wedge(\forall y)(Q(x, y) \rightarrow Q(x, s(y)))) \vdash Q\left(a, s^{2}(0)\right)} \forall: l \\
\frac{(P(a) \wedge(\forall y)(Q(a, y) \rightarrow Q(a, s(y)))) \vdash P(a)}{(\forall x)(P(x) \wedge(\forall y)(Q(x, y) \rightarrow Q(x, s(y)))) \vdash P(a)} \forall: l \\
\frac{(P(b) \wedge(\forall y)(Q(b, y) \rightarrow Q(b, s(y)))) \vdash P(b)}{(\forall x)(P(x) \wedge(\forall y)(Q(x, y) \rightarrow Q(x, s(y))) \vdash P(a)} \forall: l
\end{gathered}
$$

The second set, $Q_{(A .2,12)}$, has the following rules:

$$
\begin{aligned}
& \frac{Q(a, s(0)), Q(a, s(0)) \rightarrow Q\left(a, s^{2}(0)\right) \vdash Q\left(a, s^{2}(0)\right)}{Q(a, s(0)),(\forall y)(Q(a, y) \rightarrow Q(a, s(y))) \vdash Q\left(a, s^{2}(0)\right)} \forall: l \\
& \frac{\left(\begin{array}{r}
Q(a, 0), \\
Q(a, 0) \rightarrow Q(a, s(0)), \\
(\forall y)(Q(a, y) \rightarrow Q(a, s(y)))
\end{array}\right) \vdash Q\left(a, s^{2}(0)\right)}{Q(a, 0),}, \forall: l
\end{aligned}
$$

The sets of used auxiliary occurrences are:

$$
\mathcal{A}\left(Q_{(A .2, \varepsilon)}(\varphi)\right)=\left\{\begin{array}{l}
P(a) \wedge(\forall y)(Q(a, y) \rightarrow Q(a, s(y))), \\
P(b) \wedge(\forall y)(Q(b, y) \rightarrow Q(b, s(y))))
\end{array}\right\}
$$

and:

$$
\mathcal{A}\left(Q_{(A .2,12)}(\varphi)\right)=\left\{Q(a, s(0)) \rightarrow Q\left(a, s^{2}(0)\right), Q(a, 0) \rightarrow Q(a, s(0))\right\}
$$

The array-formulas formed from these used occurrences are:

$$
\begin{gathered}
Q_{(A .2, \varepsilon)}^{A}(\varphi)=\left\langle\begin{array}{rl}
P(a) & \wedge(\forall y)(Q(a, y) \rightarrow Q(a, s(y))), \\
P(b) & \wedge(\forall y)(Q(b, y) \rightarrow Q(b, s(y)))
\end{array}\right\rangle \\
Q_{(A .2,12)}^{A}(\varphi)=\left\langle Q(a, s(0)) \rightarrow Q\left(a, s^{2}(0)\right), Q(a, 0) \rightarrow Q(a, s(0))\right\rangle
\end{gathered}
$$

We can then construct the corresponding 2 substitutions of sub-formulas in sequents:

$$
\begin{aligned}
\sigma_{(A .2, \varepsilon)}^{f s} & =\left\{(A .2, \varepsilon) / Q_{(A .2, \varepsilon)}^{A}(\varphi)\right\} \\
& =\left\{(A .2, \varepsilon) /\left\langle\begin{array}{c}
P(a) \wedge(\forall y)(Q(a, y) \rightarrow Q(a, s(y))), \\
P(b) \wedge(\forall y)(Q(b, y) \rightarrow Q(b, s(y))))
\end{array}\right\rangle\right\} \\
\sigma_{(A .2,12)}^{f s} & =\left\{(A .2,12) / Q_{(A .2,12)}^{A}(\varphi)\right\} \\
& =\left\{(A .2,12) /\left\langle Q(a, s(0)) \rightarrow Q\left(a, s^{2}(0)\right), Q(a, 0) \rightarrow Q(a, s(0))\right\rangle\right\}
\end{aligned}
$$


Finally, we can extract the Herbrand sequent of the proof (explanations on each of the derivation steps are given subsequently):

$$
\begin{aligned}
& H_{A}(\varphi)={ }^{1} \operatorname{set}\left(P\left(\Phi\left(\overline{\left.s\left(\sigma_{(A .2, \varepsilon)}^{f s} \circ \sigma_{(A .2,12)}^{f s}\right)\right)}\right)\right)\right. \\
& =^{2} \operatorname{set}(\mathcal{D}(\Phi((Q(a, 0), \\
& (\forall x)(P(x) \wedge(\forall y)(Q(x, y) \rightarrow Q(x, s(y)))) \\
& \left.\left.\left.\left.\vdash Q\left(a, s^{2}(0)\right) \wedge(P(a) \wedge P(b))\right) \overline{\left(\sigma_{(A .2, \varepsilon)}^{f s} \circ \sigma_{(A .2,12)}^{f s}\right)}\right)\right)\right) \\
& =^{3} \operatorname{set}(\mathcal{D}(\Phi)((Q(a, 0), \\
& (\forall x)(P(x) \wedge(\forall y)(Q(x, y) \rightarrow Q(x, s(y)))) \\
& \left.\left.\left.\left.\vdash Q\left(a, s^{2}(0)\right) \wedge(P(a) \wedge P(b))\right) \overline{\sigma_{(A .2, \varepsilon)}^{f s}} \overline{\sigma_{(A .2,12)}^{f s}}\right)\right)\right) \\
& =^{4} \operatorname{set}(\mathcal{D}(\Phi((Q(a, 0), \\
& \langle P(a) \wedge(\forall y)(Q(a, y) \rightarrow Q(a, s(y))), \\
& P(b) \wedge(\forall y)(Q(b, y) \rightarrow Q(b, s(y))))\rangle \\
& \left.\left.\left.\left.\vdash Q\left(a, s^{2}(0)\right) \wedge(P(a) \wedge P(b))\right) \overline{\sigma_{(A .2,12)}^{f s}}\right)\right)\right) \\
& =^{5} \operatorname{set}(\mathcal{D}(\Phi((Q(a, 0), \\
& \left\langleP ( a ) \wedge \left\langle Q(a, s(0)) \rightarrow Q\left(a, s^{2}(0)\right),\right.\right. \\
& Q(a, 0) \rightarrow Q(a, s(0))\rangle, \\
& P(b) \wedge\langle(\forall y)(Q(b, y) \rightarrow Q(b, s(y))\rangle\rangle \\
& \left.\left.\left.\left.\vdash Q\left(a, s^{2}(0)\right) \wedge(P(a) \wedge P(b))\right)\right)\right)\right) \\
& ={ }^{6} \operatorname{set}(\mathcal{D}(\Phi((Q(a, 0) \text {, } \\
& P(a) \wedge\left\langle Q(a, s(0)) \rightarrow Q\left(a, s^{2}(0)\right),\right. \\
& Q(a, 0) \rightarrow Q(a, s(0))\rangle, \\
& P(b) \wedge\langle(\forall y)(Q(b, y) \rightarrow Q(b, s(y))\rangle \\
& \left.\left.\left.\left.\vdash Q\left(a, s^{2}(0)\right) \wedge(P(a) \wedge P(b))\right)\right)\right)\right) \\
& ={ }^{7} \operatorname{set}(\mathcal{D}(\Phi((Q(a, 0) \text {, } \\
& P(a) \wedge\left(Q(a, s(0)) \rightarrow Q\left(a, s^{2}(0)\right)\right), \\
& P(a) \wedge(Q(a, 0) \rightarrow Q(a, s(0))), \\
& P(b) \wedge\langle(\forall y)(Q(b, y) \rightarrow Q(b, s(y))\rangle \\
& \left.\left.\left.\left.\vdash Q\left(a, s^{2}(0)\right) \wedge(P(a) \wedge P(b))\right)\right)\right)\right) \\
& ={ }^{8} \operatorname{set}(\mathcal{D}((Q(a, 0) \text {, } \\
& P(a) \wedge\left(Q(a, s(0)) \rightarrow Q\left(a, s^{2}(0)\right)\right), \\
& P(a) \wedge(Q(a, 0) \rightarrow Q(a, s(0))), \\
& P(b) \wedge(\forall y)(Q(b, y) \rightarrow Q(b, s(y)) \\
& \left.\left.\left.\vdash Q\left(a, s^{2}(0)\right) \wedge(P(a) \wedge P(b))\right)\right)\right)
\end{aligned}
$$




$$
\begin{aligned}
=9 \quad & \operatorname{set}((Q(a, 0), \\
P(a) & \wedge\left(Q(a, s(0)) \rightarrow Q\left(a, s^{2}(0)\right)\right), \\
P(a) & \wedge(Q(a, 0) \rightarrow Q(a, s(0))), \\
P(b) & \wedge\left(\left(Q\left(b, c^{*}\right) \rightarrow Q\left(b, s\left(c^{*}\right)\right)\right.\right. \\
\vdash & \left.\left.Q\left(a, s^{2}(0)\right) \wedge(P(a) \wedge P(b))\right)\right) \\
=^{10} \quad Q(a, 0) & \\
P(a) & \wedge\left(Q(a, s(0)) \rightarrow Q\left(a, s^{2}(0)\right)\right), \\
P(a) & \wedge(Q(a, 0) \rightarrow Q(a, s(0))), \\
& P(b) \wedge\left(Q\left(b, c^{*}\right) \rightarrow Q\left(b, s\left(c^{*}\right)\right)\right) \\
\vdash & \left.Q\left(a, s^{2}(0)\right) \wedge(P(a) \wedge P(b))\right)
\end{aligned}
$$

Explanations for each derivation step:

1. Expansion of the definition of $H_{A}(\varphi)$.

2. Expansion of the end-sequent $s$.

3. Expansion of the definition of the composition of substitutions.

4. Application of the unifying substitution $\overline{\sigma_{(A .2, \varepsilon)}^{f s}}$.

Since $(P(x) \wedge(\forall y)(Q(x, y) \rightarrow Q(x, s(y))))$ unifies with the formulas in $Q_{(A, 2, \varepsilon)}^{A}(\varphi)((P(a) \wedge(\forall y)(Q(a, y) \rightarrow Q(a, s(y))))$ and $(P(b) \wedge(\forall y)(Q(b, y) \rightarrow$ $Q(b, s(y)))))$, the occurrence $(\forall x)(P(x) \wedge(\forall y)(Q(x, y) \rightarrow Q(x, s(y))))$ is substituted by $Q_{(A, 2, \varepsilon)}^{A}(\varphi)$.

5. Application of the unifying substitution $\overline{\sigma_{(A .2,12)}^{f s}}$. Since $(Q(a, y) \rightarrow$ $Q(a, s(y)))$ unifies with with the formulas in $Q_{(A .2,12)}^{A}(\varphi)(Q(a, s(0)) \rightarrow$ $Q\left(a, s^{2}(0)\right)$ and $\left.Q(a, 0) \rightarrow Q(a, s(0))\right),(\forall y)(Q(a, y) \rightarrow Q(a, s(y)))$ is substituted by $Q_{(A .2,12)}^{A}(\varphi)$. On the other hand $(Q(b, y) \rightarrow Q(b, s(y)))$, which is also in position $(A .2,12)$, does not unify with the formulas in $Q_{(A .2,12)}^{A}(\varphi)$, because the term $b$ clashes with the term a. Therefore $(\forall y)(Q(b, y) \rightarrow Q(b, s(y)))$ remains unchanged (not substituted). This exemplifies the importance of performing a unifying substitution instead of just a normal substitution. Different branches of the tree required the quantified $x$ to be instantiated to $b$ and to $a$. Then only the branch of the tree responsible for the instantiation of $x$ to a required the quantified $y$ to be instantiated, according to the formulas in $Q_{(A .2,12)}^{A}(\varphi)$. If we had performed a normal substitution, we would have undesirably erased the instantiation of $x$ to $b$ due to the other branch of the tree. Unification is thus used to take care of branching and contractions in the LK-Proof. 
6. First iteration in the expansion of array-formulas, by way of $\Phi$.

7. Second iteration in the expansion of array-formulas, by way of $\Phi$.

8. Third iteration in the expansion of array-formulas, by way of $\Phi$.

9. Dequantification of the sequent. Since $(\forall y)(Q(b, y) \rightarrow Q(b, s(y)))$ is not used (it appeared only by weakenings in $\varphi$ ), it was not substituted by any unifying substitution. Hence an undesired quantifier remained in the sequent. The dequantification simply removes the quantifier and substitutes $y$ by an eigen-constant $c *$, without affecting the validity of the sequent, since the fact that it appeared by weakening means that this sub-formula is actually irrelevant for the proof. We could have simply removed the sub-formula completely, but the definition of Herbrand sequent requires instantiations of the complete formulas.

10. Set-normalization of the sequent.

Example 4.18 (Constructing a Proof for $H_{A}(\varphi)$ ). Let $\varphi$ be the LK-Proof below:

$$
\frac{\left[\varphi^{0}\right] \quad\left[\varphi^{1}\right]}{\frac{(P(0) \wedge(\forall y)(Q(y))) \wedge(\forall x)(P(x)) \vdash P(0) \wedge P(1)}{(P(0) \wedge(\forall y)(Q(y))) \wedge(\forall x)(P(x)) \vdash(P(0) \wedge P(1)) \wedge P(2)}}
$$

where $\varphi^{0}$ is:

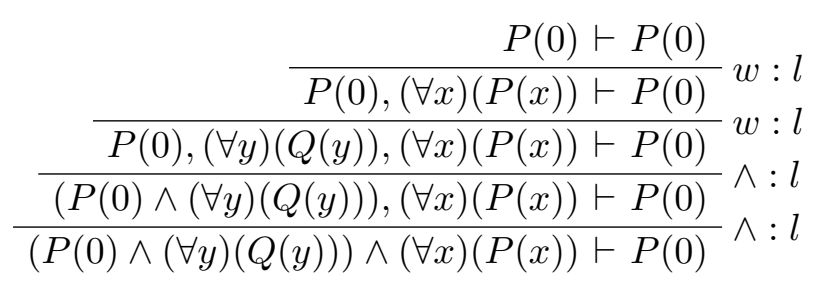

and $\varphi^{1}$ is:

$$
\begin{aligned}
& \frac{P(1) \vdash P(1)}{(\forall x) P(x) \vdash P(1)} \forall: l \\
& \frac{(\forall x) P(x) \vdash P(1)}{P(0),(\forall x) P(x) \vdash P(1)} w: l \\
& \overline{P(0),(\forall y) Q(y),(\forall x) P(x) \vdash P(1)} w: l \\
& \frac{\frac{(P(0) \wedge(\forall y) Q(y)),(\forall x) P(x) \vdash P(1)}{(P(0) \wedge(\forall y) Q(y)) \wedge(\forall x) P(x) \vdash P(1)} \wedge: l}{} \wedge l
\end{aligned}
$$

and $\varphi^{2}$ is: 


$$
\begin{aligned}
& \frac{P(2) \vdash P(2)}{(\forall x) P(x) \vdash P(2)} \forall: l \\
& \frac{(\forall),(\forall x) P(x) \vdash P(2)}{P(x) P}, l \\
& \overline{P(0),(\forall y) Q(y),(\forall x) P(x) \vdash P(2)} w: l \\
& \frac{\frac{P(P) \wedge(\forall y) Q(y)),(\forall x) P(x) \vdash P(2)}{(P(0) \wedge(\forall y) Q(y)) \wedge(\forall x) P(x) \vdash P(2)} \wedge: l}{\wedge l}
\end{aligned}
$$

Its Herbrand sequent, extracted by algorithm 4.6 is:

$$
H_{A}(\varphi)=\left(P(0) \wedge\left(Q\left(c_{1}^{*}\right)\right)\right) \wedge P(1),\left(P(0) \wedge Q\left(c_{2}^{*}\right)\right) \wedge P(2) \vdash(P(0) \wedge P(1)) \wedge P(2)
$$

\begin{tabular}{|c|c|}
\hline$\left[\varphi_{1}^{0}\right] \quad\left[\varphi_{1}^{1}\right]$ & \\
\hline$(P(0) \wedge(\forall y)(Q(y))) \wedge(\forall x)(P(x)) \vdash P(0) \wedge P(1)$ & {$\left[\varphi_{1}^{2}\right]$} \\
\hline
\end{tabular}

Now we transform $\varphi$ into an $\mathbf{L K}$-Proof $\varphi^{*}$ of this Herbrand Sequent. Firstly, we perform the unifying substitutions in the inductive way explained in Lemma 4.9, and we obtain the pseudo-proof $\varphi_{1}$ below, where false inference rules are marked with $*$ :

where $\varphi_{1}^{0}$ is:

$$
\begin{array}{r}
\frac{P(0) \vdash P(0)}{P(0), P(1), P(2) \vdash P(0)} w: l \\
\frac{P(0),(\forall y)(Q(y)), P(1), P(2) \vdash P(0}{\frac{(P(0) \wedge(\forall y)(Q(y))), P(1), P(2) \vdash P(0)}{P(1),(P(0) \wedge(\forall y)(Q(y)) \wedge P(2) \vdash P(0)} \wedge} \wedge: l \\
\wedge l^{*}
\end{array}
$$

and $\varphi_{1}^{1}$ is:

$$
\begin{array}{r}
\frac{P(1) \vdash P(1)}{P(1), P(2) \vdash P(1)} \\
\frac{P(0), P(1), P(2) \vdash P(1)}{P} w l \\
\frac{\frac{P(0),(\forall y)(Q(y)), P(1), P(2) \vdash P(1)}{(P(0) \wedge(\forall y)(Q(y))), P(1), P(2) \vdash P(1)}}{(P(0) \wedge(\forall y)(Q(y))) \wedge P(1),(P(0) \wedge(\forall y)(Q(y))) \wedge P(2) \vdash P(1)} \wedge: l \\
\end{array}
$$

and $\varphi_{1}^{2}$ is:

$$
\begin{aligned}
& \frac{P(2) \vdash P(2)}{P(1), P(2) \vdash P(2)} \forall: l
\end{aligned}
$$

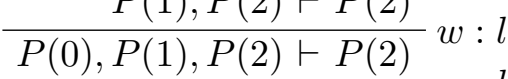

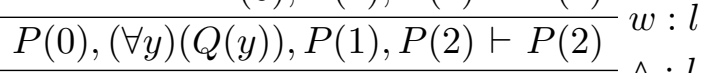

$$
\begin{aligned}
& \overline{(P(0) \wedge(\forall y)(Q(y))), P(1), P(2) \vdash P(2)} \wedge: l \\
& \overline{(P(0) \wedge(\forall y)(Q(y))) \wedge P(1),(P(0) \wedge(\forall y)(Q(y))) \wedge P(2) \vdash P(2)} \wedge: l^{*}
\end{aligned}
$$


Then we perform dequantification and we obtain $\varphi_{2}$ below:

\begin{tabular}{|c|c|}
\hline$\left[\varphi_{2}^{0}\right] \quad\left[\varphi_{2}^{1}\right]$ & \\
\hline$\left(P(0) \wedge Q\left(c_{1}^{*}\right)\right) \wedge P(1),\left(P(0) \wedge Q\left(c_{2}^{*}\right)\right) \wedge P(2) \vdash P(0) \wedge P(1)$ & {$\left[\varphi_{2}^{2}\right]$} \\
\hline$\left(P(0) \wedge Q\left(c_{1}^{*}\right)\right) \wedge P(1),\left(P(0) \wedge Q\left(c_{2}^{*}\right)\right) \wedge P(2) \vdash(P(0) \wedge P(1)$ & \\
\hline
\end{tabular}

where $\varphi_{2}^{0}$ is:

$$
\begin{aligned}
& \frac{P(0) \vdash P(0)}{P(0), P(1), P(2) \vdash P(0)} w: l \\
& \frac{\frac{P(0), Q\left(c_{1}^{*}\right), P(1), P(2) \vdash P(0)}{\left(P(0) \wedge Q\left(c_{1}^{*}\right)\right), P(1), P(2) \vdash P(0)} \wedge: l}{\wedge} \wedge_{l}
\end{aligned}
$$

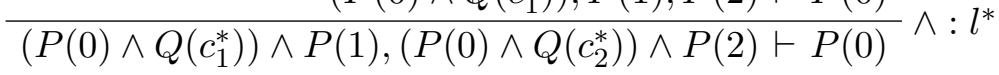

and $\varphi_{2}^{1}$ is:

$$
\begin{array}{r}
\frac{P(1) \vdash P(1)}{P(1), P(2) \vdash P(1)} \\
\frac{P(0), P(1), P(2) \vdash P(1)}{P} w: l \\
\frac{\frac{P(0), Q\left(c_{1}^{*}\right), P(1), P(2) \vdash P(1)}{\left(P(0) \wedge Q\left(c_{1}^{*}\right)\right), P(1), P(2) \vdash P(1)}}{\left(P(0) \wedge Q\left(c_{1}^{*}\right)\right) \wedge P(1),\left(P(0) \wedge Q\left(c_{2}^{*}\right)\right) \wedge P(2) \vdash P(1)} \wedge: l \\
\wedge: l^{*}
\end{array}
$$

and $\varphi_{2}^{2}$ is:

$$
\begin{aligned}
& \frac{P(2) \vdash P(2)}{P(1), P(2) \vdash P(2)} \forall: l \\
& \frac{P(0), P(1), P(2) \vdash P(2)}{P(0), Q\left(c_{2}^{*}\right), P(1), P(2) \vdash P(2)} w: l
\end{aligned}
$$

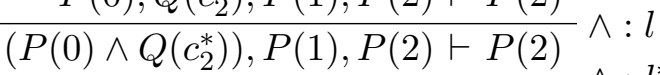

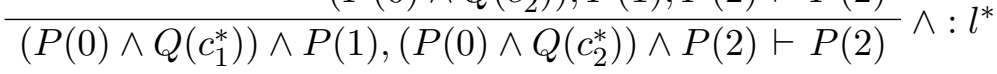

Finally, we insert the missing weakenings and propositional-rules, in order to obtain the desired LK-Proof $\varphi^{*}$ :

$$
\frac{\left[\varphi_{0}^{*}\right]\left[\varphi_{1}^{*}\right]}{\frac{\left(P(0) \wedge Q\left(c_{1}^{*}\right)\right) \wedge P(1),\left(P(0) \wedge Q\left(c_{2}^{*}\right)\right) \wedge P(2) \vdash P(0) \wedge P(1)}{\left(P(0) \wedge Q\left(c_{1}^{*}\right)\right) \wedge P(1),\left(P(0) \wedge Q\left(c_{2}^{*}\right)\right) \wedge P(2) \vdash(P(0) \wedge P(1)) \wedge P(2)} \quad\left[\varphi_{2}^{*}\right]}
$$

where $\varphi_{0}^{*}$ is: 


$$
\begin{aligned}
& P(0) \vdash P(0) \\
& \frac{P(0) \vdash P(0)}{P(0), P(1) \vdash P(0)} w: l \\
& \overline{P(0), P(1), P(2) \vdash P(0)} w: l
\end{aligned}
$$

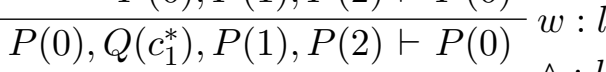

$$
\begin{aligned}
& \overline{\left(P(0) \wedge Q\left(c_{1}^{*}\right)\right), P(1), P(2) \vdash P(0)} \wedge: l
\end{aligned}
$$

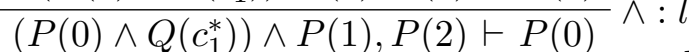

$$
\begin{aligned}
& \frac{\overline{\left(P(0) \wedge Q\left(c_{1}^{*}\right)\right) \wedge P(1),\left(P(0) \wedge Q\left(c_{2}^{*}\right)\right), P(2) \vdash P(0)}}{\left(P(0) \wedge Q\left(c_{1}^{*}\right)\right) \wedge P(1),\left(P(0) \wedge Q\left(c_{2}^{*}\right)\right) \wedge P(2) \vdash P(0)} \wedge: l
\end{aligned}
$$

and $\varphi_{1}^{*}$ is:

$$
\begin{aligned}
& \frac{\frac{P(1) \vdash P(1)}{P(1), P(2) \vdash P(1)} w: l}{P(0), P(1), P(2) \vdash P(1)} w: l \\
& \frac{P(0), P(1), P(2) \vdash P(1)}{P(0), Q\left(c_{1}^{*}\right), P(1), P(2) \vdash P(1)} w: l \\
& \left(P(0) \wedge Q\left(c_{1}^{*}\right)\right), P(1), P(2) \vdash P(1) \wedge: l
\end{aligned}
$$

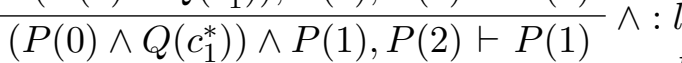

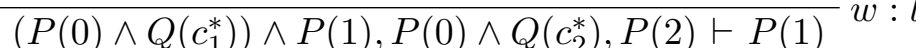

$$
\begin{aligned}
& \overline{\left(P(0) \wedge Q\left(c_{1}^{*}\right)\right) \wedge P(1),\left(P(0) \wedge Q\left(c_{2}^{*}\right)\right) \wedge P(2) \vdash P(1)} \wedge: l
\end{aligned}
$$

and $\varphi_{2}^{*}$ is:

$$
\begin{aligned}
& P(2) \vdash P(2) \\
& P(1), P(2) \vdash P(2) \quad \forall:
\end{aligned}
$$

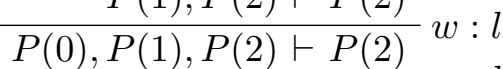

$$
\begin{aligned}
& \overline{P(0), Q\left(c_{2}^{*}\right), P(1), P(2) \vdash P(2)} w: l
\end{aligned}
$$

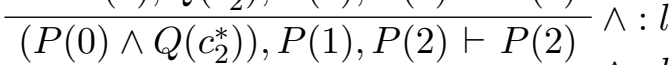

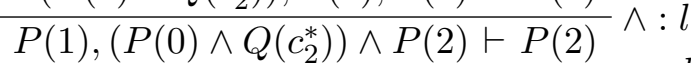

$$
\begin{aligned}
& \frac{\overline{\left(P(0) \wedge Q\left(c_{1}^{*}\right)\right), P(1),\left(P(0) \wedge Q\left(c_{2}^{*}\right)\right) \wedge P(2) \vdash P(2)}}{\left(P(0) \wedge Q\left(c_{1}^{*}\right)\right) \wedge P(1),\left(P(0) \wedge Q\left(c_{2}^{*}\right)\right) \wedge P(2) \vdash P(2)} \wedge: l
\end{aligned}
$$




\section{Chapter 5}

\section{Conclusion}

The main contribution of this thesis is the development of an algorithm for the extraction of Herbrand sequents from formal proofs written in the Sequent Calculus LK. This algorithm draws ideas from two previously existing algorithms in a way that combines their strengths but avoids their weaknesses. Namely, the algorithm here developed also accepts proofs in non-prenex form, and it doesn't need to perform expensive proof transformations to obtain the desired Herbrand sequent.

The resulting Herbrand sequent acts as a summarization of the interesting content of the formal proof, thus improving its human-understandability. However, since the algorithm is restricted to the Sequent Calculus LK for First-Order Logic, it remains for future work to investigate how it could be adapted and extended to other calculi and other logics. 


\section{Bibliography}

[1] M.Baaz; A.Leitsch. On skolemization and proof complexity. Fundamenta Matematicae, (20):353-379, 1994.

[2] Matthias Baaz, Stefan Hetzl, Alexander Leitsch, Clemens Richter, and Hendrik Spohr. Ceres: An Analysis of Fürstenberg's Proof of the Infinity of Primes. in preparation.

[3] Matthias Baaz, Stefan Hetzl, Alexander Leitsch, Clemens Richter, and Hendrik Spohr. Cut-Elimination: Experiments with CERES. In Franz Baader and Andrei Voronkov, editors, Logic for Programming, Artificial Intelligence, and Reasoning (LPAR) 2004, volume 3452 of Lecture Notes in Computer Science, pages 481-495. Springer, 2005.

[4] Matthias Baaz, Stefan Hetzl, Alexander Leitsch, Clemens Richter, and Hendrik Spohr. Proof Transformation by CERES. In Jonathan M. Borwein and William M. Farmer, editors, Mathematical Knowledge Management (MKM) 2006, volume 4108 of Lecture Notes in Artificial Intelligence, pages 82-93. Springer, 2006.

[5] S. R. Buss. On Herbrand's theorem. Lecture Notes in Computer Science, 960:195, 1995.

[6] G. Gentzen. Untersuchungen über das logische schließen. In M.E.Szabo, editor, The Collected Papers of Gerhard Gentzen, pages 68-131. NorthHolland Publishing Company, Amsterdam - London, 1969.

[7] Stefan Hetzl. The two dual parts of formal proofs. In preparation.

[8] Stefan Hetzl. Projection-based cut-elimination and normalization. Diplom, Technische Universitt Wien, Wien, March 2004.

[9] J.Herbrand. Recherches sur la Theorie de la Demonstration. PhD thesis, University of Paris, 1930.

[10] M. Baaz; A. Leitsch. Cut normal forms and proof complexity. Annals of Pure and Applied Logic, 97:127-177, 1999. 
[11] G. Sutcliffe and C. Suttner. The State of CASC. AI Communications, 19(1):35-48, 2006. 